\title{
Ground-Water-Level Monitoring, Basin Boundaries, and Potentiometric Surfaces of the Aquifer System at Edwards Air Force Base, California, 1992
}

By DIANE L. REWIS

U.S. GEOLOGICAL SURVEY

Water-Resources Investigations Report 95-4131

Prepared in cooperation with the U.S. DEPARTMENT OF THE AIR FORCE, EDWARDS AIR FORCE BASE, AIR FORCE FLIGHT TEST CENTER

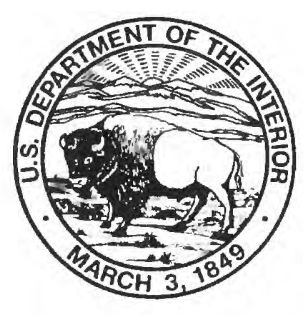




\section{U.S. DEPARTMENT OF THE INTERIOR \\ BRUCE BABBITT, Secretary}

U.S. GEOLOGICAL SURVEY

Gordon P. Eaton, Director

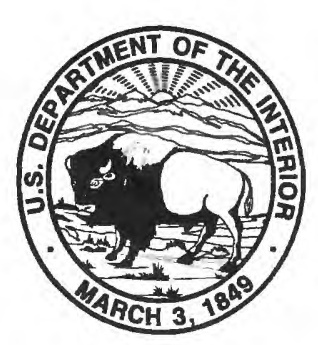

The use of firm, trade, and brand names in this report is for identification purposes only and does not constitute endorsement by the U.S. Geological Survey.

For addtional information write to:

Copies of this report can be purchased from:

District Chief

U.S. Geological Survey

Federal Building, Room W-2233

2800 Cottage Way

Sacramento, CA 95825
U.S. Geological Survey

Earth Science Information Center

Open-File Report Section

Box 25286, Mail Stop 417

Denver Federal Center

Denver, CO 80225 


\section{CONTENTS}

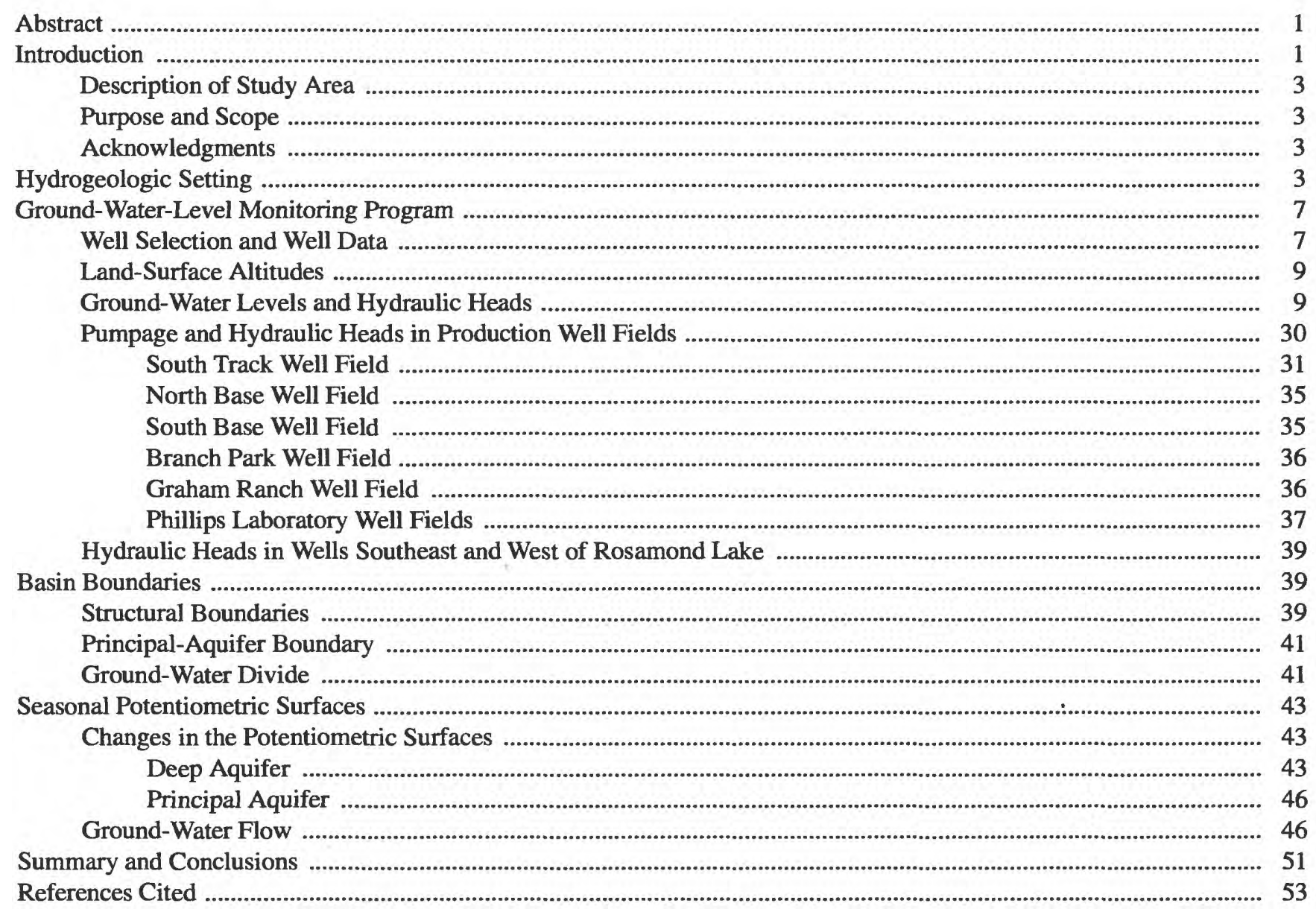

\section{FIGURES}

1-3. Maps showing:

1. Location of study area.

2. General geology and location of ground-water subbasins in the Antelope Valley, California

3. General geology and ground-water basin boundaries at Edwards Air Force Base, California, and geologic section $A-A^{\prime}$

4. Geologic section $A-A^{\prime}$ showing the principal aquifer boundary and the ground-water divide between the

Lancaster and North Muroc subbasins, Edwards Air Force Base, California

5. Map showing locations of wells and piezometers on Edwards Air Force Base, California..

6-14. Graphs showing:

6. Hydraulic heads for U.S. Geological Survey piezometers on Edwards Air Force Base,

$$
\text { California, } 1992 \text {. }
$$

7. Total monthly pumpage at Edwards Air Force Base, California

8. Monthly pumpage from and hydraulic heads in wells and selected piezometers in and near the South Track well field, Edwards Air Force Base, California

9. Monthly pumpage from and hydraulic heads in wells and selected piezometers in and near the North Base well field, Edwards Air Force Base, California 
10. Monthly pumpage from and hydraulic heads in wells in the South Base well field, Edwards Air Force Base, California

11. Monthly pumpage from and hydraulic heads in wells and selected piezometers in and near the

Branch Park well field, Edwards Air Force Base, California

12. Monthly pumpage from and hydraulic heads in wells and selected piezometers in and near the Graham Ranch well field, Edwards Air Force Base, California .

13. Monthly pumpage from and hydraulic heads in wells and selected piezometers in and near the Phillips Laboratory well fields, Edwards Air Force Base, California

14. Hydraulic heads in wells south and west of Buckhorn and Rosamond Lakes, Edwards Air Force Base, California.

15-19. Maps showing:

15. Potentiometric surface of the deep aquifer, Edwards Air Force Base, California, spring 1992................ 54

16. Potentiometric surface of the deep aquifer, Edwards Air Force Base, California, late summer 1992...... 56

17. Potentiometric surface of the principal aquifer, Edwards Air Force Base, California, spring 1992 .......... 58

18. Potentiometric surface of the principal aquifer, Edwards Air Force Base, California, late summer 1992.

19. Change in hydraulic head in wells and piezometers completed in the deep aquifer, Edwards Air Force Base, California, spring to late summer 1992.

20. Hydraulic-head profiles for geologic sections $B-B^{\prime}, C-C^{\prime}$, and $D-D^{\prime}$, Edwards Air Force Base, California.

\section{TABLES}

1. Well-construction data and historic water-level data for wells and piezometers on and near

Edwards Air Force Base, California.

2. Ground-water levels for wells and piezometers on Edwards Air Force Base, California, 1992

3. Monthly and annual pumpage data from production wells on Edwards Air Force Base, California, 1992 ......... 32

4. Altitudes of confining-unit interval and bedrock-alluvium contacts for wells on and near Edwards Air Force Base, California

5. Hydraulic heads and change in hydraulic heads for selected wells and piezometers used to plot the

potentiometric surfaces of the aquifer system at Edwards Air Force Base, California, $1992 . . . . . . .$.
6. Hydraulic gradients for four subregional ground-water-flow directions in the deep aquifer at Edwards Air Force Base, California, 1992

\section{CONVERSION FACTORS}

\begin{tabular}{rcl}
\hline Multiply & By & To obtain \\
\hline acre-feet (acre-ft) & 1,233 & cubic meter \\
foot (ft) & 0.3048 & meter \\
gallon (gal) & 3.785 & liter \\
inch (in.) & 2.54 & centimeter \\
inch per year (in/yr) & 2.54 & centimeter per year \\
mile (mi) & 1.609 & kilometer \\
square mile (mi & 2.590 & square kilometer \\
\hline
\end{tabular}

In this report, units of measurement for recharge and pumpage are given in acre-feet and gallons. Acre-feet can be converted to gallons by multiplying by $3.259 \times 10^{5}$. Raw data were reported by Edwards Air Force Base in thousand gallons.

\section{Vertical Datum}

Sea level: In this report "sea level" refers to the National Geodetic Vertical Datum of 1929-a geodetic datum derived from a general adjustment of the first-order level nets of the United States and Canada, formerly called Sea Level Datum of 1929.

\section{Acronyms}

EAFB Edwards Air Force Base

GPS Global Positioning System

USGS U.S. Geological Survey 
Wells are identified and numbered according to their location in the rectangular system for subdivision of public lands. For example, in well number 009N010W34R002S, the identification number consists of the township number, north or south; the range number, east or west; and the section number. Each section is further divided into sixteen 40-acre tracts lettered consecutively (except I and $\mathrm{O}$ ), beginning with " $\mathrm{A}$ " in the northeast corner of the section and progressing in a sinusoidal manner to " $\mathrm{R}$ " in the southeast corner. Within each 40 -acre tract, wells are sequentially numbered in the order that they are inventoried. The final letter refers to the base line and meridian. In California, there are three base lines and meridians: Humbolt (H), Mount Diablo (M), and San Bernardino (S). Because all wells in the study area are referenced to the San Bernardino base line and meridian (S), the final letter will be omitted. In this report, well numbers are abbreviated and written 9N/10W-34R2. Wells in the same township and range may be referred to only by their section designation, $-34 \mathrm{R} 2$. The following diagram shows how the number for well 9N/10W-34R2 is derived.

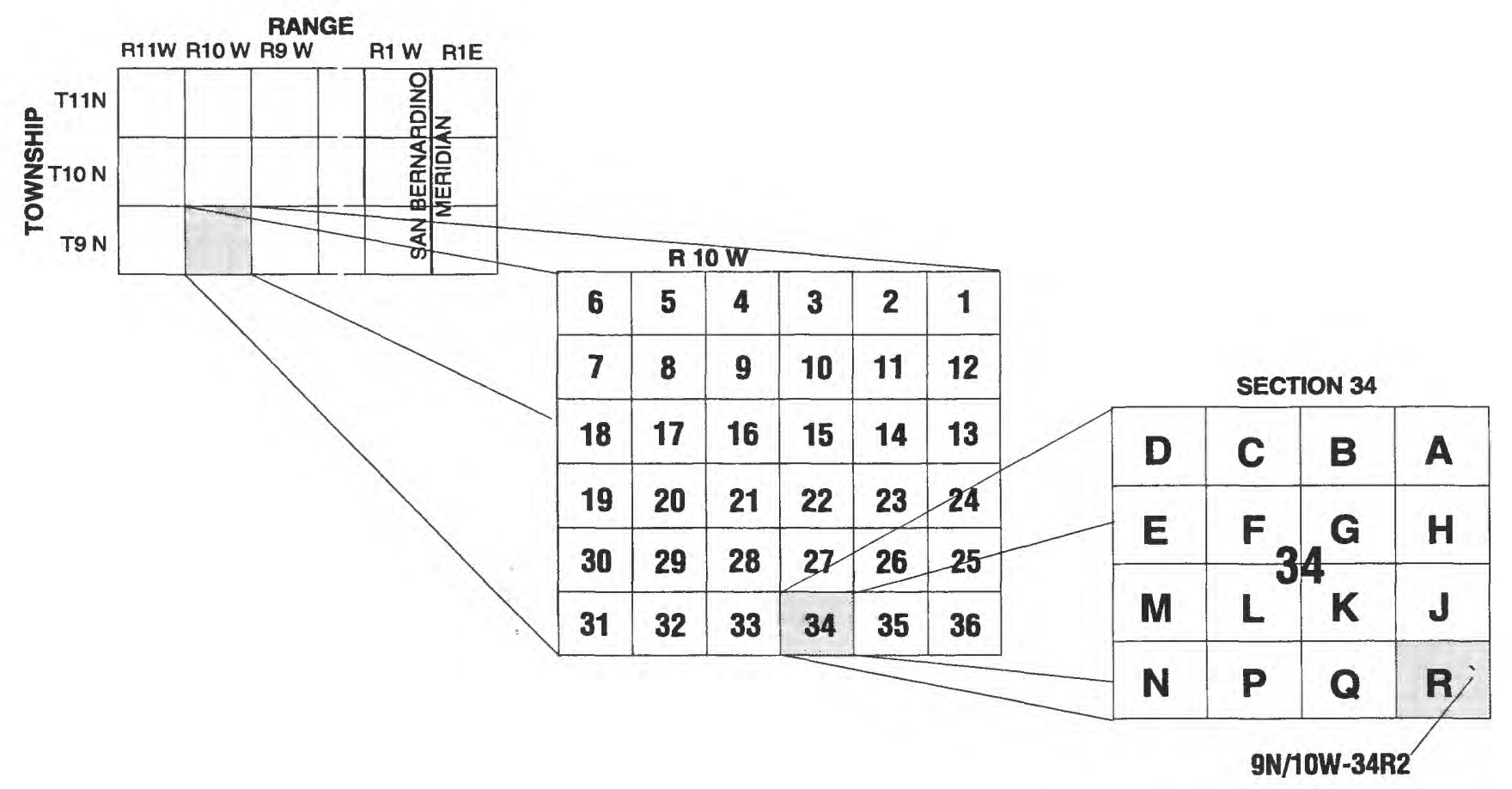




\title{
Ground-Water-Level Monitoring, Basin Boundaries, and Potentiometric Surfaces of the Aquifer System at Edwards Air Force Base, California, 1992
}

\author{
By Diane L. Rewis
}

\section{Abstract}

A ground-water-level monitoring program was implemented at Edwards Air Force Base, California, from January through December 1992 to monitor spatial and temporal changes in potentiometric surfaces that largely are affected by ground-water pumping. Potentiometric-surface maps are needed to determine the correlation between declining ground-water levels and the distribution of land subsidence. The monitoring program focused on areas of the base where pumping has occurred, especially near Rogers Lake, and involved three phases of data collection: (1) well canvassing and selection, (2) geodetic surveys, and (3) monthly ground-water-level measurements. Construction and historical waterlevel data were compiled for 118 wells and piezometers on or near the base, and monthly ground-water-level measurements were made in 82 wells and piezometers on the base.

The compiled water-level data were used in conjunction with previously collected geologic data to identify three types of no-flow boundaries in the aquifer system: structural boundaries, a principal-aquifer boundary, and ground-water divides. Heads were computed from groundwater-level measurements and land-surface altitudes and then were used to map seasonal potentiometric surfaces for the principal and deep aquifers underlying the base. Pumping has created a regional depression in the potentiometric surface of the deep aquifer in the South Track, South Base, and Branch Park well-field area. A 15-foot decline in the potentiometric surface from April to September 1992 and 20- to 30-foot drawdowns in the three production wells in the South Track well field caused locally unconfined conditions in the deep aquifer.

\section{INTRODUCTION}

Land subsidence, resulting from aquifer-system compaction caused by declining ground-water levels, and the associated playa-surface deformation of Rogers Lake affect the strategic and economic operations at Edwards Air Force Base (EAFB), Antelope Valley, California (fig. 1). Deformation of the playa surface by land subsidence at Rogers Lake has caused sinklike depressions, fissures, and desiccation cracks that adversely affect the use of the playa as a runway (Blodgett and Williams, 1992; Londquist and others, 1993). The playa is used by the U.S. Department of the Air Force and the National Aeronautics and Space Administration for test aircraft and space shuttle landings.

A ground-water-level monitoring program was developed and maintained by the U.S. Geological Survey (USGS) in cooperation with the U.S. Department of the Air Force, Edwards Air Force Base, Air Force Flight Test Center during 1992 as part of a comprehensive investigation of land subsidence and aquifer-system compaction at EAFB. The objective of the comprehensive investigation is to determine the hydrologic factors related to land subsidence at EAFB and playa-surface deformation (Blodgett and Williams, 1992; Londquist and others, 1993). The data collected during this study and interpretations of these data will be needed in future work to determine 


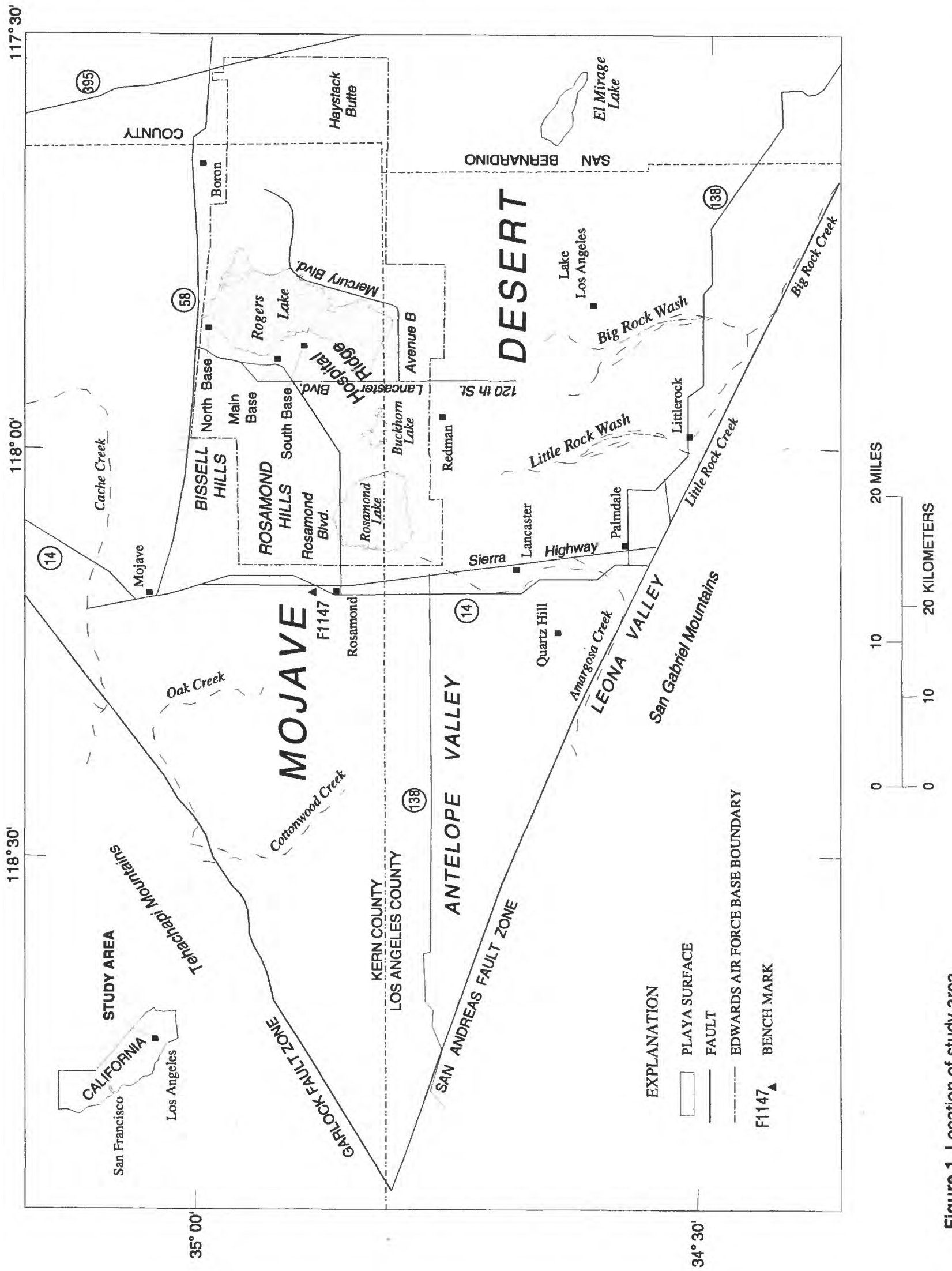

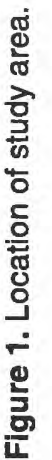


relations between declining ground-water levels and the distribution of land subsidence.

The ground-water-level monitoring program was implemented to monitor spatial and temporal changes in the potentiometric surfaces of the aquifer system. These changes are caused primarily by ground-water pumping. The program focused on areas of the base where ground-water pumping occurs, especially near Rogers Lake and areas that might be developed for future ground-water supply. This program establishes a baseline for future ground-water-level monitoring and aquifer restoration programs.

\section{Description of Study Area}

Edwards Air Force Base is about $60 \mathrm{mi}$ northeast of Los Angeles, in Antelope Valley, California. Antelope Valley is bounded by the Garlock Fault Zone and the Tehachapi Mountains to the west and northwest, the San Andreas Fault Zone and the San Gabriel Mountains to the south and southwest, and low bedrock hills to the east and north (fig. 2). Antelope Valley is in the rain shadow of the San Gabriel and Tehachapi Mountains. The climate at EAFB is arid with an average annual precipitation of $4.96 \mathrm{in}$. (period of record, 1942-92) (Donald Cameron, Range Staff Meteorologist, Air Force Flight Test Center, Edwards Air Force Base, written commun., 1993). Total precipitation in 1992 at the base was 12.07 in., 7.11 in. above average. The boundary of EAFB encompasses about $470 \mathrm{mi}^{2}$ of arkosic alluvium, low sand dunes, and playa surfaces surrounded by exposed bedrock hills (fig. 2).

\section{Purpose and Scope}

This report describes the ground-water-level monitoring program and presents interpretations of the ground-water-basin boundaries and seasonal potentiometric surfaces derived from the data collected during this monitoring program. Well-construction and historical water-level data were compiled for 118 wells and piezometers on and near the base. Monthly water-level measurements were made in 82 wells and piezometers on the base from January through December 1992. Land-surface altitudes for most of the monitored wells and piezometers were surveyed using differential leveling and Global Positioning System (GPS) surveying; some land-surface altitudes were derived from topographic maps. Hydraulic heads were computed from land-surface altitudes and water-level measurements. Monthly pumpage data were computed and tabulated from daily pumpage logs. These data are presented in data tables, hydrographs, bar graphs, and potentiometric-surface maps in this report.

\section{Acknowledgments}

The author would like to thank the many individuals and offices at EAFB that supported and contributed to this work. The Office of Programs and Plans Management at the Air Force Flight Test Center authorized security clearance into sensitive areas on EAFB and coordinated our activities on the base. The Civil Engineering office provided construction and pumpage data for production wells and access to pumphouses. The Base Operations office authorized access to Rogers Lake and provided meteorological data for EAFB. The Phillips Laboratory Civil Engineering office provided construction and pumpage data for production wells and escort into that facility.

\section{HYDROGEOLOGIC SETTING}

Antelope Valley is a closed alluvial basin filled with 5,000 to $10,000 \mathrm{ft}$ of sediment (Durbin, 1978). Twelve ground-water subbasins have been identified in the Antelope Valley (fig. 2) (Bloyd, 1967). The aquifer system at EAFB is part of two ground-water subbasins, the Lancaster subbasin and the North Muroc subbasin (fig. 3) (Bloyd, 1967; Londquist and others, 1993). The aquifer system in the Lancaster subbasin is divided into two aquifers, the unconfined principal aquifer which overlies the partly confined, deep aquifer (fig. 4). These two aquifers are separated by a southwestward-dipping confining unit consisting of blue or greenish-gray, fine- to very fine-grained lacustrine deposits of locally variable thickness. The confining unit is shallow along the southern shore of Rogers Lake where it is overlain by thin playa deposits. The aquifer in the North Muroc subbasin is unconfined. For a more thorough description of the areal extent of the confining unit, the reader is referred to Durbin (1978). Lithologies of these aquifers are described by Londquist and others (1993) and Rewis (1993). 


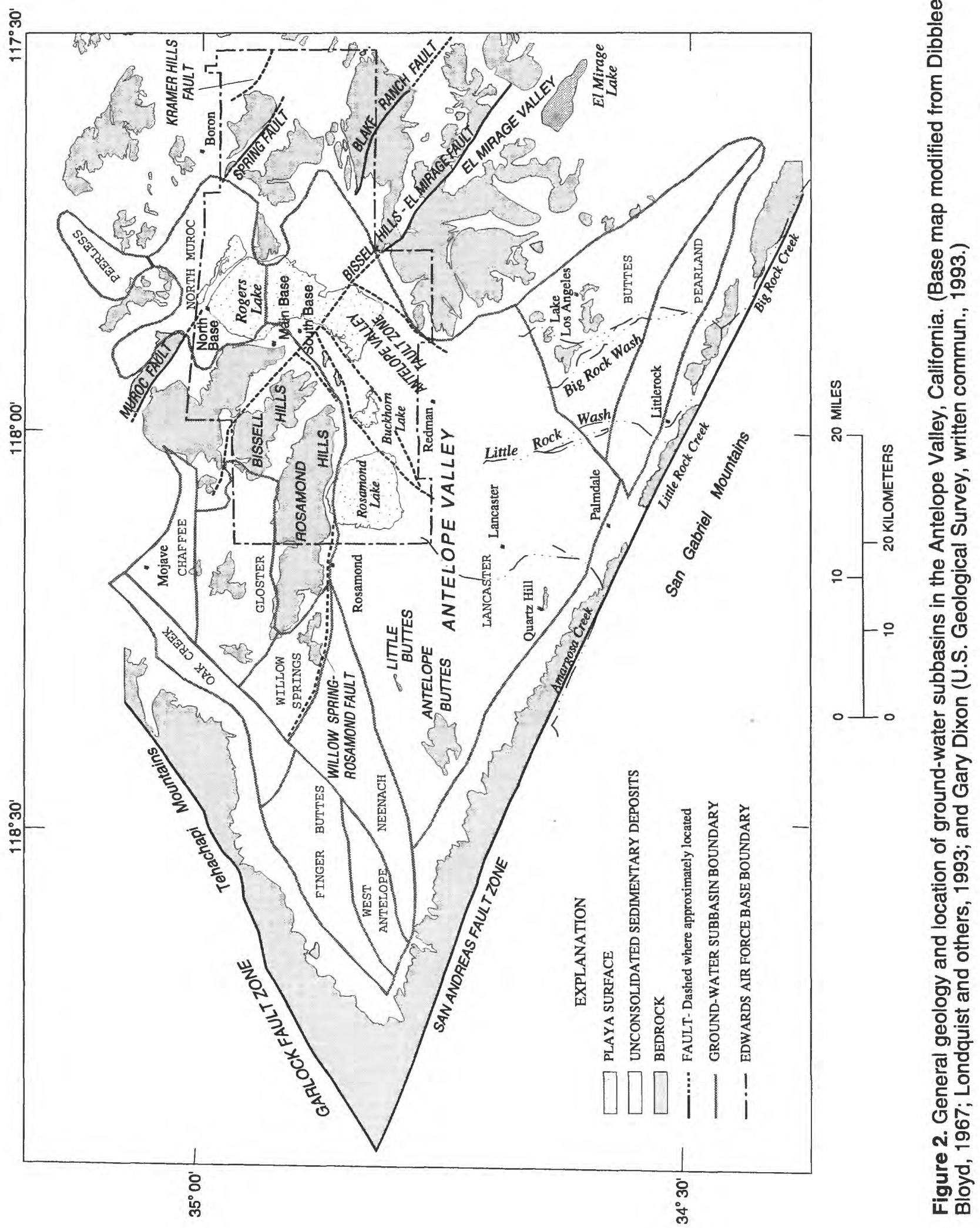



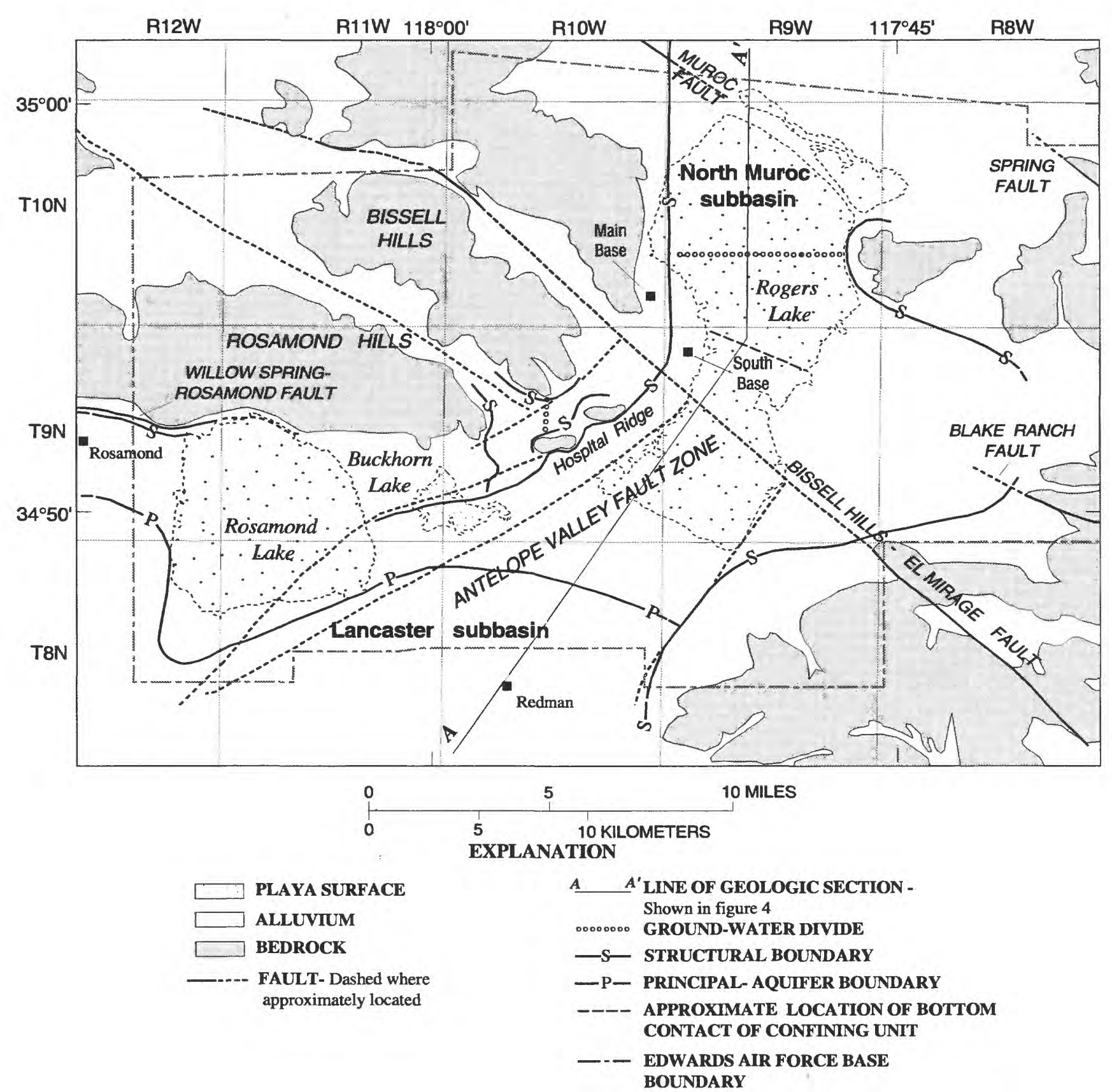

Figure 3. General geology and ground-water basin boundaries at Edwards Air Force Base, California, and geologic section $A-A^{\prime}$. (Base map modified from Dibblee, 1960; Bloyd, 1967; Londquist and others, 1993; and Gary Dixon (U.S. Geological Survey, written commun., 1993).

To map potentiometric surfaces and groundwater flow at EAFB, boundary conditions for the aquifer system had to be identified. Three types of no-flow boundaries were identified: (1) structural boundaries, (2) the principal-aquifer boundary, and (3) groundwater divides (fig. 3). These boundaries are discussed more fully in the "Basin Boundary" section of this report. Each of these no-flow boundaries represents a specified-flux boundary where the flux across the boundary is equal to zero ("flux" refers to the volume of fluid crossing a unit cross-sectional surface area per unit time) (Franke and others, 1987). Some of these boundaries may coincide with faults recently identified in this part of Antelope Valley (fig. 3).

For simplicity, structural boundaries and ground-water divides are assumed to be fixed boundaries for the period of this study. In reality, the groundwater divides and, to a lesser extent, the structural and 


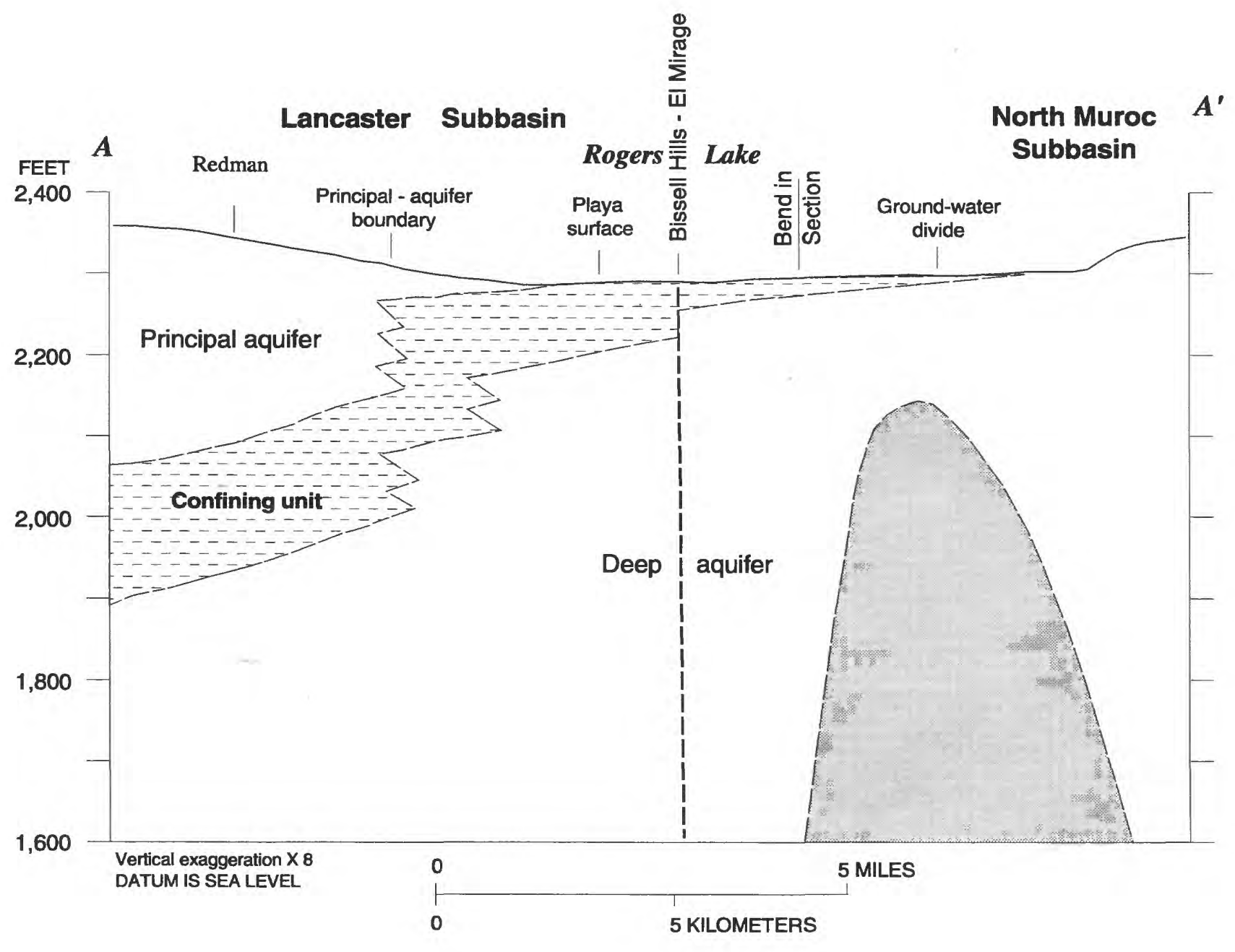

\section{EXPLANATION}

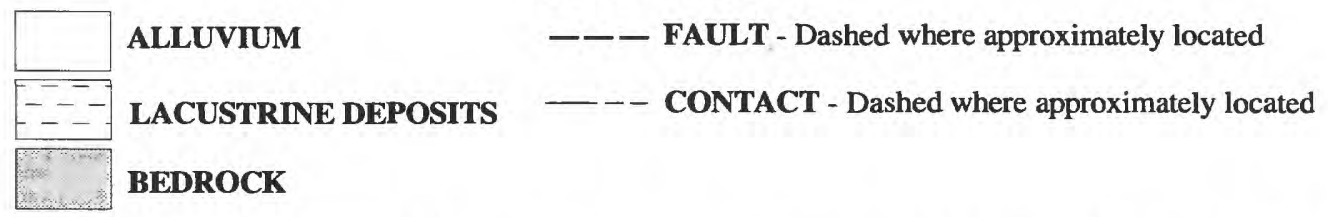

Figure 4. Geologic section $A-A^{\prime}$ showing the principal aquifer boundary and the ground-water divide between the Lancaster and North Muroc subbasins, Edwards Air Force Base, California.

principal-aquifer boundaries are time dependent and will migrate laterally with fluctuating ground-water levels.

Between Hospital Ridge and Rosamond and Bissell Hills lies a small, isolated, unnamed subbasin that previously has been included within the Lancaster subbasin boundary (Bloyd, 1967; Duell, 1987). The boundaries of the aquifer system in this area are not well defined. For purposes of this report, this small subbasin is considered separate from the Lancaster subbasin.

Average annual recharge to the aquifer system in Antelope Valley was estimated by Durbin (1978) to be 40,700 acre-ft, or 13,300 million gal. The principal source of recharge to the aquifer system in the Lancaster subbasin is infiltration of rainfall runoff through the alluvial fans of Big Rock, Little Rock, and Amargosa Creeks (fig. 1). Durbin (1978) reported that measured average annual runoff was 23,600 acre-ft, or 7.7 
billion gal, for the Big Rock Creek and Little Rock Creek drainage basins; he assumed that 100 percent of the runoff was recharge to the aquifer system. Snyder (1955) reported an annual runoff estimate of 3,584 acre- $\mathrm{ft}$, or 1.2 billion gal, for the Leona ValleyAmargosa Creek area.

Recharge to the North Muroc subbasin prior to development of the valley occurred as underflow from the Lancaster subbasin (Durbin, 1978). Because of pumping from the principal and deep aquifers, ground-water levels have declined in the Lancaster subbasin to the point where ground water no longer flows into the North Muroc subbasin (Durbin, 1978; Londquist and others, 1993). Ground-water-level data compiled for this study and presented in this report identify a ground-water divide hydraulically separating these two subbasins.

Recharge to the subbasins from infiltration in the bedrock hills on the eastern and northwestern parts of EAFB is minimal because average annual precipitation is less than $5 \mathrm{in} / \mathrm{yr}$, and average annual pan evaporation is high, about $114 \mathrm{in} / \mathrm{yr}$ (Bloyd, 1967). A small amount of runoff may infiltrate the alluvium along the base of the bedrock hills and the coarse-grained sediments along intermittent stream channels. Some direct recharge to the aquifer system within the valley from storm runoff was observed. This storm runoff inundated the playas and infiltrated the subsurface through giant desiccation cracks and fissures in the playa surface. The volume of this recharge is difficult to determine, but probably is small because the vertical pathways become plugged with low permeability sediments washed in from the surface. Most of the water that reaches the playa probably evaporates.

\section{GROUND-WATER-LEVEL MONITORING PROGRAM}

The ground-water-level monitoring program involved three phases of data collection: (1) well canvassing and selection, (2) geodetic surveys to determine vertical datum for each well, and (3) monthly water-level measurements. Ground-water levels in 82 wells and piezometers on EAFB (fig. 5) were measured monthly from January through December 1992. These included 48 piezometers that were installed by the USGS at 15 sites, 10 production wells in 7 well fields, 15 abandoned wells monitored by the USGS annually and semiannually as part of the Antelope Val-
ley-East Kern Water Agency ground-water-monitoring program, and 9 other abandoned homestead, irrigation, and production wells (table 1). Pumpage data were tabulated from daily records for the 10 production wells that were monitored at EAFB for this program and for 4 other production wells (9N/9W-14P2, $14 \mathrm{Q} 1,9 \mathrm{~N} / 10 \mathrm{~W}-34 \mathrm{P} 3$, and 10N/9W-5B1) that were not monitored for this program. These wells are included in table 1. Monthly pumpage totals were compared with water levels measured in the wells and piezometers in the base well fields.

Land-surface altitude, date of construction, and original ground-water-level data were compiled for 36 wells on and near the base that were not monitored for this program (table 1; fig. 5). Lithologic data for these wells were used to determine the position and extent of aquifer-system boundaries.

\section{Well Selection and Well Data}

Selection of wells used in the ground-waterlevel monitoring program was based on (1) measurable ground-water levels, (2) accessibility of the wells, (3) proximity to the base well fields and Rogers Lake, (4) proximity to other suitable wells to avoid redundancy, and (5) the position of the screened or perforated interval in the well. The wells were differentiated in table 1 as being completed within the deep aquifer, the principal aquifer, and the confining unit in the Lancaster subbasin; within the unconfined aquifer of the small, unnamed subbasin; near the groundwater divide on Rogers Lake; or within the unconfined aquifer in the North Muroc subbasin.

The USGS piezometers generally are single or nested, small, 2- to 3-inch diameter wells with 10- to 40-foot screens at isolated intervals in the borehole (table 1) (Londquist and others, 1993; Rewis, 1993). Piezometer 9N/10W-16F1 is 6.75 in. in diameter and is uncased and open to the bedrock formation in the interval from 275 to $458 \mathrm{ft}$ below land surface. The most shallow piezometers in the Lancaster subbasin are screened within the confining lacustrine unit and range from about 30 to $150 \mathrm{ft}$ below land surface. The deeper piezometers are screened in the deep or confined aquifer ranging from about 80 to $1,010 \mathrm{ft}$ below land surface. The tops of the screened or perforated intervals for most of the production and abandoned wells range from 96 to $300 \mathrm{ft}$ below land surface and 


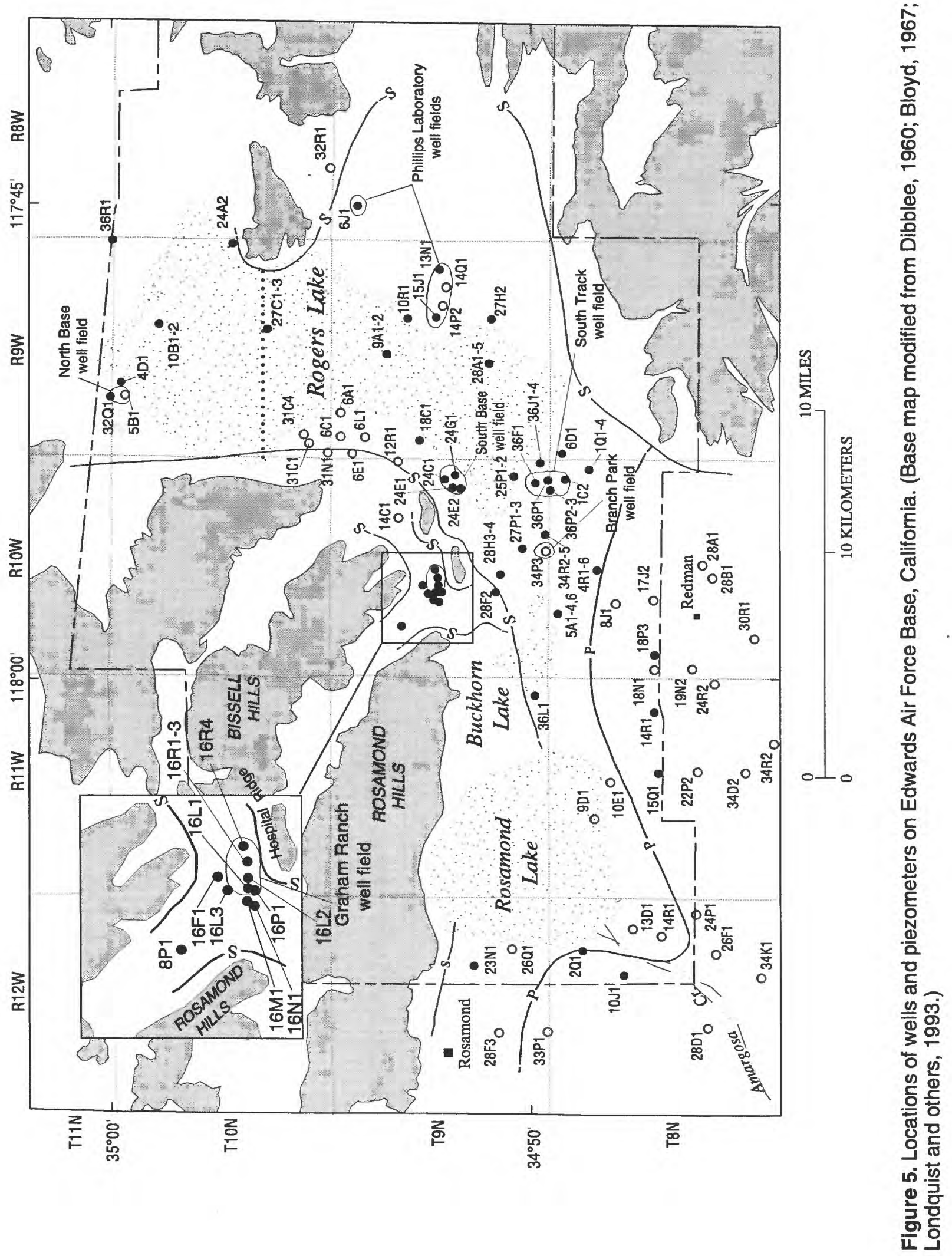




\section{EXPLANATION FOR FIGURE 5}

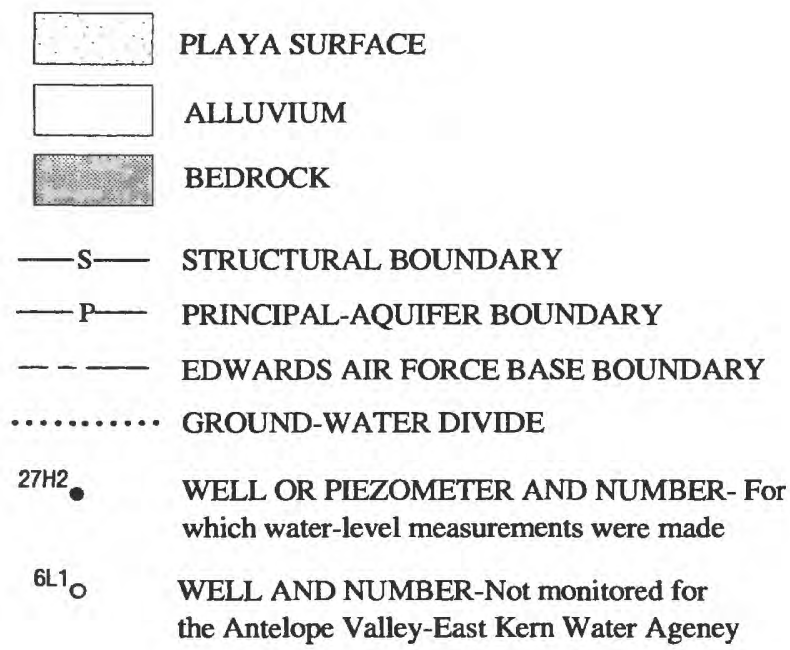

generally are screened to the bottom of the well (table 1).

\section{Land-Surface Altitudes}

Prior to this study, land-surface altitudes of wells on EAFB generally were not surveyed because of the remoteness of the well fields and homesteads. Land-surface altitudes were estimated from topographic maps with accuracies of about plus or minus one-half the contour interval of the map.

The accuracy of the land-surface altitude at a well is dependent on the method and precision standards used. Three methods were used to establish land-surface altitudes for the monitored wells and piezometers: third-order differential leveling; GPS surveying (J.C. Blodgett and M.E. Ikehara, U.S. Geological Survey, written commun., 1993); and estimates from USGS topographic quadrangle maps.

Spirit leveling surveys were made to 25 well and piezometer sites from bench marks along adjusted level lines that originated from bench mark F1147 (fig. 1). Bench mark F1147 was surveyed using first-order accuracy by the National Geodetic Survey in 1961 (U.S. Department of Commerce, 1966). Accuracy of the spirit leveling surveys at EAFB was plus or minus one-hundredth of a foot. Twenty-one stations, or sites, were measured using static and pseudo-kinematic GPS surveys. The average standard error for vertical components of the GPS surveys was about $0.1 \mathrm{ft}$. Because of adjustments along the level lines and the accuracy of the GPS surveys, land-surface altitudes derived from leveling and GPS surveys were rounded to the nearest tenth of a foot (table 1).

Land-surface altitudes for wells 8N/12W-2Q1, 9N/8W-6J1, and 9N/9W-13N1 (see fig. 5 for well locations) were estimated from 7.5-minute quadrangle maps of Rosamond Lake, Rogers Lake North, and Rogers Lake South. Accuracies of land-surface altitudes for these wells were assumed to be plus or minus one-half the contour interval, $2.5 \mathrm{ft}, 5 \mathrm{ft}$, and $10 \mathrm{ft}$, respectively.

\section{Ground-Water Levels and Hydraulic Heads}

Ground-water levels were measured to one-hundredth of a foot using a 300-foot calibrated steel measuring tape. Monthly ground-water levels in the wells and piezometers monitored for this study are listed in table 2 by well number and aquifer-system unit. Water levels generally ranged from about 95 to $130 \mathrm{ft}$ below land surface in the North Muroc subbasin, 70 to $200 \mathrm{ft}$ below land surface in the deep aquifer in the Lancaster subbasin, 35 to $95 \mathrm{ft}$ below land surface in the principal aquifer in the Lancaster subbasin, and 100 to $125 \mathrm{ft}$ below land surface in or near the Graham Ranch well field (table 2).

Hydraulic heads, or heads, were computed using ground-water levels and land-surface altitudes given in table 2. Head is the height of water in a well or piezometer referenced from an established datum, which for this report is sea level. Heads generally ranged from about 2,170 to $2,195 \mathrm{ft}$ above sea level in the North Muroc subbasin; 2,150 to $2,200 \mathrm{ft}$ above sea level in the deep aquifer in the Lancaster subbasin; 2,225 to $2,250 \mathrm{ft}$ above sea level in the principal aquifer in the Lancaster subbasin; and 2,200 to $2,215 \mathrm{ft}$ above sea level in the Graham Ranch well field. Heads in wells and piezometers completed in the confining unit ranged from about 2,210 to $2,275 \mathrm{ft}$ above sea level.

Seasonal fluctuations of hydraulic heads for USGS piezometers on or near the base well fields and on Rogers Lake are shown in figure 6. Seasonal fluctuations for heads in piezometers screened in the deep 
Table 1. Well-construction data and historic water-level data for wells and piezometers on and near Edwards Air Force Base, California

[State well No.: See well-numbering system on page V. See figure 5 for well locations. Altitude of land surface in feet above sea level rounded to the nearest tenth of a foot. Land-surface altitude, date of construction, and original ground-water-level data for wells not monitored for this study were compiled from Dutcher and others (1962). Type of well: AB, abandoned production or irrigation wells; AVEK, abandoned well monitored annually or semiannually by the U.S. Geological Survey for the Antelope Valley-East Kern Water Agency; D, destroyed; NPOT, production well for non-potable use; PIEZ, piezometer installed by the U.S. Geological Survey; POT, production well for potable use. Well depth, depth to water, and screened interval in feet below land surface. Casing in inches. --, data not available]

\begin{tabular}{|c|c|c|c|c|c|c|c|c|c|c|}
\hline \multirow{2}{*}{ State well No. } & \multirow{2}{*}{$\begin{array}{c}\text { Base } \\
\text { well } \\
\text { identifi- } \\
\text { cation } \\
\text { No. }\end{array}$} & \multirow{2}{*}{$\begin{array}{l}\text { Altitude } \\
\text { of land } \\
\text { surface }\end{array}$} & \multirow{2}{*}{$\begin{array}{l}\text { Type of } \\
\text { well }\end{array}$} & \multirow{2}{*}{$\begin{array}{l}\text { Date of } \\
\text { construc- } \\
\text { tion }\end{array}$} & \multicolumn{2}{|c|}{ Well depth } & \multirow{2}{*}{$\begin{array}{l}\text { Casing } \\
\text { diameter }\end{array}$} & \multirow{2}{*}{$\begin{array}{l}\text { Screened } \\
\text { interval }\end{array}$} & \multicolumn{2}{|c|}{$\begin{array}{l}\text { Earliest recorded } \\
\text { depth to water }\end{array}$} \\
\hline & & & & & Original & $\begin{array}{l}\text { Current } \\
\text { (1992) }\end{array}$ & & & Date & $\begin{array}{c}\text { Measure- } \\
\text { ment }\end{array}$ \\
\hline \multicolumn{11}{|c|}{ Completed in the deep aquifer in the Lancaster subbasin } \\
\hline $8 \mathrm{~N} / 10 \mathrm{~W}-1 \mathrm{C} 2$ & S-6 & $2,293.8$ & POT & 1984 & 700 & - & 16 & $300-690$ & $10 / 84$ & 146 \\
\hline$-1 Q 1$ & & $2,301.8$ & PIEZ & 1990 & 1,023 & 1,023 & 2 & $980-1,010$ & $5 / 90$ & 147.37 \\
\hline$-1 \mathrm{Q} 2$ & & $2,301.7$ & PIEZ & 1990 & 645 & 645 & 3 & $605-635$ & $5 / 90$ & 146.87 \\
\hline$-1 \mathrm{Q} 3$ & & $2,301.7$ & PIEZ & 1990 & 475 & 475 & 2 & $430-460$ & $5 / 90$ & 145.46 \\
\hline$-4 R 1$ & & $2,301.4$ & PIEZ & 1991 & 980 & 980 & 2 & $920-960$ & 7/91 & 146.40 \\
\hline$-4 R 2$ & & $2,301.4$ & PIEZ & 1991 & 750 & 750 & 2 & $700-740$ & $7 / 91$ & 145.17 \\
\hline$-4 R 3$ & & $2,301.4$ & PIEZ & 1991 & 546 & 546 & 2 & $496-536$ & $7 / 91$ & 143.36 \\
\hline$-4 R 4$ & & $2,301.4$ & PIEZ & 1991 & 250 & 250 & 2 & $220-240$ & $7 / 91$ & 135.11 \\
\hline$-5 \mathrm{Al}$ & & $2,287.3$ & PIEZ & 1989 & 947 & 947 & 2 & $897-927$ & $1 / 90$ & 129.65 \\
\hline$-5 \mathrm{~A} 2$ & & $2,287.3$ & PIEZ & 1991 & 560 & 560 & 2 & $530-550$ & $7 / 91$ & 127.02 \\
\hline$-5 \mathrm{~A} 3$ & & $2,287.3$ & PIEZ & 1991 & 390 & 390 & 2 & $360-380$ & $7 / 91$ & 127.11 \\
\hline$-5 \mathrm{~A} 4$ & & $2,287.3$ & PIEZ & 1991 & 274 & 274 & 2 & $246-266$ & $7 / 91$ & 121.99 \\
\hline$-30 \mathrm{R} 1^{1}$ & & 2,361 & AVEK & 1950 & 1,064 & 1,064 & 16 & $650-1,064$ & $2 / 73$ & 157.00 \\
\hline $8 \mathrm{~N} / 11 \mathrm{~W}-9 \mathrm{D} 1^{1}$ & & 2,276 & $\mathrm{D}$ & 1952 & 5,576 & -- & - & -- & -- & -- \\
\hline$-10 \mathrm{E} 1^{1}$ & & 2,289 & $\mathrm{AB}$ & -- & 612 & -- & 8 & $550-612$ & $5 / 51$ & 36.47 \\
\hline $8 \mathrm{~N} / 12 \mathrm{~W}-13 \mathrm{D} 1^{1}$ & & 2,283 & $\mathrm{AB}$ & 1949 & 451 & -- & 8 & $300-451$ & $11 / 52$ & 20.39 \\
\hline$-14 R 1^{1}$ & & 2,291 & $\mathrm{AB}$ & 1949 & 404 & - & 12 & $254-404$ & $11 / 51$ & 28.34 \\
\hline 9N/8W-6J1 & MW-3 & 2,394 & POT & 1961 & 363 & - & 14 & $147-363$ & $6 / 61$ & 145.7 \\
\hline $9 \mathrm{~N} / 9 \mathrm{~W}-6 \mathrm{~A} 1^{1}$ & & 2,275 & $\mathrm{AB}$ & 1943 & 199 & - & 14 & $76-184$ & -- & -- \\
\hline$-6 C 1^{1}$ & & 2,287 & $\mathrm{AB}$ & 1942 & 117 & -- & 14 & $38-101$ & $1 / 48$ & 39.8 \\
\hline$-6 \mathrm{E} 1^{1}$ & & 2,290 & $\mathrm{AB}$ & 1942 & 112 & - & 14 & $35-96$ & $1 / 48$ & 41.3 \\
\hline$-6 L 1^{1}$ & & 2,282 & $\mathrm{AB}$ & 1940 & 147 & - & 14 & $33-130$ & $1 / 48$ & 43.4 \\
\hline$-9 A 1$ & & $2,271.2$ & PIEZ & 1991 & 345 & 345 & 2 & $320-340$ & $7 / 91$ & 85.36 \\
\hline$-9 A 2$ & & $2,271.2$ & PIEZ & 1991 & 175 & 175 & 2 & $160-170$ & $7 / 91$ & 84.76 \\
\hline$-10 R 1$ & & $2,281.5$ & AVEK & 1937 & 106 & 97.9 & 9.5 & - & $10 / 51$ & 18.14 \\
\hline$-13 N 1$ & Well D & $2,350.2$ & POT & -- & 555 & - & 12 & $178-533$ & $8 / 62$ & 104.0 \\
\hline$-14 \mathrm{P} 2^{1}$ & Well B & 2,296 & POT & 1963 & 500 & - & 12 & - & $8 / 62$ & 53.0 \\
\hline$-14 Q 1^{1}$ & Well C & 2,320 & POT & -- & - & - & 12 & - & -- & -- \\
\hline$-15 \mathrm{~J} 1$ & Well A & $2,282.8$ & POT & -- & 534 & -- & 14 & $155-505$ & $8 / 62$ & 42 \\
\hline$-18 \mathrm{Cl}$ & S-1 & $2,280.1$ & $\mathrm{AB}$ & 1944 & 360 & 221 & 14 & $250-310$ & $1 / 48$ & 10.6 \\
\hline$-27 \mathrm{H} 2$ & & $2,279.8$ & AVEK & 1957 & 200 & 170.8 & 8 & $100-200$ & $7 / 57$ & 22.76 \\
\hline$-28 \mathrm{~A} 1$ & & $2,271.1$ & PIEZ & 1991 & 755 & 755 & 2 & $735-745$ & $6 / 91$ & 93.47 \\
\hline$-28 \mathrm{~A} 2$ & & $2,271.1$ & PIEZ & 1991 & 524 & 524 & 2 & $494-514$ & $6 / 91$ & 95.09 \\
\hline
\end{tabular}

Footnote at end of table. 
Table 1. Well-construction data and historic water-level data for wells and piezometers on and near Edwards Air Force Base, California--Continued

\begin{tabular}{|c|c|c|c|c|c|c|c|c|c|c|}
\hline \multirow[b]{2}{*}{ State well No. } & \multirow{2}{*}{$\begin{array}{c}\text { Base } \\
\text { well } \\
\text { identifi- } \\
\text { cation } \\
\text { No. }\end{array}$} & \multirow{2}{*}{$\begin{array}{c}\text { Altitude } \\
\text { of land } \\
\text { surface }\end{array}$} & \multirow{2}{*}{$\begin{array}{l}\text { Type of } \\
\text { well }\end{array}$} & \multirow{2}{*}{$\begin{array}{c}\text { Date of } \\
\text { construc- } \\
\text { tion }\end{array}$} & \multicolumn{2}{|c|}{ Well depth } & \multirow{2}{*}{$\begin{array}{l}\text { Casing } \\
\text { diameter }\end{array}$} & \multirow{2}{*}{$\begin{array}{l}\text { Screened } \\
\text { interval }\end{array}$} & \multicolumn{2}{|c|}{$\begin{array}{l}\text { Earliest recorded } \\
\text { depth to water }\end{array}$} \\
\hline & & & & & Original & $\begin{array}{c}\text { Current } \\
\text { (1992) }\end{array}$ & & & Date & $\begin{array}{c}\text { Measure- } \\
\text { ment }\end{array}$ \\
\hline \multicolumn{11}{|c|}{ Completed in the deep aquifer in the Lancaster subbasin--Continued } \\
\hline $9 \mathrm{~N} / 9 \mathrm{~W}-28 \mathrm{~A} 3$ & & $2,271.1$ & PIEZ & 1991 & 350 & 350 & 2 & $320-340$ & $6 / 91$ & 89.07 \\
\hline$-28 \mathrm{~A} 4$ & & $2,271.1$ & PIEZ & 1991 & 220 & 220 & 2 & $195-215$ & $6 / 91$ & 87.80 \\
\hline $9 \mathrm{~N} / 10 \mathrm{~W}-12 \mathrm{R} 1^{1}$ & & $2,280.7$ & $\mathrm{AB}$ & 1994 & 180 & 180 & 16 & -- & $1 / 48$ & 11.1 \\
\hline$-14 \mathrm{Cl}^{1}$ & & 2,288 & $\mathrm{AB}$ & 1942 & 113 & -- & 12 & $40-82$ & $1 / 42$ & 23.7 \\
\hline$-24 \mathrm{Cl}$ & S-9 & $2,283.0$ & AVEK & 1951 & 750 & 733 & 14 & $156-733$ & $7 / 52$ & 24.65 \\
\hline$-24 \mathrm{E} 1$ & S-11 & $2,271.9$ & $\mathrm{AB}$ & 1958 & 650 & -- & 16 & $280-650$ & $3 / 58$ & 29.38 \\
\hline$-24 \mathrm{E} 2$ & S-3 & $2,271.1$ & POT & 1974 & 590 & 579 & 14 & $220-590$ & $5 / 74$ & 116 \\
\hline$-24 \mathrm{Gl}$ & S-2 & $2,277.9$ & POT & 1951 & 738 & -- & 14 & $238-738$ & $10 / 51$ & 24.20 \\
\hline$-25 P 1$ & & $2,269.5$ & PIEZ & 1991 & 480 & 480 & 2 & $450-470$ & $11 / 91$ & 110.72 \\
\hline$-25 \mathrm{P} 2$ & & $2,269.5$ & PIEZ & 1991 & 130 & 130 & 2 & $100-120$ & $11 / 91$ & 71.60 \\
\hline$-27 P 1$ & & $2,278.6$ & PIEZ & 1992 & 560 & 560 & 3 & $530-550$ & $9 / 92$ & 127.65 \\
\hline$-27 \mathrm{P} 2$ & & $2,278.6$ & PIEZ & 1992 & 410 & 410 & 2 & $380-400$ & $9 / 92$ & 130.09 \\
\hline$-27 P 3$ & & $2,278.8$ & PIEZ & 1992 & 220 & 220 & 2 & $200-220$ & $9 / 92$ & 121.55 \\
\hline$-28 \mathrm{~F} 2$ & & $2,293.9$ & AVEK & 1953 & 140.8 & 140.8 & 10 & -- & $7 / 57$ & 44.55 \\
\hline$-28 \mathrm{H} 3$ & & $2,288.6$ & PIEZ & 1992 & 500 & 500 & 2 & $475-495$ & $9 / 92$ & 125.94 \\
\hline$-28 \mathrm{H} 4$ & & $2,288.6$ & PIEZ & 1992 & 305 & 305 & 2 & $275-295$ & $9 / 92$ & 131.92 \\
\hline$-34 \mathrm{P} 3^{1}$ & C-1 & 2,295 & NPOT & 1958 & 350 & -- & 8 & -- & -- & -- \\
\hline$-34 R 2$ & & $2,290.4$ & PIEZ & 1989 & 838 & 838 & 2 & $788-808$ & $1 / 91$ & 133.81 \\
\hline$-34 \mathrm{R} 3$ & & $2,290.0$ & PIEZ & 1989 & 520 & 520 & 2 & $480-510$ & $1 / 91$ & 132.80 \\
\hline$-34 R 4$ & & $2,290.0$ & PIEZ & 1989 & 250 & 250 & 2 & $210-240$ & $1 / 91$ & 132.08 \\
\hline$-36 \mathrm{~F} 1$ & S-4 & $2,285.6$ & POT & 1974 & 672 & -- & 14 & $216-662$ & -- & - \\
\hline$-36 \mathrm{~J} 1$ & & $2,283.0$ & PIEZ & 1991 & 900 & 900 & 2 & $870-890$ & $7 / 91$ & 127.70 \\
\hline$-36 \mathrm{~J} 2$ & & $2,283.0$ & PIEZ & 1991 & 529 & 529 & 2 & $503-523$ & $7 / 91$ & 131.92 \\
\hline$-36 \mathrm{~J} 3$ & & $2,283.0$ & PIEZ & 1991 & 237 & 237 & 2 & $212-232$ & $7 / 91$ & 125.31 \\
\hline$-36 \mathrm{P} 1$ & S-5 & $2,288.3$ & POT & 1974 & 667 & -- & 16 & $223-655$ & -- & -- \\
\hline$-36 \mathrm{P} 2$ & & $2,290.9$ & PIEZ & 1991 & 465 & 465 & 2 & $435-455$ & $11 / 91$ & 135.35 \\
\hline $9 \mathrm{~N} / 11 \mathrm{~W}-36 \mathrm{~L} 1$ & & $2,289.2$ & AVEK & -- & 127.1 & 127.1 & 12 & -- & $1 / 56$ & 30.86 \\
\hline $9 \mathrm{~N} / 12 \mathrm{~W}-23 \mathrm{~N} 1$ & & $2,292.4$ & AVEK & 1948 & 266.7 & 263.9 & 12 & -- & $3 / 51$ & 17.41 \\
\hline$-26 Q 1^{1}$ & & 2,286 & $\mathrm{AB}$ & 1945 & 300 & -- & 12 & $102-300$ & $3 / 47$ & flowing \\
\hline$-28 \mathrm{~F} 3^{1}$ & & 2,324 & $\mathrm{AB}$ & 1951 & 150 & 150 & 8 & -- & $8 / 51$ & 32.00 \\
\hline $10 \mathrm{~N} / 8 \mathrm{~W}-32 \mathrm{R} 1^{1}$ & & 2,450 & $\mathrm{AB}$ & 1948 & 148 & -- & -- & -- & -- & - \\
\hline $10 \mathrm{~N} / 9 \mathrm{~W}-31 \mathrm{C} 1^{1}$ & & 2,280 & $A B$ & -- & 177 & -- & 10 & -- & $1 / 51$ & 41.45 \\
\hline$-31 C 4^{1}$ & & 2,280 & $\mathrm{AB}$ & 1926 & 128 & -- & 16 & $48-114$ & $1 / 52$ & 44.72 \\
\hline$-31 N 1^{1}$ & & 2,294 & $\mathrm{AB}$ & 1948 & 83 & -- & 6 & $43-83$ & $11 / 51$ & 46.31 \\
\hline
\end{tabular}

Footnote at end of table. 
Table 1. Well-construction data and historic water-level data for wells and piezometers on and near Edwards Air Force Base, California--Continued

\begin{tabular}{|c|c|c|c|c|c|c|c|c|c|c|}
\hline \multirow{2}{*}{ State well No. } & \multirow{2}{*}{$\begin{array}{c}\text { Base } \\
\text { well } \\
\text { identifi- } \\
\text { cation } \\
\text { No. }\end{array}$} & \multirow{2}{*}{$\begin{array}{l}\text { Altitude } \\
\text { of land } \\
\text { şurface }\end{array}$} & \multirow{2}{*}{$\begin{array}{l}\text { Type of } \\
\text { well }\end{array}$} & \multirow{2}{*}{$\begin{array}{l}\text { Date of } \\
\text { construc- } \\
\text { tion }\end{array}$} & \multicolumn{2}{|c|}{ Well depth } & \multirow{2}{*}{$\begin{array}{l}\text { Casing } \\
\text { diameter }\end{array}$} & \multirow{2}{*}{$\begin{array}{l}\text { Screened } \\
\text { interval }\end{array}$} & \multicolumn{2}{|c|}{$\begin{array}{l}\text { Earliest recorded } \\
\text { depth to water }\end{array}$} \\
\hline & & & & & Original & $\begin{array}{l}\text { Current } \\
\text { (1992) }\end{array}$ & & & Date & $\begin{array}{l}\text { Measure- } \\
\text { ment }\end{array}$ \\
\hline \multicolumn{11}{|c|}{ Completed in the principal aquifer in the Lancaster subbasin } \\
\hline $8 \mathrm{~N} / 10 \mathrm{~W}-8 \mathrm{~J} 1^{1}$ & & 2,315 & $\overline{\mathrm{AB}}$ & 1951 & 648 & -- & 12 & - & $11 / 51$ & $\overline{63.83}$ \\
\hline$-17 \mathrm{~J}^{1}$ & & 2,327 & $\mathrm{AB}$ & -- & 206 & - & 12 & $110-206$ & $3 / 60$ & 61.40 \\
\hline$-18 N 1^{1}$ & & 2,324 & $\mathrm{AB}$ & 1919 & 275 & -- & 9 & $48-275$ & $3 / 49$ & 41.7 \\
\hline$-18 \mathrm{P} 3$ & & $2,322.5$ & $\mathrm{AB}$ & $\ldots$ & -- & 113.2 & 14 & - & - & - \\
\hline$-19 N 2^{1}$ & & 2,377 & $\mathrm{AB}$ & 1945 & 788 & - & 14 & $312-788$ & - & - \\
\hline$-28 \mathrm{~A} 1^{1}$ & & 2,359 & $\mathrm{AB}$ & 1945 & 288 & - & 12 & $102-186$ & -- & -- \\
\hline$-28 \mathrm{~B} 1^{1}$ & & 2,358 & AVEK & 1932 & 215 & - & 16 & - & $1 / 51$ & 66.75 \\
\hline $8 \mathrm{~N} / 11 \mathrm{~W}-14 \mathrm{R} 1$ & & $2,313.7$ & AVEK & 1949 & 186 & 164.9 & 12 & -- & $5 / 60$ & 93.11 \\
\hline$-15 Q 1$ & & $2,304.3$ & AVEK & 1952 & 179.2 & 177.8 & 12 & -- & $11 / 52$ & 77.98 \\
\hline$-22 \mathrm{P} 2^{1}$ & & 2,323 & AVEK & - & 202 & - & 12 & - & $12 / 78$ & 109.75 \\
\hline$-24 R 2^{1}$ & & 2,337 & AVEK & 1946 & 270 & 132.2 & 12 & $120-270$ & $5 / 51$ & 124.86 \\
\hline$-34 \mathrm{D} 2^{1}$ & & 2,340 & AVEK & $\ldots$ & 250 & 250 & 12 & - & $9 / 51$ & 145.8 \\
\hline$-34 R 2^{1}$ & & 2,358 & AVEK & - & 260 & - & 12 & - & $11 / 51$ & 147.71 \\
\hline $8 \mathrm{~N} / 12 \mathrm{~W}-2 \mathrm{Q} 1$ & & $2,283.8$ & AVEK & -- & 260 & 72.7 & 6 & - & $5 / 51$ & flowing \\
\hline$-10 \mathrm{~J} 1$ & & $2,288.8$ & AVEK & - & 91 & 85 & 6 & $30-91$ & $3 / 60$ & 13.67 \\
\hline$-24 P 1^{1}$ & & 2,307 & $\mathrm{AB}$ & 1923 & 723 & - & 18 & - & - & - \\
\hline$-26 \mathrm{~F} 1^{1}$ & & 2,303 & AVEK & - & 123 & 123 & 6 & - & $1 / 51$ & 14.27 \\
\hline$-28 \mathrm{D} 1^{1}$ & & 2,308 & AVEK & -- & 316 & 316 & 12 & $48-316$ & $4 / 51$ & 11.89 \\
\hline$-34 \mathrm{~K} 1^{1}$ & & 2,316 & AVEK & - & 144 & 144 & - & -- & - & - \\
\hline $9 \mathrm{~N} / 12 \mathrm{~W}-33 \mathrm{P} 1^{1}$ & & 2,310 & AVEK & -- & 146 & 146 & 12 & - & -- & -- \\
\hline \multicolumn{11}{|c|}{ Completed within the confining lacustrine unit in the Lancaster subbasin } \\
\hline $8 \mathrm{~N} / 9 \mathrm{~W}-6 \mathrm{D} 1$ & & $2,287.2$ & AVEK & 1950 & 200 & 135.6 & 8 & - & $3 / 59$ & 26.90 \\
\hline $8 \mathrm{~N} / 10 \mathrm{~W}-1 \mathrm{Q} 4$ & & $2,301.7$ & PIEZ & 1990 & 130 & 130 & 2 & $85-115$ & $5 / 90$ & 51.98 \\
\hline$-4 \mathrm{R} 5$ & & $2,301.4$ & PIEZ & 1991 & 150 & 150 & 2 & $135-150$ & $10 / 91$ & 89.54 \\
\hline$-4 R 6$ & & $2,301.4$ & PIEZ & 1991 & 100 & 100 & 2 & $80-100$ & $10 / 91$ & 59.56 \\
\hline$-5 A 6$ & & $2,287.3$ & PIEZ & 1991 & 55 & 55 & 2 & $30-50$ & $7 / 91$ & 28.02 \\
\hline 9N/9W-28A5 & & $2,271.1$ & PIEZ & 1991 & 65 & 65 & 3 & $40-60$ & 6/91 & 40.30 \\
\hline 9N/10W-34R5 & & $2,290.5$ & PIEZ & 1991 & 90 & 90 & 2 & $60-80$ & $10 / 91$ & 17.90 \\
\hline$-36 \mathrm{~J} 4$ & & $2,283.0$ & PIEZ & 1991 & 95 & 95 & 2 & $70-90$ & $7 / 91$ & 21.90 \\
\hline$-36 \mathrm{P} 3$ & & $2,291.2$ & PIEZ & 1991 & 120 & 120 & 2 & $90-110$ & $11 / 91$ & 27.88 \\
\hline \multicolumn{11}{|c|}{ Completed in the small, unconfined, unnamed subbasin } \\
\hline 9N/10W-8P1 & & $2,370.5$ & AVEK & -- & 137 & 132.6 & 6 & - & $10 / 51$ & 82.45 \\
\hline$-16 \mathrm{~F} 1$ & & $2,320.7$ & PIEZ & 1991 & 458 & 458 & 6.75 & $275-458$ & $1 / 92$ & 111.53 \\
\hline
\end{tabular}

Footnote at end of table. 
Table 1. Well-construction data and historic water-level data for wells and piezometers on and near Edwards Air Force Base, California--Continued

\begin{tabular}{|c|c|c|c|c|c|c|c|c|c|c|}
\hline \multirow{2}{*}{ State well No. } & \multirow{2}{*}{$\begin{array}{c}\text { Base } \\
\text { well } \\
\text { identifi- } \\
\text { cation } \\
\text { No. }\end{array}$} & \multirow{2}{*}{$\begin{array}{l}\text { Altitude } \\
\text { of land } \\
\text { surface }\end{array}$} & \multirow{2}{*}{$\begin{array}{l}\text { Type of } \\
\text { well }\end{array}$} & \multirow{2}{*}{$\begin{array}{l}\text { Date of } \\
\text { construc- } \\
\text { tion }\end{array}$} & \multicolumn{2}{|c|}{ Well depth } & \multirow{2}{*}{$\begin{array}{l}\text { Casing } \\
\text { diameter }\end{array}$} & \multirow{2}{*}{$\begin{array}{l}\text { Screened } \\
\text { interval }\end{array}$} & \multicolumn{2}{|c|}{$\begin{array}{l}\text { Earliest recorded } \\
\text { depth to water }\end{array}$} \\
\hline & & & & & Original & $\begin{array}{l}\text { Current } \\
\text { (1992) }\end{array}$ & & & Date & $\begin{array}{c}\text { Measure- } \\
\text { ment }\end{array}$ \\
\hline \multicolumn{11}{|c|}{ Completed in the small, unconfined, unnamed subbasin--Continued } \\
\hline $9 \mathrm{~N} / 10 \mathrm{~W}-16 \mathrm{~L} 1$ & & $2,319.5$ & $\mathrm{AB}$ & 1948 & 500 & -- & 14 & -- & $10 / 51$ & 125.20 \\
\hline$-16 L 2$ & & $2,319.0$ & $\mathrm{AB}$ & 1949 & 723 & -- & 14 & -- & $10 / 51$ & 96.82 \\
\hline$-16 \mathrm{~L} 3$ & & $2,318.7$ & $\mathrm{AB}$ & 1989 & 270 & 270 & 16 & $50-260$ & $1 / 90$ & 111.50 \\
\hline$-16 \mathrm{M} 1$ & & $2,324.0$ & $\mathrm{AB}$ & 1938 & 140.7 & 140.5 & 16 & -- & $2 / 51$ & 103.52 \\
\hline$-16 \mathrm{~N} 1$ & & $2,325.8$ & $\mathrm{AB}$ & 1946 & 396 & 376 & 14 & $96-396$ & $5 / 54$ & 99.39 \\
\hline$-16 \mathrm{P} 1$ & C-3 & $2,320.2$ & NPOT & 1949 & 532 & -- & 14 & $96-528$ & $4 / 52$ & 99.06 \\
\hline$-16 R 1$ & & $2,312.9$ & PIEZ & 1989 & 840 & 840 & 2 & $800-830$ & $3 / 90$ & 100.67 \\
\hline$-16 R 2$ & & $2,312.8$ & PIEZ & 1989 & 584 & 584 & 2 & $494-564$ & $3 / 90$ & 101.19 \\
\hline$-16 R 3$ & & $2,312.8$ & PIEZ & 1989 & 360 & 360 & 2 & $300-340$ & $3 / 90$ & 101.59 \\
\hline$-16 R 4$ & C-4 & $2,308.4$ & NPOT & 1990 & 700 & 700 & 16 & $290-690$ & $4 / 90$ & 110 \\
\hline \multicolumn{11}{|c|}{ Completed near the ground-water divide on Rogers Lake } \\
\hline $10 \mathrm{~N} / 9 \mathrm{~W}-27 \mathrm{Cl}$ & & $2,272.4$ & PIEZ & 1991 & 222 & 222 & 2 & $207-217$ & $7 / 91$ & $\overline{79.59}$ \\
\hline$-27 \mathrm{C} 2$ & & $2,272.4$ & PIEZ & 1991 & 160 & 160 & 2 & $130-150$ & $7 / 91$ & 78.39 \\
\hline$-27 \mathrm{C} 3$ & & $2,272.4$ & PIEZ & 1991 & 80 & 80 & 2 & $55-75$ & $7 / 91$ & 70.29 \\
\hline \multicolumn{11}{|c|}{ Completed in the unconfined aquifer in the North Muroc subbasin } \\
\hline $10 \mathrm{~N} / 9 \mathrm{~W}-4 \mathrm{D} 1$ & & $2,304.2$ & AVEK & 1957 & 502 & 456.2 & 12 & $144-433$ & $3 / 57$ & $\overline{95.02}$ \\
\hline$-5 B 1^{1}$ & $\mathrm{~N}-2$ & $2,278.0$ & POT & 1964 & 500 & -- & 16 & $100-500$ & $6 / 64$ & 75.99 \\
\hline$-10 \mathrm{~B} 1$ & & $2,278.6$ & PIEZ & 1991 & 312 & 312 & 2 & $285-302$ & $10 / 91$ & 95.42 \\
\hline$-10 \mathrm{~B} 2$ & & $2,278.6$ & PIEZ & 1991 & 150 & 150 & 2 & $117-137$ & $10 / 91$ & 95.10 \\
\hline$-24 \mathrm{~A} 2$ & & $2,290.6$ & AVEK & 1953 & -- & 278.7 & 14 & -- & $5 / 54$ & 72.56 \\
\hline $11 \mathrm{~N} / 9 \mathrm{~W}-32 \mathrm{Q} 1$ & $\mathrm{~N}-1$ & $2,302.9$ & $A B$ & 1957 & 450 & -- & 16 & $234-450$ & $10 / 57$ & 93.61 \\
\hline$-36 R 1$ & & $2,311.9$ & AVEK & 1953 & 298 & 254.1 & 10 & $100-132$ & $5 / 54$ & 98.25 \\
\hline
\end{tabular}

${ }^{1}$ Wells not monitored for this study. 
Table 2. Ground-water levels for wells and piezometers on Edwards Air Force Base, California, 1992

[Water level, in feet below land surface. Altitude: altitude of land surface in feet above sea level. Depth: depth to water in feet below land surface]

\begin{tabular}{|c|c|c|c|c|c|c|c|}
\hline Date & Water Level & Date & Water level & Date & Water level & Date & Water level \\
\hline \multicolumn{8}{|c|}{ Completed in deep aquifer in the Lancaster subbasin } \\
\hline \multicolumn{8}{|c|}{ 8N/10W-1C2. Altitude, 2,293.8; depth, 700} \\
\hline $1-09-92$ & 131.30 & $4-07-92$ & 130.81 & $8-06-92$ & 144.35 & $11-07-92$ & 135.62 \\
\hline $2-03-92$ & 130.47 & $6-16-92$ & 143.73 & $9-09-92$ & 144.73 & $12-17-92$ & 132.23 \\
\hline $3-10-92$ & 134.90 & $7-08-92$ & 140.40 & $10-07-92$ & 144.92 & & \\
\hline \multicolumn{8}{|c|}{ 8N/10W-1Q1. Altitude, 2,301.8; depth, 1,023 } \\
\hline $1-08-92$ & 144.42 & 4-04-92 & 142.46 & $7-13-92$ & 145.71 & $10-29-92$ & 147.86 \\
\hline $1-13-92$ & 144.36 & $5-05-92$ & 142.63 & $8-05-92$ & 146.62 & $12-14-92$ & 145.31 \\
\hline $2-03-92$ & 143.56 & $6-09-92$ & 144.89 & $9-09-92$ & 147.79 & & \\
\hline 2-24-92 & 142.85 & $6-10-92$ & 144.93 & $9-14-92$ & 147.79 & & \\
\hline $3-10-92$ & 142.60 & $7-07-92$ & 145.59 & $10-05-92$ & 147.82 & & \\
\hline \multicolumn{8}{|c|}{ 8N/10W-1Q2. Altitude, 2,301.7; depth, 645} \\
\hline $1-08-92$ & 137.55 & $4-04-92$ & 137.12 & $7-13-92$ & 145.43 & $10-05-92$ & 146.47 \\
\hline $1-13-92$ & 137.61 & $5-05-92$ & 142.42 & $7-14-92$ & 145.56 & $10-29-92$ & 143.66 \\
\hline $2-03-92$ & 136.95 & $6-09-92$ & 145.87 & $8-05-92$ & 146.18 & $12-14-92$ & 138.26 \\
\hline $2-24-92$ & 137.08 & $6-11-92$ & 144.48 & $9-09-92$ & 146.63 & & \\
\hline $3-10-92$ & 137.13 & $7-07-92$ & 145.29 & $9-14-92$ & 146.34 & & \\
\hline \multicolumn{8}{|c|}{ 8N/10W-1Q3. Altitude, 2,301.7; depth, 475} \\
\hline $1-07-92$ & 136.92 & 4-04-92 & 136.52 & $8-05-92$ & 145.28 & $11-07-92$ & 141.87 \\
\hline $1-13-92$ & 136.99 & $5-05-92$ & 141.21 & $9-09-92$ & 145.71 & $12-14-92$ & 137.66 \\
\hline $2-03-92$ & 136.39 & $6-09-92$ & 144.40 & 9-14-92 & 145.42 & & \\
\hline $2-24-92$ & 136.23 & $7-07-92$ & 144.35 & $10-05-92$ & 145.65 & & \\
\hline $3-10-92$ & 136.20 & $7-13-92$ & 144.56 & $10-29-92$ & 143.11 & & \\
\hline \multicolumn{8}{|c|}{ 8N/10W-4R1. Altitude, 2,301.4; depth, 980} \\
\hline $1-09-92$ & 146.71 & 4-05-92 & 143.66 & $7-08-92$ & 144.39 & $10-05-92$ & 146.93 \\
\hline $2-03-92$ & 145.83 & $5-07-92$ & 143.44 & $8-11-92$ & 145.36 & $11-08-92$ & 147.26 \\
\hline $3-09-92$ & 144.43 & $6-16-92$ & 144.31 & $9-09-92$ & 146.28 & $12-14-92$ & 146.56 \\
\hline \multicolumn{8}{|c|}{ 8N/10W-4R2. Altitude, 2,301.4; depth, 750} \\
\hline $1-09-92$ & 143.00 & 4-05-92 & 140.27 & $7-08-92$ & 143.36 & $10-05-92$ & 146.06 \\
\hline $2-03-92$ & 142.00 & $5-07-92$ & 140.89 & $8-11-92$ & 144.60 & $11-08-92$ & 145.64 \\
\hline $3-10-92$ & 140.59 & $6-16-92$ & 143.14 & $9-09-92$ & 145.70 & $12-14-92$ & 143.41 \\
\hline \multicolumn{8}{|c|}{ 8N/10W-4R3. Altitude, 2,301.4; depth, 546} \\
\hline $1-09-92$ & 140.59 & $4-05-92$ & 138.74 & $7-08-92$ & 141.78 & $10-05-92$ & 143.89 \\
\hline $2-03-92$ & 139.74 & $5-07-92$ & 139.70 & $8-11-92$ & 142.88 & $11-08-92$ & 142.93 \\
\hline $3-09-92$ & 138.53 & $6-16-92$ & 141.43 & $9-09-92$ & 143.67 & $12-14-92$ & 140.56 \\
\hline \multicolumn{8}{|c|}{ 8N/10W-4R4. Altitude, 2,301.4; depth, 250} \\
\hline $1-09-92$ & 133.66 & 4-05-92 & 132.04 & $7-08-92$ & 133.81 & $10-05-92$ & 135.51 \\
\hline $2-03-92$ & 132.96 & $5-07-92$ & 132.64 & $8-11-92$ & 134.68 & $11-08-92$ & 135.13 \\
\hline $3-09-92$ & 132.00 & $6-16-92$ & 133.58 & $9-09-92$ & 135.28 & $12-14-92$ & 133.65 \\
\hline
\end{tabular}


Table 2. Ground-water levels for wells and piezometers on Edwards Air Force Base, California, 1992--Continued

\begin{tabular}{|c|c|c|c|c|c|c|c|}
\hline Date & Water level & Date & Water level & Date & Water level & Date & Water level \\
\hline \multicolumn{8}{|c|}{ Completed in the deep aquifer in the Lancaster subbasin--Continued } \\
\hline \multicolumn{8}{|c|}{ 8N/10W-5A1. Altitude, 2,287.3; depth 947} \\
\hline $1-09-92$ & 127.23 & $3-31-92$ & 125.72 & $7-08-92$ & 125.21 & $10-05-92$ & 126.38 \\
\hline 2-03-92 & 126.79 & $5-08-92$ & 125.48 & $8-11-92$ & 125.73 & $11-08-92$ & 126.61 \\
\hline 3-09-92 & 126.18 & $6-16-92$ & 125.72 & $9-09-92$ & 126.16 & $12-14-92$ & 126.37 \\
\hline \multicolumn{8}{|c|}{ 8N/10W-5A2. Altitude, 2,287.3; depth, 560} \\
\hline $1-09-92$ & 126.44 & $3-31-92$ & 124.60 & $7-08-92$ & 125.56 & $10-05-92$ & 126.95 \\
\hline 2-03-92 & 125.80 & $5-08-92$ & 124.75 & $8-11-92$ & 126.18 & $11-08-92$ & 126.94 \\
\hline 3-09-92 & 124.93 & $6-16-92$ & 125.52 & $9-09-92$ & 126.74 & $12-14-92$ & 126.06 \\
\hline \multicolumn{8}{|c|}{ 8N/10W-5A3. Altitude, 2,287.3; depth, 390} \\
\hline $1-09-92$ & 126.39 & $3-31-92$ & 124.50 & $7-08-92$ & 125.68 & $10-05-92$ & 127.19 \\
\hline $2-03-92$ & 125.70 & $5-08-92$ & 124.75 & $8-11-92$ & 126.37 & $11-08-92$ & 127.10 \\
\hline $3-09-92$ & 124.80 & $6-16-92$ & 125.62 & $9-09-92$ & 126.96 & $12-14-92$ & 126.06 \\
\hline \multicolumn{8}{|c|}{ 8N/10W-5A4. Altitude, 2,287.3; depth 274} \\
\hline $1-09-92$ & 126.41 & $3-31-92$ & 124.48 & $7-08-92$ & 125.82 & $10-05-92$ & 127.37 \\
\hline 2-03-92 & 125.70 & $5-08-92$ & 124.80 & $8-11-92$ & 126.53 & $11-08-92$ & 127.22 \\
\hline $3-09-92$ & 124.77 & $6-16-92$ & 125.70 & $9-09-92$ & 127.14 & $12-14-92$ & 126.10 \\
\hline \multicolumn{8}{|c|}{ 9N/8W-6J1. Altitude, 2,394; depth, 363} \\
\hline $2-06-92$ & 202.76 & $6-17-92$ & 204.37 & $9-09-92$ & 204.39 & $12-17-92$ & 204.09 \\
\hline $3-13-92$ & 204.06 & $7-09-92$ & 204.31 & $10-08-92$ & 204.86 & & \\
\hline $5-09-92$ & 203.30 & 8-08-92 & 203.91 & $11-07-92$ & $227.54^{1}$ & & \\
\hline
\end{tabular}

\begin{tabular}{|c|c|c|c|c|c|c|c|}
\hline \multicolumn{8}{|c|}{ 9N/9W-9A1. Altitude, 2,271.2; depth, 345} \\
\hline $2-05-92$ & 88.40 & $5-11-92$ & 86.36 & $8-06-92$ & 86.30 & $11-07-92$ & 86.41 \\
\hline $3-11-92$ & 86.38 & $6-17-92$ & 86.50 & 9-09-92 & 86.46 & $12-16-92$ & 86.22 \\
\hline 4-05-92 & 86.52 & $7-07-92$ & 86.30 & $10-06-92$ & 86.56 & & \\
\hline \multicolumn{8}{|c|}{ 9N/9W-9A2. Altitude, 2,271.2; depth, 175} \\
\hline $2-05-92$ & 81.99 & $5-11-92$ & 82.19 & $8-06-92$ & 83.89 & $11-07-92$ & 84.22 \\
\hline $3-11-92$ & 82.41 & $6-17-92$ & 82.74 & 9-09-92 & 83.98 & $12-16-92$ & 84.06 \\
\hline 4-05-92 & 81.88 & $7-07-92$ & 83.63 & $10-06-92$ & 84.21 & & \\
\hline \multicolumn{8}{|c|}{ 9N/9W-10R1. Altitude, 2,281.5; depth 97.9} \\
\hline $2-05-92$ & 94.58 & $5-11-92$ & 94.98 & $8-06-92$ & 95.28 & $11-07-92$ & 95.49 \\
\hline $3-11-92$ & 94.75 & $6-17-92$ & 95.04 & 9-09-92 & 95.52 & $12-16-92$ & 95.72 \\
\hline 4-05-92 & 94.76 & $7-07-92$ & 95.15 & $10-06-92$ & 95.50 & & \\
\hline \multicolumn{8}{|c|}{ 9N/9W-13N1. Altitude, 2,350.2; depth, 555} \\
\hline $2-05-92$ & 161.39 & $6-17-92$ & 161.77 & $9-10-92$ & 162.35 & $12-17-92$ & 162.64 \\
\hline 3-13-92 & 161.63 & $7-09-92$ & 162.10 & $10-08-92$ & 162.43 & & \\
\hline $5-09-92$ & 161.54 & $8-08-92$ & 162.06 & $11-07-92$ & 162.33 & & \\
\hline \multicolumn{8}{|c|}{ 9N/9W-15J1. Altitude, 2,282.8; depth, 534} \\
\hline $2-06-92$ & 97.03 & $5-09-92$ & 97.11 & 8-08-92 & 97.82 & $11-07-92$ & 98.20 \\
\hline $3-11-92$ & 96.07 & $6-17-92$ & 96.80 & $9-10-92$ & 98.10 & $12-17-92$ & 97.94 \\
\hline 4-07-92 & 97.36 & 7-09-92 & 97.84 & $10-08-92$ & 98.09 & & \\
\hline
\end{tabular}

Footnote at end of table. 
Table 2. Ground-water levels for wells and piezometers on Edwards Air Force Base, California, 1992--Continued

\begin{tabular}{cccccccc}
\hline Date & Water level & Date & Water level & Date & Water level & Date & Water level \\
\hline \multicolumn{7}{c}{ Completed in the deep aquifer in the Lancaster subbasin--Continued } \\
\hline \multicolumn{7}{c}{ 9N/9W-18C1. Altitude, 2,280.1; depth, 221 } \\
\hline $2-04-92$ & 101.42 & $5-07-92$ & 101.29 & $8-10-92$ & 102.05 & $11-08-92$ & 102.08 \\
$3-10-92$ & 101.28 & $6-16-92$ & 101.66 & $9-09-92$ & 102.22 & $12-15-92$ & 101.81 \\
$4-05-02$ & 101.11 & $7-08-12$ & 101.75 & $10-07-92$ & 101.78 & &
\end{tabular}

\begin{tabular}{llllllll}
\hline \multicolumn{7}{c}{ 9N/9W-27H2. Altitude, 2,279.8; depth, 170.8 } \\
\hline $3-11-92$ & 93.50 & $6-17-92$ & 93.87 & $9-09-92$ & 94.21 & $12-16-92$ & 94.51 \\
$4-05-92$ & 93.52 & $7-09-92$ & 94.06 & $10-06-92$ & 94.28 & & \\
$5-09-92$ & 93.55 & $8-08-92$ & 94.17 & $11-07-92$ & 94.39 &
\end{tabular}

\begin{tabular}{llllllll}
\hline \multicolumn{8}{c}{ 9N/9W-28A1. Altitude, 2,271.1; depth 755 } \\
\hline $1-10-92$ & 92.49 & $4-05-92$ & 92.18 & $7-29-92$ & 94.94 & $10-28-92$ & 94.81 \\
$1-17-92$ & 92.39 & $5-05-92$ & 93.36 & $8-06-92$ & 94.98 & $12-17-92$ & 93.02 \\
$2-05-92$ & 92.24 & $6-09-92$ & 94.44 & $9-09-92$ & 95.23 & & \\
$3-09-92$ & 92.01 & $7-07-92$ & 94.39 & $9-14-92$ & 95.03 & & \\
$3-11-92$ & 92.11 & $7-13-92$ & 94.57 & $10-06-92$ & 95.34 &
\end{tabular}

\begin{tabular}{llllllll}
\hline \multicolumn{7}{c}{ 9N/9W-28A2. Altitude, 2,271.1; depth, 524 } \\
\hline $1-10-92$ & 91.32 & $4-05-92$ & 91.07 & $7-30-92$ & 93.49 & $10-06-92$ & 93.89 \\
$1-17-92$ & 91.22 & $5-05-92$ & 92.14 & $8-06-92$ & 93.54 & $10-28-92$ & 93.39 \\
$2-05-92$ & 91.08 & $6-09-92$ & 93.10 & $8-11-92$ & 93.54 & $11-07-92$ & 93.02 \\
$3-09-92$ & 90.93 & $7-07-92$ & 93.02 & $9-09-92$ & 93.81 & $12-16-92$ & 92.00 \\
$3-11-92$ & 91.01 & $7-13-92$ & 93.20 & $9-14-92$ & 93.65 & &
\end{tabular}

\begin{tabular}{rlllllrr}
\hline \multicolumn{7}{c}{ 9N/9W-28A3. Altitude, 2,271.1; depth, 350 } \\
\hline $1-17-92$ & 88.40 & $4-05-92$ & 88.34 & $7-13-92$ & 89.59 & $9-14-92$ & 89.97 \\
$2-05-92$ & 88.35 & $5-05-92$ & 88.86 & $7-30-92$ & 89.84 & $10-06-92$ & 90.16 \\
$3-09-92$ & 88.28 & $6-09-92$ & 89.46 & $8-06-92$ & 89.84 & $10-28-92$ & 89.95 \\
$3-11-92$ & 88.32 & $7-07-92$ & 89.50 & $9-09-92$ & 90.07 & $12-17-92$ & 89.14 \\
\hline \multicolumn{8}{c}{ 9N/9W-28A4. Altitude, 2,271.1; depth, 220 } \\
\hline $1-17-92$ & 87.26 & $5-05-92$ & 87.84 & $8-06-92$ & 88.76 & $11-07-92$ & 88.86 \\
$2-05-92$ & 87.50 & $6-09-92$ & 88.33 & $9-09-92$ & 88.96 & $12-16-92$ & 88.37 \\
$3-09-92$ & 87.34 & $7-07-92$ & 88.42 & $9-14-92$ & 88.87 & & \\
$3-11-92$ & 87.46 & $7-13-92$ & 88.52 & $10-06-92$ & 89.07 & & \\
$4-05-92$ & 87.44 & $7-30-92$ & 88.72 & $10-28-92$ & 88.92 &
\end{tabular}

\begin{tabular}{|c|c|c|c|c|c|c|c|}
\hline \multicolumn{8}{|c|}{ 9N/10W-24C1. Altitude, 2,283.0; depth, 733} \\
\hline $1-10-92$ & 116.99 & $4-05-92$ & 115.87 & $7-08-92$ & 117.80 & $10-07-92$ & 118.94 \\
\hline $2-04-92$ & 116.41 & $5-07-92$ & 115.80 & $8-10-92$ & 118.49 & $11-08-92$ & 116.35 \\
\hline $3-10-92$ & 116.21 & $6-16-92$ & 117.25 & 9-09-92 & 118.59 & $12-17-92$ & 116.62 \\
\hline \multicolumn{8}{|c|}{ 9N/10W-24E1. Altitude, 2,271.9; depth, 650} \\
\hline $2-04-92$ & 109.37 & $5-07-92$ & 110.10 & $8-10-92$ & 135.44 & $11-08-92$ & 133.52 \\
\hline $3-10-92$ & 108.93 & $6-16-92$ & 133.60 & 9-09-92 & 128.06 & $12-17-92$ & 110.32 \\
\hline 4-05-92 & 111.26 & $7-08-92$ & 130.14 & $10-07-92$ & 126.28 & & \\
\hline
\end{tabular}


Table 2. Ground-water levels for wells and piezometers on Edwards Air Force Base, California, 1992--Continued

\begin{tabular}{|c|c|c|c|c|c|c|c|}
\hline Date & Water level & Date & Water level & Date & Water level & Date & Water level \\
\hline \multicolumn{8}{|c|}{ Completed in the deep aquifer in the Lancaster subbasin--Continued } \\
\hline \multicolumn{8}{|c|}{ 9N/10W-24E2. Altitude, 2,271.1; depth, 579} \\
\hline $5-07-92$ & 112.30 & $10-07-92$ & $182.42^{1}$ & $11-08-92$ & $237.21^{1}$ & $12-17-92$ & 109.45 \\
\hline \multicolumn{8}{|c|}{ 9N/10W-24G1. Altitude, 2,277.9; depth, 738} \\
\hline $2-04-92$ & $162.12^{1}$ & $7-08-95$ & $161.83^{1}$ & $9-09-92$ & $161.91^{1}$ & $12-17-92$ & 117.36 \\
\hline $5-07-92$ & $172.18^{1}$ & $8-10-92$ & $161.85^{1}$ & $11-08-92$ & 112.05 & & \\
\hline \multicolumn{8}{|c|}{ 9N/10W-25P1. Altitude, 2,269.5; depth, 480} \\
\hline $1-09-92$ & 106.07 & $5-09-92$ & 109.07 & $8-\sqrt[6-92]{2}$ & 114.77 & $10-07-92$ & 114.91 \\
\hline $2-03-92$ & 105.47 & $6-16-92$ & 114.28 & $9-09-92$ & 115.11 & $10-30-92$ & 111.61 \\
\hline $4-07-92$ & 105.62 & $7-08-92$ & 114.45 & $9-18-92$ & 113.29 & $12-15-92$ & 106.67 \\
\hline \multicolumn{8}{|c|}{ 9N/10W-25P2. Altitude, 2,269.5; depth, 130} \\
\hline $1-09-92$ & 69.91 & $5-09-92$ & 70.79 & $8-06-92$ & 71.77 & $10-07-92$ & 72.26 \\
\hline 2-03-92 & 70.07 & $6-16-92$ & 71.97 & $9-09-92$ & 72.11 & $10-30-92$ & 72.02 \\
\hline 4-07-92 & 70.06 & 7-08-92 & 71.32 & 9-18-92 & 72.28 & $12-15-92$ & 71.57 \\
\hline \multicolumn{8}{|c|}{ 9N/10W-27P1. Altitude, 2,278.6; depth, 560} \\
\hline $9-07-92$ & 127.65 & $10-05-92$ & 127.99 & $11-09-92$ & 128.17 & $12-16-92$ & 128.23 \\
\hline \multicolumn{8}{|c|}{ 9N/10W-27P2. Altitude, 2,278.6; depth, 410} \\
\hline $9-07-92$ & 130.09 & $10-05-92$ & 130.12 & $11-09-92$ & 129.67 & $12-16-92$ & 128.10 \\
\hline \multicolumn{8}{|c|}{ 9N/10W-27P3. Altitude, 2,278.8; depth, 220} \\
\hline $9-07-92$ & 121.55 & $10-05-92$ & 121.83 & $11-09-92$ & 121.31 & $12-16-92$ & 120.22 \\
\hline \multicolumn{8}{|c|}{ 9N/10W-28F2. Altitude, 2,293.9; depth, 140.8} \\
\hline $1-09-92$ & 90.55 & 4-04-92 & 90.56 & $7-07-92$ & 90.52 & $10-05-92$ & 90.84 \\
\hline 2-04-92 & 90.49 & $5-11-92$ & 90.65 & $8-10-92$ & 90.71 & $11-08-92$ & 90.81 \\
\hline $3-10-92$ & 90.63 & $6-16-92$ & 90.73 & $9-07-92$ & 90.78 & $12-15-92$ & 90.89 \\
\hline \multicolumn{8}{|c|}{ 9N/10W-28H3. Altitude, 2,288.6; depth, 500} \\
\hline $9-07-92$ & 125.94 & $10-05-92$ & 128.15 & $11-08-92$ & 127.78 & $12-16-92$ & 127.32 \\
\hline \multicolumn{8}{|c|}{ 9N/10W-28H4. Altitude, 2,288.6; depth, 305} \\
\hline $9-07-92$ & 131.92 & $10-05-92$ & 133.10 & $11-09-92$ & 133.25 & & \\
\hline \multicolumn{8}{|c|}{ 9N/10W-34R2. Altitude, 2,290.4; depth, 838} \\
\hline $2-03-92$ & 132.00 & $6-16-92$ & 134.92 & $9-17-92$ & 136.93 & $12-14-92$ & 133.40 \\
\hline 3-09-92 & 130.90 & 7-08-92 & 134.98 & $10-05-92$ & 137.20 & & \\
\hline 4-05-92 & 130.86 & $8-11-92$ & 136.22 & $10-29-92$ & 136.84 & & \\
\hline $5-08-92$ & 132.36 & 9-07-92 & 137.02 & $11-08-92$ & 136.11 & & \\
\hline \multicolumn{8}{|c|}{ 9N/10W-34R3. Altitude, 2,290.0; depth, 520} \\
\hline $1-10-92$ & 132.14 & 5-08-92 & 134.10 & $9-07-92$ & 136.77 & $12-15-92$ & 132.05 \\
\hline $2-03-92$ & 131.03 & $6-16-92$ & 134.82 & $9-17-92$ & 136.42 & & \\
\hline $3-09-92$ & 130.36 & 7-08-92 & 135.25 & $10-05-92$ & 136.98 & & \\
\hline $4-05-92$ & 130.66 & $8-11-92$ & 136.31 & $10-29-92$ & 135.78 & & \\
\hline
\end{tabular}

Footnote at end of table. 
Table 2. Ground-water levels for wells and piezometers on Edwards Air Force Base, California, 1992--Continued

\begin{tabular}{cccccccc}
\hline Date & Water level & Date & Water level & Date & Water level & Date & Water level \\
\hline \multicolumn{7}{c}{ Completed in the deep aquifer in the Lancaster subbasin-Continued } \\
\hline \multicolumn{7}{c}{ 9N/10W-34R4. Altitude, 2,290.0; depth, 250 } \\
\hline $3-03-92$ & 130.43 & $6-16-92$ & 134.20 & $9-17-92$ & 136.21 & $12-14-92$ & 131.62 \\
$4-05-92$ & 129.73 & $7-08-92$ & 134.66 & $10-05-92$ & 136.69 & & \\
$5-08-92$ & 130.42 & $8-11-92$ & 135.99 & $10-29-92$ & 135.47 & \\
& 132.63 & $9-07-92$ & 136.46 & $11-08-92$ & 134.76 &
\end{tabular}

\begin{tabular}{cccccccc}
\hline \multicolumn{8}{c}{ 9N/10W-36F1. Altitude, 2,825.6; depth, 672 } \\
\hline $1-09-92$ & 122.36 & $4-07-92$ & 122.27 & $8-06-92$ & $151.68^{1}$ & $11-07-92$ & 126.74 \\
$2-03-92$ & 141.85 & $5-09-92$ & 126.15 & $9-09-92$ & 133.50 & $12-17-92$ & 125.44 \\
$3-10-92$ & 124.22 & $6-16-92$ & $152.50^{1}$ & $10-07-92$ & 133.91 & &
\end{tabular}

\begin{tabular}{llllllll}
\hline \multicolumn{8}{c}{ 9N/10W-36J1. Altitude, 2,283.0; depth, 900 } \\
\hline $1-09-92$ & 122.28 & $5-09-92$ & 122.72 & $7-31-92$ & 128.30 & $10-05-92$ & 128.74 \\
$2-04-92$ & 121.30 & $6-16-92$ & 126.48 & $8-06-92$ & 128.65 & $10-30-92$ & 128.40 \\
$3-10-92$ & 120.57 & $7-08-92$ & 127.17 & $9-09-92$ & 129.60 & $12-15-92$ & 123.52 \\
$4-07-92$ & 120.64 & $7-16-92$ & 127.32 & $9-16-92$ & 129.35 & &
\end{tabular}

\begin{tabular}{llllllll}
\hline \multicolumn{8}{c}{ 9N/10W-36J2. Altitude, 2,283.0, depth, 529 } \\
\hline $1-09-92$ & 119.43 & $5-09-92$ & 124.12 & $7-31-92$ & 132.88 & $10-05-92$ & 131.55 \\
$2-04-92$ & 119.10 & $6-16-92$ & 131.73 & $8-06-92$ & 131.81 & $10-30-92$ & 125.07 \\
$3-10-92$ & 121.96 & $7-08-92$ & 130.35 & $9-09-92$ & 131.79 & $12-15-92$ & 120.02 \\
$4-07-92$ & 119.35 & $7-16-92$ & 131.23 & $9-16-92$ & 129.82 & &
\end{tabular}

\begin{tabular}{|c|c|c|c|c|c|c|c|}
\hline \multicolumn{8}{|c|}{ 9N/10W-36J3. Altitude, 2,283.0; depth, 237} \\
\hline $1-09-92$ & 116.54 & $5-09-92$ & 118.91 & $8-06-92$ & 124.44 & $11-07-92$ & 120.33 \\
\hline $2-04-92$ & 116.49 & $6-16-92$ & 124.67 & $9-09-92$ & 124.60 & $12-16-92$ & 117.25 \\
\hline $3-10-92$ & 116.59 & $7-08-92$ & 125.11 & $9-16-92$ & 125.30 & & \\
\hline 4-07-92 & 116.82 & $7-31-92$ & 125.64 & $10-07-92$ & 124.68 & & \\
\hline \multicolumn{8}{|c|}{ 9N/10W-36P1. Altitude, 2,288.3; depth, 667} \\
\hline $1-09-92$ & 126.41 & $5-09-92$ & $134 . \overline{05}$ & $8-06-92$ & $167.73^{1}$ & $11-07-92$ & 130.16 \\
\hline $2-03-92$ & 125.32 & $6-16-92$ & $167.90^{1}$ & $9-09-92$ & $170.35^{1}$ & $12-17-92$ & 127.51 \\
\hline 4-07-92 & 125.47 & $7-08-92$ & 137.27 & $10-07-92$ & $169.82^{1}$ & & \\
\hline \multicolumn{8}{|c|}{ 9N/10W-36P2. Altitude, 2,290.9; depth, 465} \\
\hline $1-09-92$ & 128.34 & $5-09-92$ & 136.92 & $7-29-92$ & 143.50 & $10-07-92$ & 139.42 \\
\hline $2-03-92$ & 127.22 & $6-16-92$ & 142.81 & $8-06-92$ & 142.21 & $10-30-92$ & 133.37 \\
\hline $3-10-92$ & 132.42 & $7-08-92$ & 138.10 & $9-09-92$ & 141.80 & $12-15-92$ & 128.16 \\
\hline $4-07-92$ & 127.48 & $7-15-92$ & 138.63 & $9-15-92$ & 142.17 & & \\
\hline \multicolumn{8}{|c|}{ 9N/11W-36L1. Altitude, 2,289.2; depth, 127.1} \\
\hline 4-06-92 & 92.19 & $7-08-92$ & 100.23 & $10-05-92$ & 99.88 & & \\
\hline $5-11-92$ & 101.47 & $8-05-92$ & 94.97 & $11-08-92$ & 101.54 & & \\
\hline $6-17-92$ & 100.11 & $9-07-92$ & 99.99 & $12-15-92$ & 93.13 & & \\
\hline
\end{tabular}

Footnote at end of table. 
Table 2. Ground-water levels for wells and piezometers on Edwards Air Force Base, California, 1992--Continued

\begin{tabular}{cccccccc}
\hline Date & Water level & Date & Water level & Date & Water level & Date & Water level \\
\hline \multicolumn{7}{c}{ Completed in the deep aquifer in the Lancaster subbasin--Continued } \\
\hline \multicolumn{7}{c}{ 9N/12 W-23N1. Altitude, 2,292.4; depth 263.9 } \\
\hline $3-12-92$ & 72.69 & $5-06-92$ & 72.56 & $8-05-92$ & 72.77 & $11-08-92$ & 73.15 \\
$4-06-92$ & 72.64 & $6-17-92$ & 72.65 & $9-09-92$ & 72.94 & $12-14-92$ & 73.35 \\
$4-16-92$ & 72.61 & $7-08-92$ & 72.65 & $10-05-92$ & 73.08 & &
\end{tabular}

\begin{tabular}{llllllll}
\hline \multicolumn{7}{c}{ Completed in the principal aquifer in the Lancaster subbasin } \\
\hline \multicolumn{7}{c}{ 8N/10W-18P3. Altitude, 2,322.5; depth, 113.2 } \\
\hline $2-07-92$ & 93.28 & $5-06-92$ & 93.46 & $8-05-92$ & 93.57 & $11-08-92$ & 93.62 \\
$3-12-92$ & 93.39 & $6-17-92$ & 93.54 & $9-09-92$ & 93.62 & $12-14-92$ & 93.70 \\
$3-31-92$ & 93.42 & $7-08-92$ & 93.57 & $10-05-92$ & 93.69 &
\end{tabular}

\begin{tabular}{|c|c|c|c|c|c|c|c|}
\hline \multicolumn{8}{|c|}{ 8N/11W-14R1. Altitude, 2,313.7; depth, 164.9} \\
\hline $3-12-92$ & 86.41 & $6-17-92$ & 86.48 & $9-09-92$ & 86.59 & $12-14-92$ & 86.70 \\
\hline $4-04-92$ & 86.29 & 7-08-92 & 86.70 & $10-05-92$ & 86.72 & & \\
\hline $5-06-92$ & 86.48 & $8-05-92$ & 86.53 & $11-08-92$ & 86.52 & & \\
\hline
\end{tabular}

\begin{tabular}{|c|c|c|c|c|c|c|c|}
\hline \multicolumn{8}{|c|}{ 8N/11W-15Q1. Altitude, 2,304.3; depth, 177.8} \\
\hline $3-12-92$ & 78.55 & $6-17-92$ & 78.62 & $9-09-92$ & 78.61 & $12-14-92$ & 78.69 \\
\hline 4-04-92 & 78.43 & $7-08-92$ & 78.65 & $10-05-92$ & 78.69 & & \\
\hline $5-06-92$ & 78.54 & $8-05-92$ & 78.56 & $11-08-92$ & 78.55 & & \\
\hline \multicolumn{8}{|c|}{ 8N/12W-2Q1. Altitude, 2,283.8; depth, 72.7 } \\
\hline $4-06-92$ & 49.00 & $7-08-92$ & 49.68 & $9-09-92$ & 50.78 & $11-08-92$ & 52.13 \\
\hline $5-06-92$ & 48.94 & $8-05-92$ & 50.30 & $10-05-92$ & 51.29 & $12-14-92$ & 52.06 \\
\hline $6-17-92$ & 49.26 & & & & & & \\
\hline
\end{tabular}

\begin{tabular}{llllllll}
\hline \multicolumn{8}{c}{ 8N/12W-10J1. Altitude, 2,288.8; depth, 85 } \\
\hline $3-11-92$ & 36.43 & $5-06-92$ & 36.42 & $8-05-92$ & 36.88 & $11-08-92$ & 37.13 \\
$4-06-92$ & 36.33 & $6-17-92$ & 36.42 & $9-09-92$ & 36.92 & $12-14-92$ & 37.15 \\
$4-13-92$ & 36.48 & $7-08-92$ & 36.81 & $10-05-92$ & 37.03 & &
\end{tabular}

\begin{tabular}{|c|c|c|c|c|c|c|c|}
\hline \multicolumn{8}{|c|}{ Completed within the confining lacustrine unit in the Lancaster subbasin } \\
\hline \multicolumn{8}{|c|}{ 8N/9W-6D1. Altitude, 2,287.2; depth, 135.6} \\
\hline $1-09-92$ & 40.73 & 4-07-92 & 39.82 & $7-08-92$ & 42.61 & $10-05-92$ & 42.25 \\
\hline 2-04-92 & 40.83 & $5-09-92$ & 40.23 & $8-06-92$ & 42.44 & $11-07-92$ & 41.80 \\
\hline $3-10-92$ & 39.96 & $6-16-92$ & 40.61 & $9-09-92$ & 42.05 & $12-16-92$ & 41.22 \\
\hline \multicolumn{8}{|c|}{ 8N/10W-1Q4. Altitude, 2,301.7; depth, 130} \\
\hline $1-07-92$ & 52.94 & $4-04-92$ & 53.14 & $8-05-92$ & 53.32 & $11-07-92$ & 53.50 \\
\hline $1-13-92$ & 53.02 & $5-05-92$ & 53.19 & $9-09-92$ & 53.41 & $12-14-92$ & 53.56 \\
\hline $2-03-92$ & 53.02 & $6-09-92$ & 53.28 & $9-14-92$ & 53.43 & & \\
\hline 2-24-92 & 53.07 & $7-07-92$ & 53.16 & $10-05-92$ & 53.44 & & \\
\hline $3-10-92$ & 53.10 & $7-13-92$ & 53.22 & $10-29-92$ & 53.47 & & \\
\hline \multicolumn{8}{|c|}{ 8N/10W-4R5. Altitude, 2,301.4; depth, 150} \\
\hline $1-09-92$ & 89.17 & $4-05-92$ & 88.87 & $7-08-92$ & 89.11 & $10-05-92$ & 89.58 \\
\hline 2-03-92 & 89.11 & $5-07-92$ & 89.01 & $8-11-92$ & 89.25 & $11-08-92$ & 89.67 \\
\hline $3-09-92$ & 88.93 & $6-16-92$ & 89.14 & $9-09-92$ & 89.42 & $12-14-92$ & 89.57 \\
\hline
\end{tabular}


Table 2. Ground-water levels for wells and piezometers on Edwards Air Force Base, California, 1992--Continued

\begin{tabular}{|c|c|c|c|c|c|c|c|}
\hline Date & Water level & Date & Water level & Date & Water level & Date & Water level \\
\hline \multicolumn{8}{|c|}{ Completed within the confining lacustrine unit in the Lancaster subbasin--Continued } \\
\hline \multicolumn{8}{|c|}{ 8N/10W-4R6. Altitude, 2,301.4; depth, 100} \\
\hline $1-09-92$ & 59.41 & $4-05-92$ & 59.47 & $7-08-92$ & 58.35 & $10-05-92$ & 58.45 \\
\hline $2-03-92$ & 59.49 & $5-07-92$ & 59.52 & $8-11-92$ & 58.43 & $11-08-92$ & 58.46 \\
\hline $3-09-92$ & 59.49 & $6-16-92$ & 59.45 & $9-09-92$ & 58.44 & $12-14-92$ & 58.47 \\
\hline \multicolumn{8}{|c|}{ 8N/10W-5A6. Altitude, 2,287.3; depth, 55} \\
\hline $1-09-92$ & 28.36 & $3-31-92$ & 28.38 & $7-08-92$ & 28.49 & $10-05-92$ & 28.64 \\
\hline $2-03-92$ & 28.36 & $5-08-92$ & 28.39 & $8-11-92$ & 28.56 & $11-08-92$ & 28.66 \\
\hline $3-09-92$ & 28.36 & $6-16-92$ & 28.46 & $9-09-92$ & 28.62 & $12-14-92$ & 28.66 \\
\hline \multicolumn{8}{|c|}{ 9N/9W-28A5. Altitude, 2,271.1; depth, 65} \\
\hline $1-10-92$ & 35.27 & $3-11-92$ & 40.35 & $7-13-92$ & 40.67 & $10-06-92$ & 40.84 \\
\hline $1-17-92$ & 37.65 & $4-02-92$ & 40.45 & $7-30-92$ & 40.69 & $10-28-92$ & 40.67 \\
\hline $2-05-92$ & 39.69 & $5-05-92$ & 40.43 & $8-11-92$ & 40.70 & $12-17-92$ & 40.04 \\
\hline $2-21-92$ & 40.24 & $6-09-92$ & 40.60 & $9-09-92$ & 40.74 & & \\
\hline $3-09-92$ & 40.25 & $7-07-92$ & 40.69 & $9-14-92$ & 40.60 & & \\
\hline
\end{tabular}

\begin{tabular}{llllllll}
\hline \multicolumn{7}{c}{ 9N/10W-34R5. Altitude, 2,290.5; depth, 90 } \\
\hline $1-10-92$ & 17.45 & $5-08-92$ & 17.92 & $9-07-92$ & 18.33 & $12-15-92$ & 17.58 \\
$2-03-92$ & 17.41 & $6-16-92$ & 17.85 & $9-17-92$ & 18.26 & & \\
$3-09-92$ & 17.49 & $7-08-92$ & 17.99 & $10-05-92$ & 18.28 & \\
$4-05-92$ & 17.70 & $8-11-92$ & 18.21 & $10-29-92$ & 18.04 &
\end{tabular}

\begin{tabular}{|c|c|c|c|c|c|c|c|}
\hline \multicolumn{8}{|c|}{ 9N/10W-36J4. Altitude, 2,283.0; depth, 95} \\
\hline $1-09-92$ & 22.09 & $5-09-92$ & 21.80 & $8-06-92$ & 21.46 & $11-07-92$ & 21.63 \\
\hline $2-04-92$ & 21.84 & $6-16-92$ & 21.70 & 9-09-92 & 21.53 & $12-16-92$ & 21.81 \\
\hline $3-10-92$ & 21.77 & $7-08-92$ & 21.57 & $9-16-92$ & 21.53 & & \\
\hline $4-07-92$ & 21.70 & $7-31-92$ & 21.47 & $10-07-92$ & 21.57 & & \\
\hline \multicolumn{8}{|c|}{ 9N/10W-36P3. Altitude, 2,291.2; depth, 120} \\
\hline $1-09-92$ & 27.53 & $5-09-92$ & 27.01 & $7-29-92$ & 27.37 & $10-07-92$ & 27.27 \\
\hline $2-03-92$ & 27.55 & $6-16-92$ & 27.14 & $8-06-92$ & 27.37 & $10-30-92$ & 27.31 \\
\hline $3-10-92$ & 27.06 & $7-08-92$ & 27.36 & 9-09-92 & 27.31 & $12-15-92$ & 27.19 \\
\hline $4-07-92$ & 27.00 & $7-15-92$ & 27.35 & $9-15-92$ & 27.30 & & \\
\hline
\end{tabular}

\begin{tabular}{|c|c|c|c|c|c|c|c|}
\hline \multicolumn{8}{|c|}{ Completed in the small, unconfined, unnamed subbasin } \\
\hline \multicolumn{8}{|c|}{ 9N/10W-8P1. Altitude, 2,370.5; depth, 132.6} \\
\hline $1-09-92$ & 81.58 & $4-05-92$ & 81.47 & $7-08-92$ & 81.54 & $10-06-92$ & 81.45 \\
\hline $2-04-92$ & 81.53 & $5-11-92$ & 81.53 & $8-10-92$ & 81.42 & $11-07-92$ & 81.46 \\
\hline $3-10-92$ & 81.56 & $6-17-92$ & 81.50 & $9-08-92$ & 81.50 & $12-15-92$ & 81.41 \\
\hline \multicolumn{8}{|c|}{ 9N/10W-16F1. Altitude, 2,320.7; depth, 458} \\
\hline $1-08-92$ & 111.53 & $4-05-92$ & 111.33 & $7-07-92$ & 110.40 & $10-06-92$ & 110.92 \\
\hline 2-04-92 & 111.49 & $5-11-92$ & 111.30 & $8-10-92$ & 110.98 & $11-08-92$ & 110.80 \\
\hline $3-10-92$ & 111.40 & $6-16-92$ & 111.16 & $9-07-92$ & 111.02 & $12-15-92$ & 110.74 \\
\hline \multicolumn{8}{|c|}{ 9N/10W-16L1. Altitude, 2,319.5; depth, 500} \\
\hline $1-08-92$ & 117.66 & $4-05-92$ & 116.29 & $7-07-92$ & 118.49 & $10-06-92$ & 116.95 \\
\hline 2-04-92 & 116.98 & $5-11-92$ & 121.00 & $8-10-92$ & 117.74 & $11-08-92$ & 116.37 \\
\hline $3-10-92$ & 116.61 & $6-17-92$ & 121.11 & $9-08-92$ & 117.70 & $12-14-92$ & 116.20 \\
\hline
\end{tabular}


Table 2. Ground-water levels for wells and piezometers on Edwards Air Force Base, California, 1992--Continued

\begin{tabular}{|c|c|c|c|c|c|c|c|}
\hline Date & Water level & Date & Water level & Date & Water level & Date & Water level \\
\hline \multicolumn{8}{|c|}{ Completed in the small, unconfined, unnamed subbasin--Continued } \\
\hline \multicolumn{8}{|c|}{ 9N/10W-16L2. Altitude, 2,319.0; depth, 732} \\
\hline $1-08-92$ & 113.40 & $4-05-92$ & 112.16 & $7-07-92$ & 113.65 & $10-06-92$ & 112.96 \\
\hline $2-04-92$ & 112.87 & 5-11-92 & 112.43 & $8-10-92$ & 113.08 & $11-08-92$ & 112.90 \\
\hline $3-10-92$ & 112.42 & $6-16-92$ & 113.46 & $9-07-92$ & 113.06 & $12-14-92$ & 112.92 \\
\hline \multicolumn{8}{|c|}{ 9N/10W-16L3. Altitude, 2,318.7; depth, 270} \\
\hline $1-07-92$ & 113.06 & $4-05-92$ & 112.84 & $7-07-92$ & 111.53 & $10-06-92$ & 112.64 \\
\hline $2-04-92$ & 113.06 & $5-11-92$ & 112.85 & $8-10-92$ & 112.07 & $11-08-92$ & 112.72 \\
\hline $3-10-92$ & 112.91 & $6-16-92$ & 112.87 & $9-07-92$ & 112.58 & $12-14-92$ & 112.69 \\
\hline \multicolumn{8}{|c|}{ 9N/10W-16M1. Altitude, 2,324.0; depth, 140.5} \\
\hline $1-09-92$ & 120.25 & $4-05-92$ & 120.33 & $7-07-92$ & 120.45 & $10-06-92$ & 120.58 \\
\hline $2-04-92$ & 120.29 & $5-11-92$ & 120.35 & $8-10-92$ & 120.50 & $11-08-92$ & 120.58 \\
\hline $3-10-92$ & 120.33 & $6-16-92$ & 120.40 & $9-07-92$ & 120.58 & $12-15-92$ & 120.60 \\
\hline \multicolumn{8}{|c|}{ 9N/10W-16N1. Altitude, 2,325.8; depth, 376} \\
\hline $1-08-92$ & 122.04 & $4-05-92$ & 122.06 & $7-07-92$ & 122.22 & $10-06-92$ & 122.29 \\
\hline $2-04-92$ & 122.07 & $5-11-92$ & 122.11 & $8-10-92$ & 122.21 & $11-08-92$ & 122.25 \\
\hline $3-10-92$ & 122.07 & $6-16-92$ & 122.12 & $9-07-92$ & 122.29 & $12-15-92$ & 122.32 \\
\hline \multicolumn{8}{|c|}{ 9N/10W-16P1. Altitude, 2,320.2; depth, 532} \\
\hline $1-08-92$ & 118.07 & $4-05-92$ & 116.88 & $7-07-92$ & 118.75 & $10-06-92$ & 117.48 \\
\hline $2-04-92$ & 117.50 & $5-11-92$ & 121.33 & $8-10-92$ & 118.17 & $11-08-92$ & 116.96 \\
\hline $3-10-92$ & 117.16 & $6-16-92$ & 121.00 & $9-07-92$ & 118.11 & $12-15-92$ & 116.81 \\
\hline \multicolumn{8}{|c|}{ 9N/10W-16R1. Altitude, 2,312.9; depth, 840} \\
\hline $1-08-92$ & 100.45 & $3-30-92$ & 100.40 & $7-14-92$ & 100.00 & $10-06-92$ & 103.73 \\
\hline $1-28-92$ & 100.46 & $5-05-92$ & 100.66 & $8-10-92$ & 101.79 & $10-29-92$ & 103.26 \\
\hline $2-04-92$ & 100.48 & $6-16-92$ & 100.69 & $9-07-92$ & 103.55 & $11-08-92$ & 103.11 \\
\hline $3-10-92$ & 100.47 & $7-07-92$ & 99.71 & $9-15-92$ & 103.53 & $12-15-92$ & 102.94 \\
\hline \multicolumn{8}{|c|}{ 9N/10W-16R2. Altitude, 2,312.8; depth 584} \\
\hline $1-08-92$ & 101.41 & $3-30-92$ & 101.48 & $7-14-92$ & 101.56 & $10-06-92$ & 104.68 \\
\hline $1-28-92$ & 101.44 & $5-05-92$ & 101.59 & $8-10-92$ & 103.45 & $10-29-92$ & 104.19 \\
\hline $2-04-92$ & 101.48 & $6-11-92$ & 101.62 & $9-07-92$ & 104.98 & $12-15-92$ & 104.14 \\
\hline $3-10-92$ & 101.53 & $7-07-92$ & 101.46 & $9-15-92$ & 104.74 & & \\
\hline \multicolumn{8}{|c|}{ 9N/10W-16R3. Altitude, 2,312.8; depth, 360} \\
\hline $1-08-92$ & 101.69 & $3-30-92$ & 101.77 & $7-14-92$ & 102.25 & $10-06-92$ & 104.29 \\
\hline $1-28-92$ & 101.68 & $5-05-92$ & 101.84 & $8-10-92$ & 103.28 & $10-29-92$ & 104.19 \\
\hline $2-04-92$ & 101.77 & $6-16-92$ & 101.86 & $9-07-92$ & 104.47 & $11-08-92$ & 104.16 \\
\hline $3-10-92$ & 101.77 & $7-07-92$ & 102.27 & $9-15-92$ & 104.53 & $12-15-92$ & 104.32 \\
\hline \multicolumn{8}{|c|}{ 9N/10W-16R4. Altitude, 2,308.4; depth, 700 } \\
\hline $2-04-92$ & 99.74 & $5-11-92$ & 99.91 & $8-10-92$ & $136.69^{1}$ & $11-08-92$ & 103.03 \\
\hline $3-10-92$ & 99.80 & $6-16-92$ & 99.91 & $9-07-92$ & $132.89^{1}$ & $12-15-92$ & 103.10 \\
\hline $4-05-92$ & 99.85 & $7-07-92$ & $116.09^{1}$ & $10-06-92$ & 102.79 & & \\
\hline
\end{tabular}

Footnote at end of table. 
Table 2. Ground-water levels for wells and piezometers on Edwards Air Force Base, California, 1992--Continued

\begin{tabular}{|c|c|c|c|c|c|c|c|}
\hline Date & Water level & Date & Water level & Date & Water level & Date & Water level \\
\hline \multicolumn{8}{|c|}{ Completed near the ground-water divide on Rogers Lake } \\
\hline \multicolumn{8}{|c|}{ 10N/9W-27C1. Altitude, 2,272.4, depth 222} \\
\hline $2-05-92$ & 79.03 & $5-11-82$ & 79.30 & $8-07-92$ & 79.50 & $11-07-92$ & 79.76 \\
\hline $3-11-92$ & 80.18 & $6-17-92$ & 79.42 & $9-09-92$ & 79.60 & $12-16-92$ & 79.89 \\
\hline $4-05-92$ & 79.14 & $7-07-92$ & 79.35 & $10-06-92$ & 79.69 & & \\
\hline \multicolumn{8}{|c|}{ 10N/9W-27C2. Altitude, 2,272.4; depth, 160} \\
\hline $2-05-92$ & 78.90 & $5-11-92$ & 79.18 & $8-06-92$ & 79.36 & $11-07-92$ & 79.61 \\
\hline $3-11-92$ & 79.07 & $6-17-92$ & 79.28 & $9-09-92$ & 79.45 & $12-16-92$ & 79.78 \\
\hline $4-05-92$ & 79.11 & $7-07-92$ & 79.23 & $10-06-92$ & 79.61 & & \\
\hline \multicolumn{8}{|c|}{ 10N/9W-27C3. Altitude, 2,272.4; depth, 80} \\
\hline $2-05-92$ & 74.38 & $5-11-92$ & 74.42 & $8-07-92$ & 74.45 & $11-07-92$ & 74.40 \\
\hline $3-11-92$ & 75.39 & $6-17-92$ & 74.42 & $9-09-92$ & 74.51 & $12-16-92$ & 74.41 \\
\hline $4-05-92$ & 74.44 & $7-07-92$ & 74.44 & $10-06-92$ & 74.49 & & \\
\hline \multicolumn{8}{|c|}{ Completed in the unconfined aquifer in the North Muroc subbasin } \\
\hline \multicolumn{8}{|c|}{ 10N/9W-4D1. Altitude, 2,304.2; depth, 456.2} \\
\hline $2-06-92$ & 127.11 & $5-09-92$ & 127.66 & $8-07-92$ & 129.12 & $11-07-92$ & 128.61 \\
\hline $3-11-92$ & 126.88 & $6-17-92$ & 128.20 & $9-10-92$ & 129.54 & $12-16-92$ & 128.19 \\
\hline 4-05-92 & 127.20 & $7-09-92$ & 128.75 & $10-06-92$ & 129.43 & & \\
\hline \multicolumn{8}{|c|}{ 10N/9W-10B1. Altitude, 2,278.6; depth, 312} \\
\hline $1-08-92$ & 95.70 & $4-05-92$ & 95.80 & $7-09-92$ & 95.91 & $10-06-92$ & 96.06 \\
\hline $2-05-92$ & 95.69 & $5-09-92$ & 95.80 & $8-07-92$ & 95.95 & $11-07-92$ & 96.11 \\
\hline $3-11-92$ & 95.72 & $6-17-92$ & 95.88 & $9-10-92$ & 96.06 & $12-17-92$ & 96.18 \\
\hline \multicolumn{8}{|c|}{ 10N/9W-10B2. Altitude, 2,278.6; depth, 150} \\
\hline $1-08-92$ & 95.52 & $4-05-92$ & 95.61 & $7-09-92$ & 95.72 & $10-06-92$ & 95.88 \\
\hline $2-05-92$ & 95.48 & $5-09-92$ & 95.60 & $8-07-92$ & 95.77 & $11-07-92$ & 95.91 \\
\hline $3-11-92$ & 95.51 & $6-17-92$ & 95.67 & $9-10-92$ & 95.88 & $12-17-92$ & 96.01 \\
\hline \multicolumn{8}{|c|}{ 10N/9W-24A2. Altitude, 2,290.6; depth, 278.7 } \\
\hline $1-08-92$ & 94.51 & $4-05-92$ & 94.63 & $7-09-92$ & 94.78 & $10-06-92$ & 95.04 \\
\hline $2-05-92$ & 94.49 & $5-09-92$ & 94.65 & $8-07-92$ & 94.85 & $11-07-92$ & 94.98 \\
\hline $3-11-92$ & 94.54 & $6-17-92$ & 94.73 & $9-10-92$ & 94.99 & $12-16-92$ & 95.24 \\
\hline \multicolumn{8}{|c|}{ 11N/9W-32Q1. Altitude, 2,302.9; depth, 450} \\
\hline 1-07-92 & 127.25 & $4-05-92$ & 127.72 & $7-09-92$ & 130.06 & $10-06-92$ & 130.46 \\
\hline 2-06-92 & 127.35 & $5-09-92$ & 128.27 & $8-07-92$ & 130.64 & $11-07-92$ & 129.00 \\
\hline 3-11-92 & 126.88 & $6-17-92$ & 128.95 & $9-10-92$ & 130.99 & $12-16-92$ & 128.44 \\
\hline \multicolumn{8}{|c|}{ 11N/9W-36R1. Altitude, 2,311.9; depth, 254.1} \\
\hline $2-06-92$ & 121.80 & $5-09-92$ & 121.98 & $8-07-92$ & 122.05 & $11-07-92$ & 122.20 \\
\hline $3-11-92$ & 121.82 & $6-17-92$ & 121.98 & $9-10-92$ & 122.19 & $12-17-92$ & 122.27 \\
\hline 4-04-92 & 121.98 & 7-09-92 & 122.06 & $10-06-92$ & 122.18 & & \\
\hline
\end{tabular}

${ }^{1}$ Pumping level (or well pumping). 
A. Piezometers $8 \mathrm{~N} / 10 \mathrm{~W}-101,-102,-103$, and -104 (south of the South Track well field)

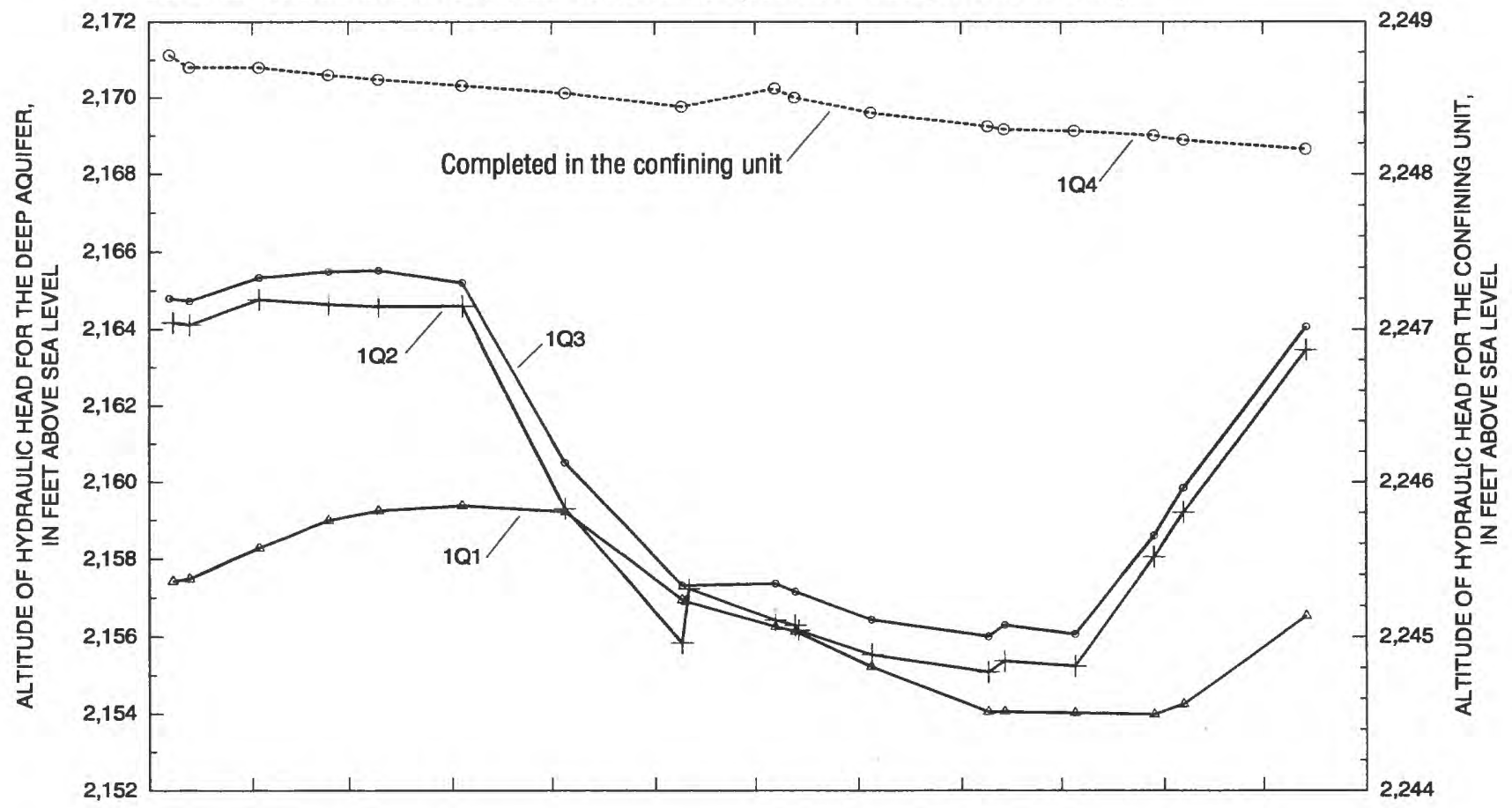

B. Piezometers 8N/10W-4R1, -4R2, -4R3, -4R4, -4R5 and -4R6 (southwest of the Branch Park well field)

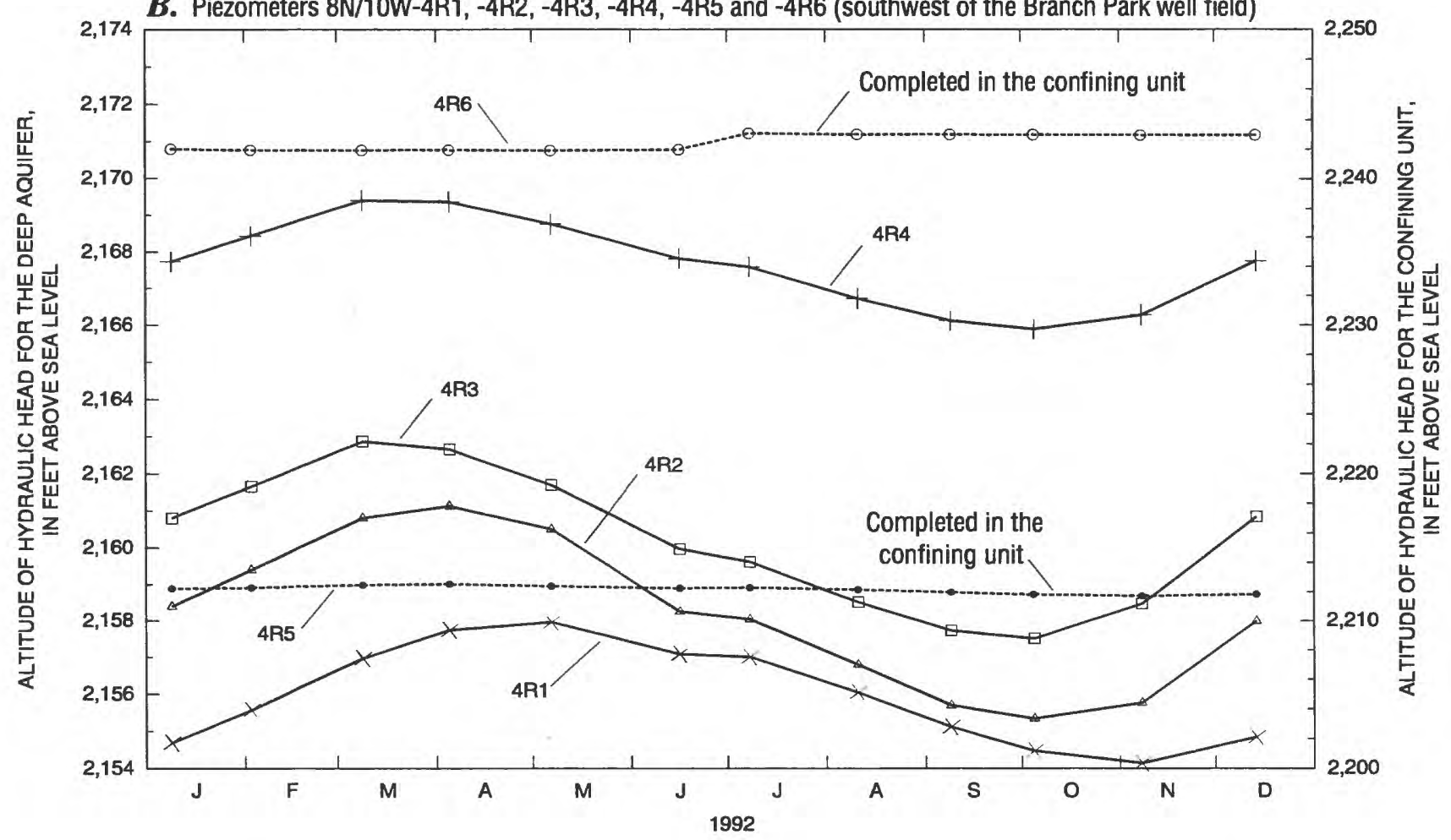

Figure 6. Hydraulic heads for U.S. Geological Survey piezometers on Edwards Air Force Base, California, 1992. (Screened in the deep aquifer of the Lancaster subbasin except where indicated. See figure 5 for piezometer locations.) 

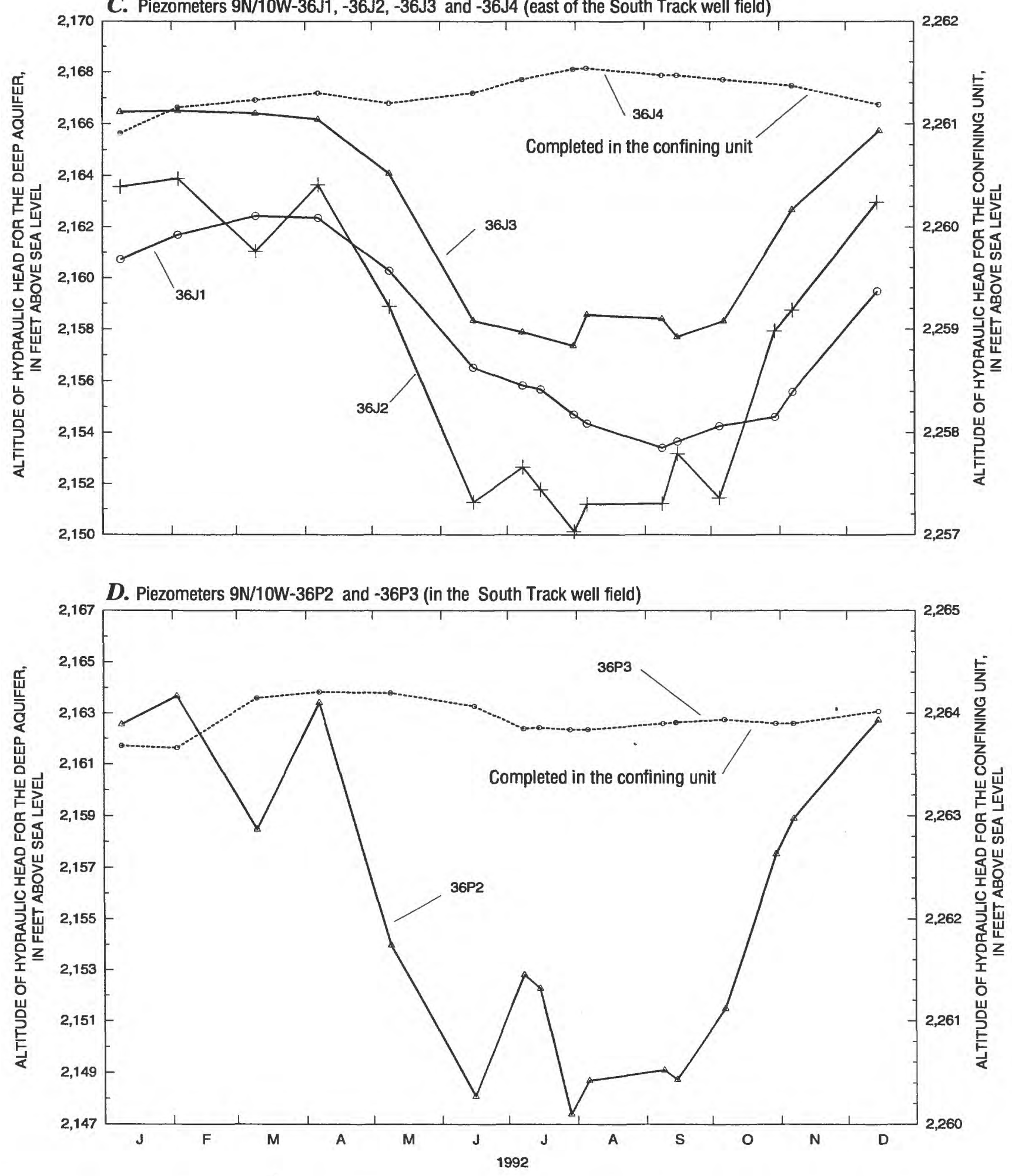

Figure 6. Hydraulic heads for U.S. Geological Survey piezometers on Edwards Air Force Base, California, 1992--Continued. 
$\boldsymbol{E}$. Piezometers 9N/10W-25P1 and -25P2 (north of South Track well field)

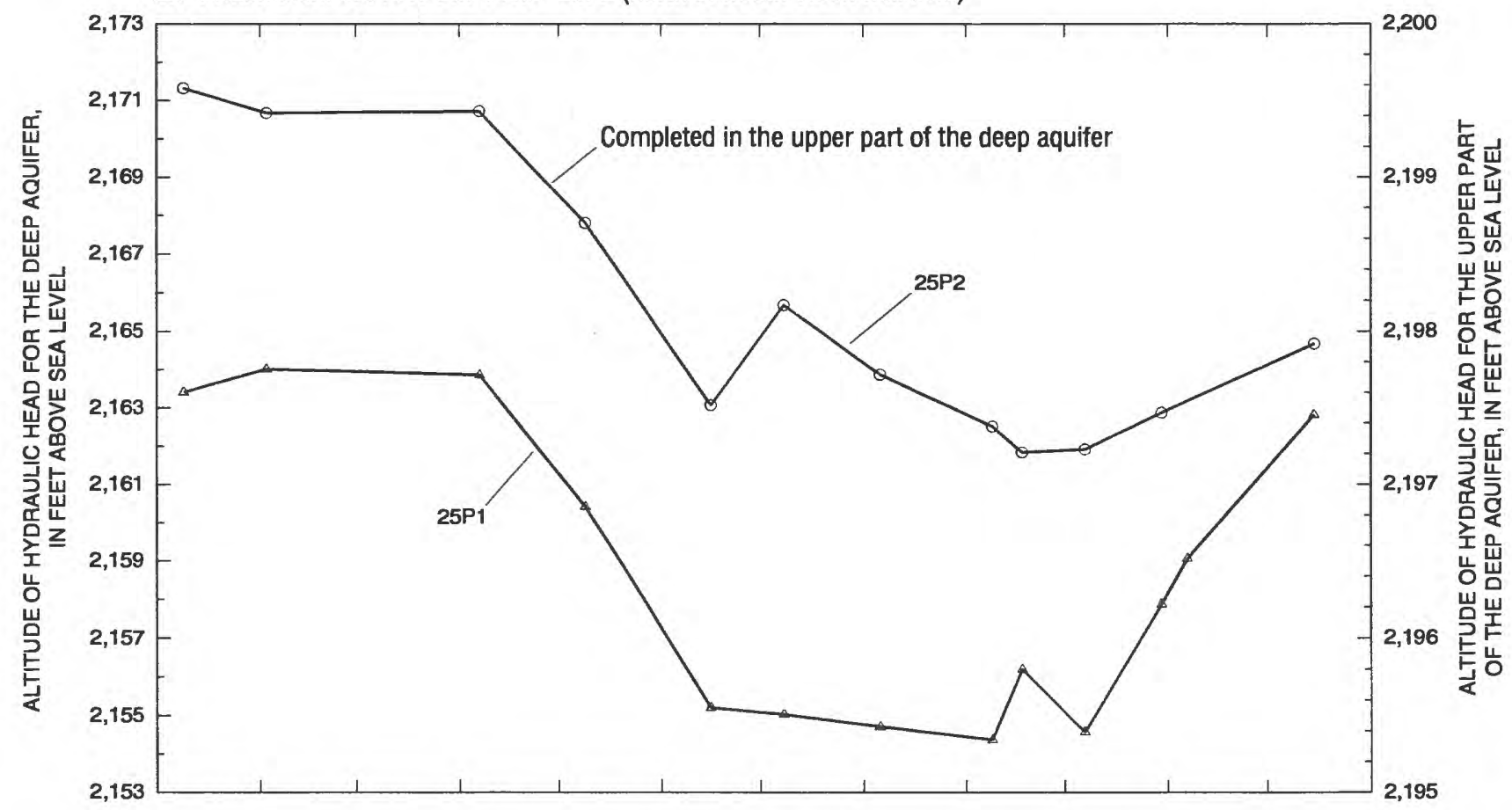

F. Piezometers 9N/10W-34R2, -34R3, -34R4, and -34R5 (east of Branch Park well field)

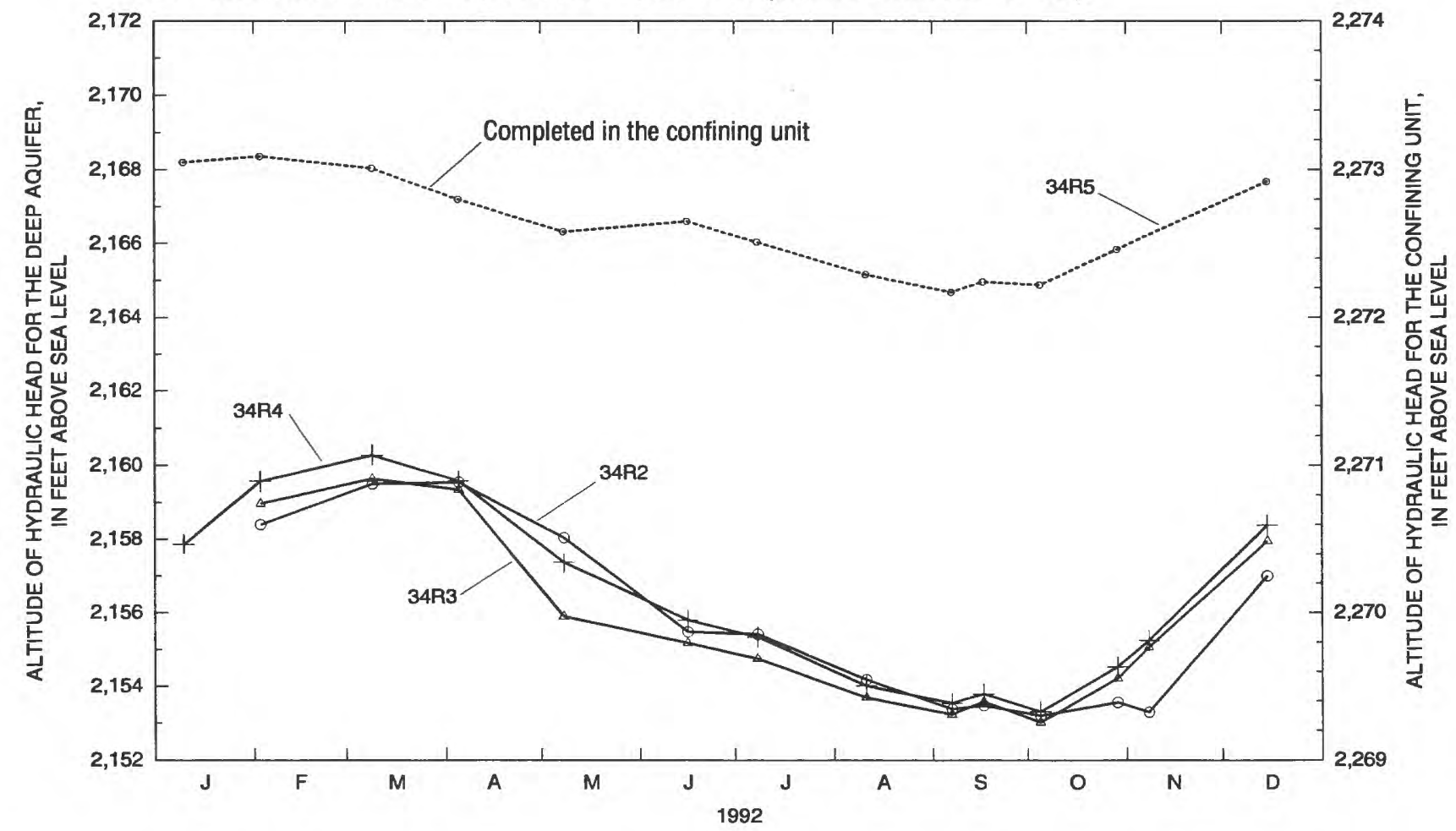

Figure 6. Hydraulic heads for U.S. Geological Survey piezometers on Edwards Air Force Base, California, 1992--Continued. 
G. Piezometers $8 \mathrm{~N} / 10 \mathrm{~W}-5 \mathrm{A1},-5 \mathrm{~A} 2,-5 \mathrm{A3},-5 \mathrm{A4}$, and $-5 \mathrm{A6}$ (west of Branch Park well field)

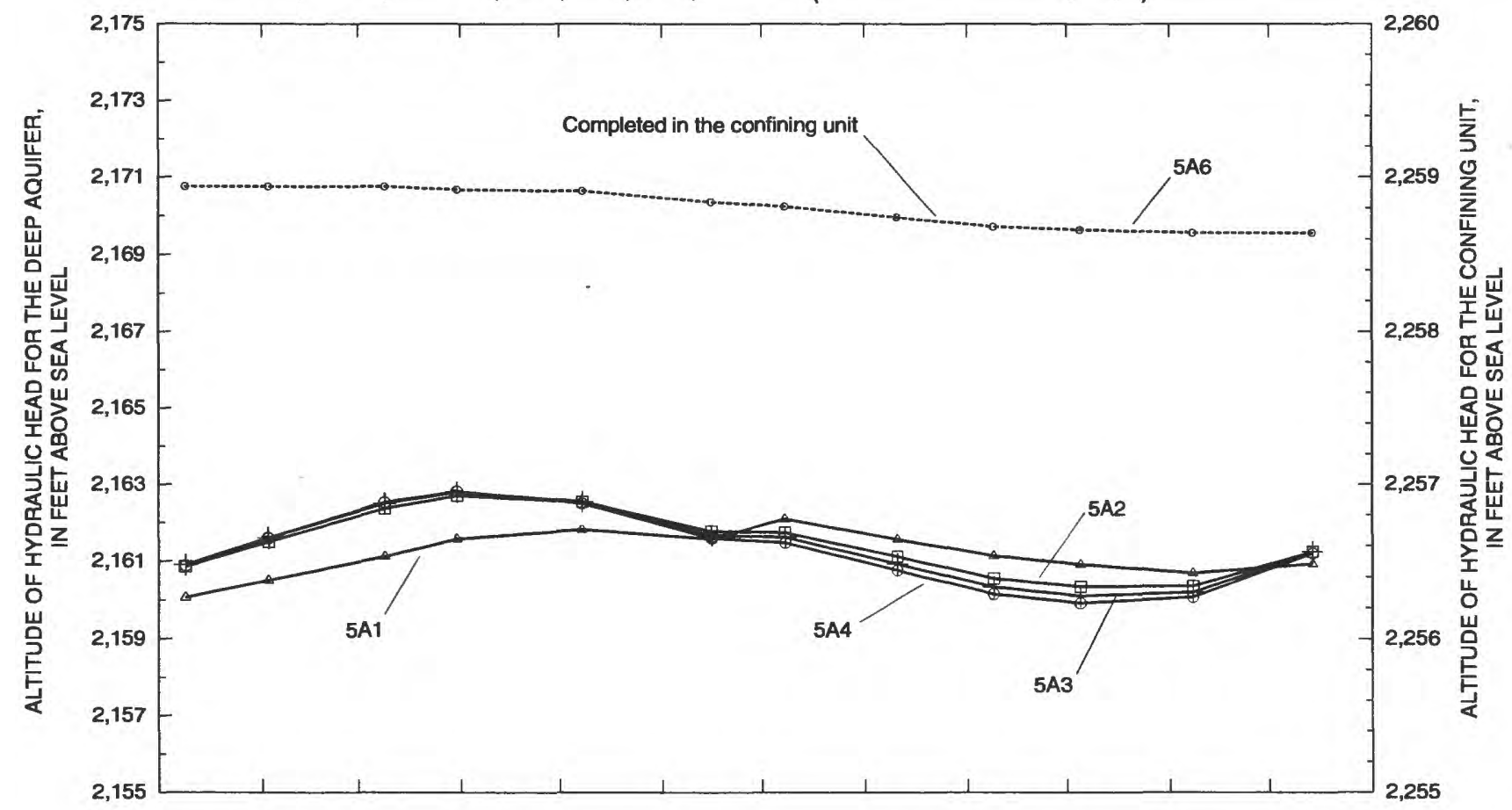

H. Piezometers 9N/10W-28H3 and -28H4 (northwest of Branch Park well field)

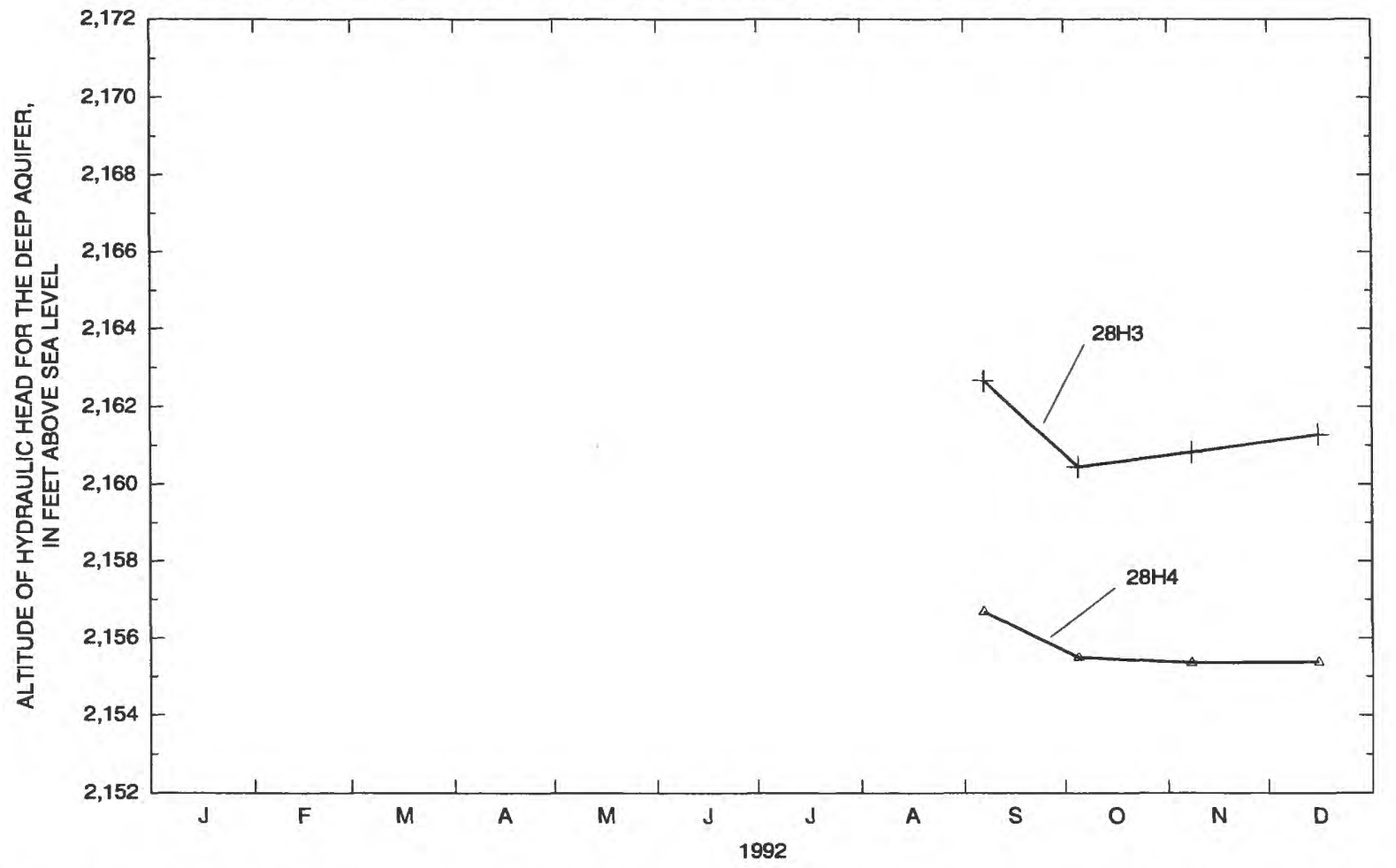

Figure 6. Hydraulic heads for U.S. Geological Survey piezometers on Edwards Air Force Base, California, 1992--Continued. 
I. Piezometers 9N/10W-27P1, -27P2, and -27P3 (north of Branch Park well field)
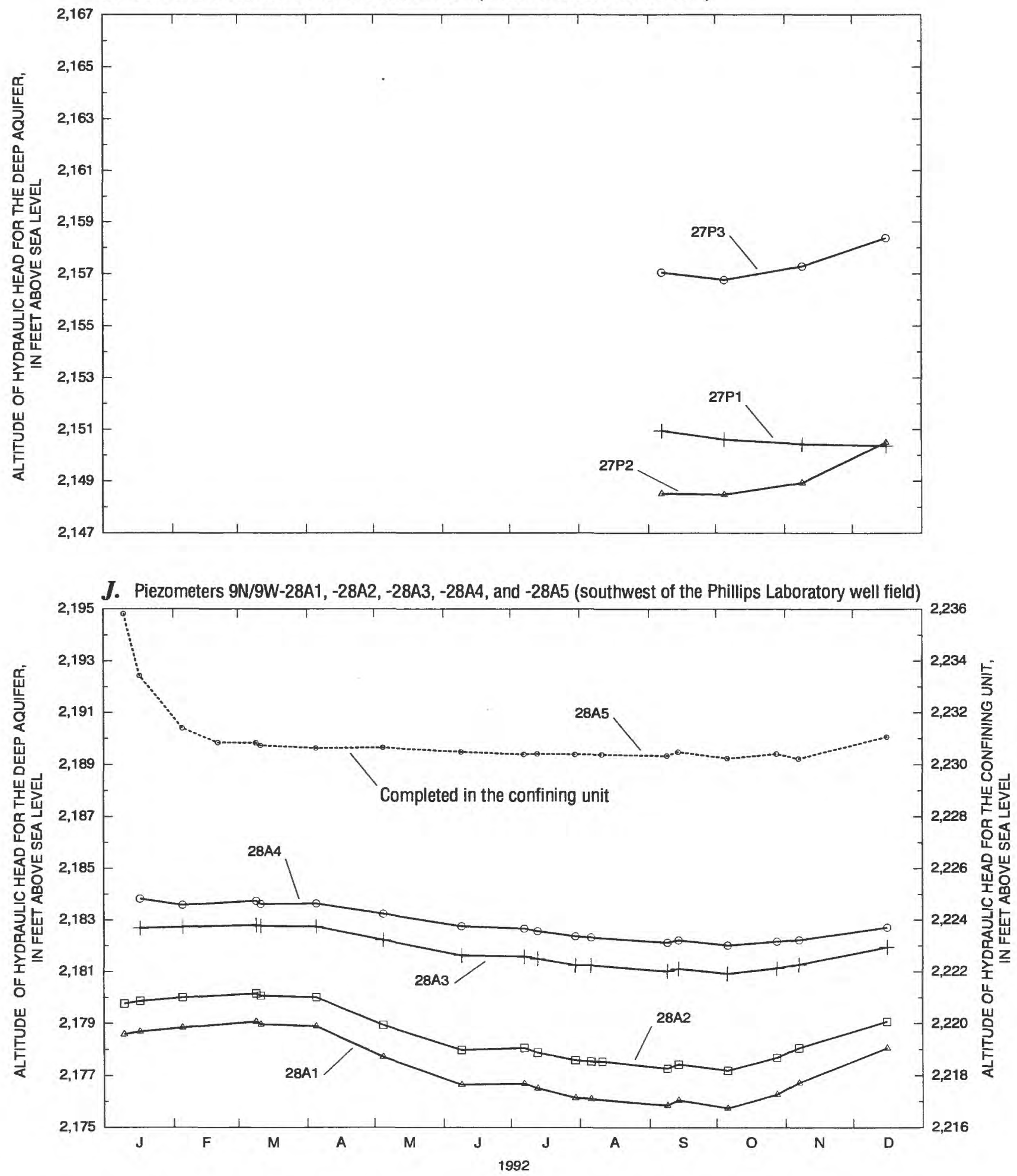

Figure 6. Hydraulic heads for U.S. Geological Survey piezometers on Edwards Air Force Base, California, 1992--Continued. 


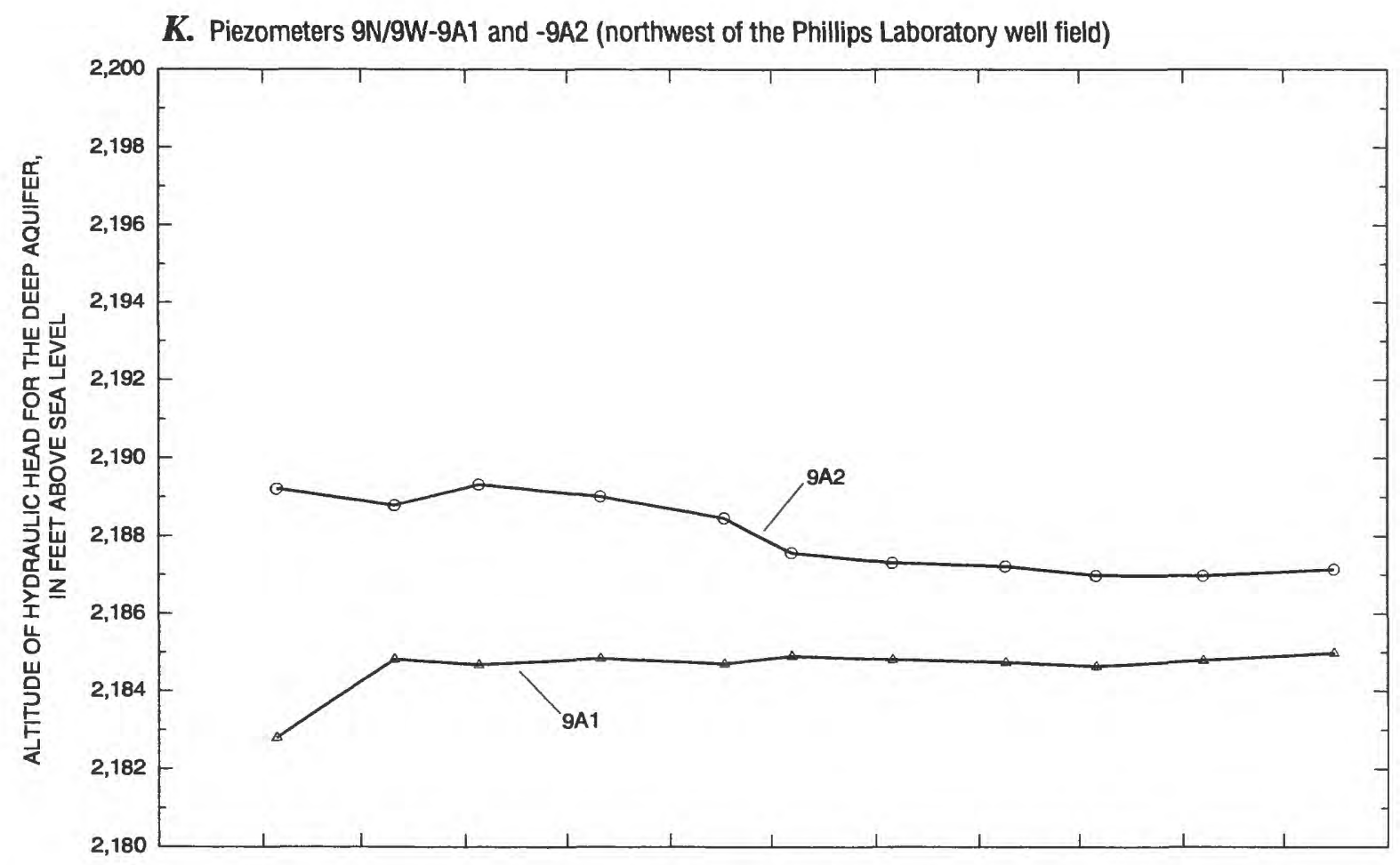

L. Piezometers $10 \mathrm{~N} / 9 \mathrm{~W}-27 \mathrm{C} 1,-27 \mathrm{C} 2$, and $-27 \mathrm{C3}$ (north of the Phillips Laboratory well field near the ground-water divide)

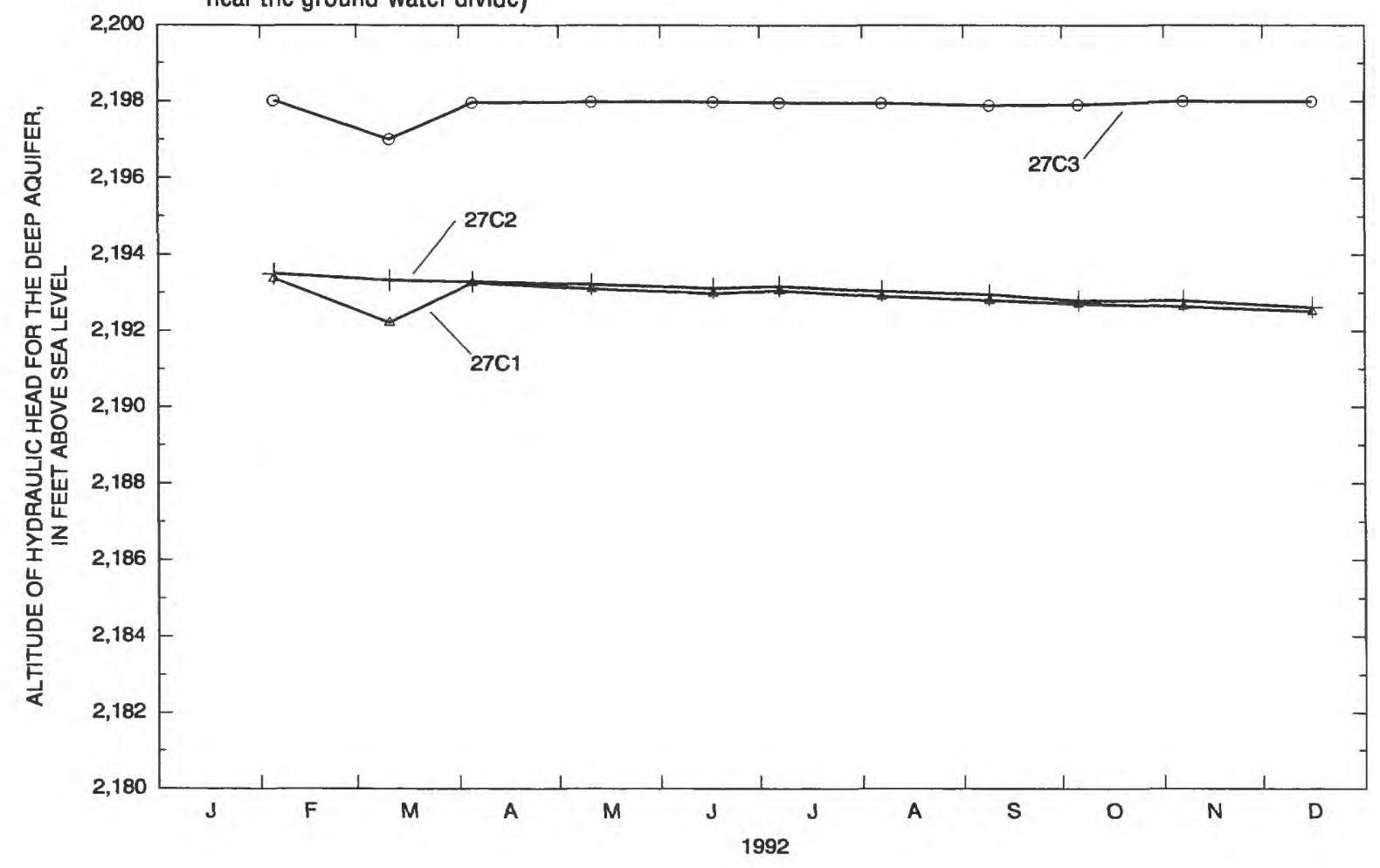

Figure 6. Hydraulic heads for U.S. Geological Survey piezometers on Edwards Air Force Base, California, 1992--Continued. 
M. Piezometers 10N/9W-10B1 and -10B2 (southeast of the North Base well field)

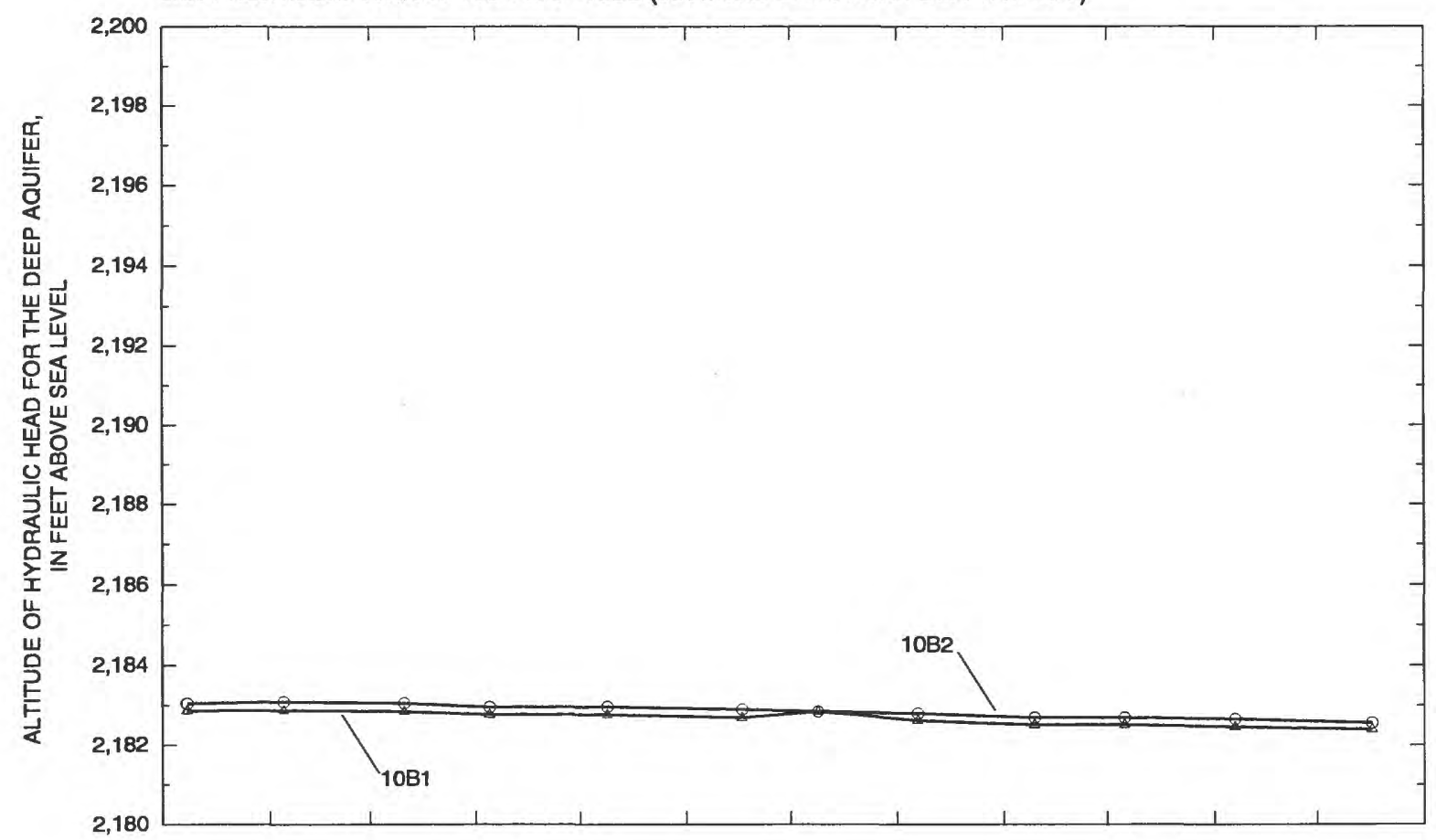

N. Piezometers 9N/10W-16F1 (north of Graham Ranch well field) and 9N/10W-16R1, -16R2, and -16R3 (in the Graham Ranch well field)

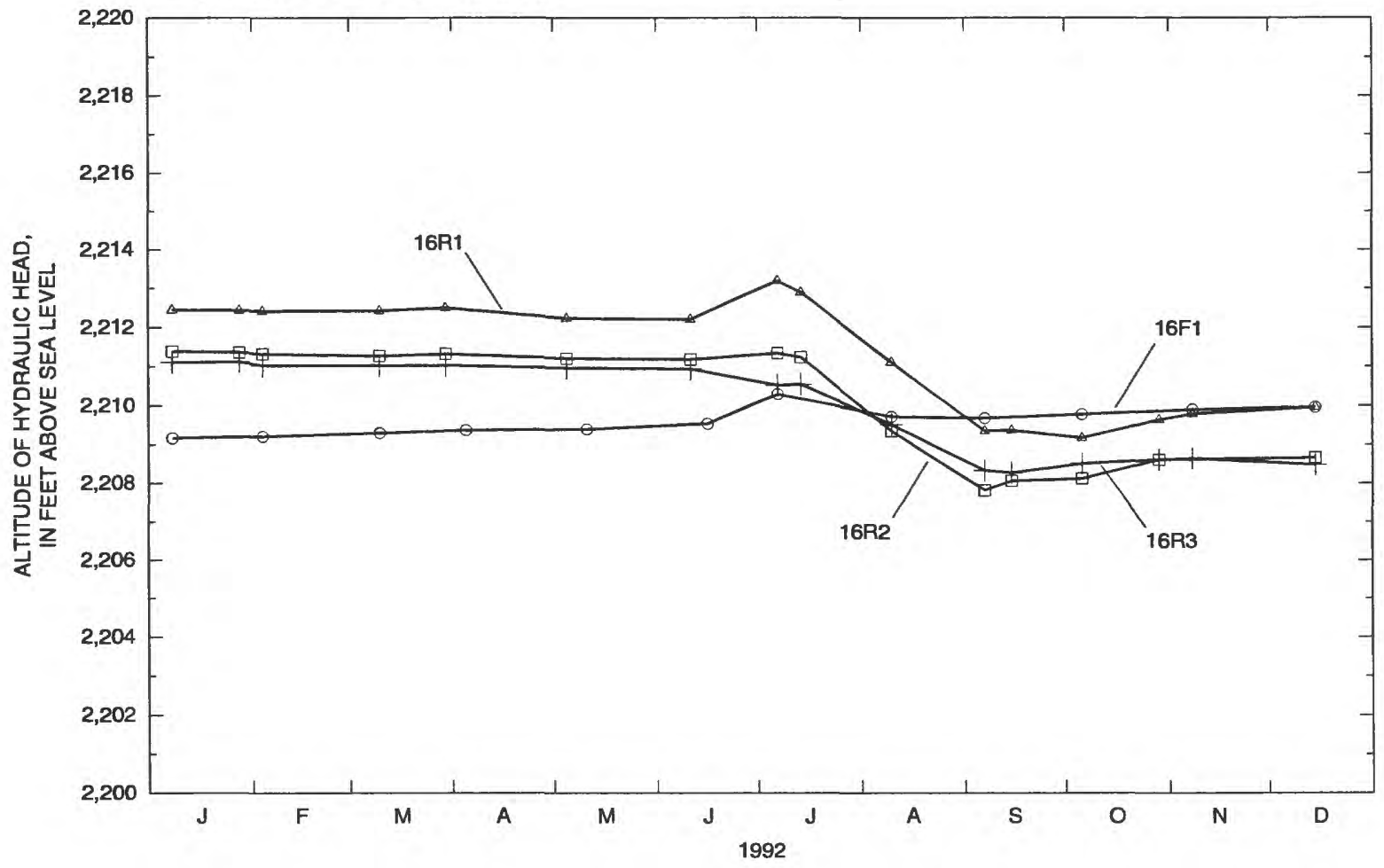

Figure 6. Hydraulic heads for U.S. Geological Survey piezometers on Edwards Air Force Base, California, 1992--Continued. 
aquifer near the South Track well field (fig. 5) range from about 5 to $15 \mathrm{ft}$ between spring recovery and late summer drawdown levels (figs. $6 A$ through $6 N$ ).

Heads in piezometers $8 \mathrm{~N} / 10 \mathrm{~W}-1 \mathrm{Q} 1$ through $-1 \mathrm{Q} 3$ and $-4 \mathrm{R} 1$ through $-4 \mathrm{R} 4,9 \mathrm{~N} / 10 \mathrm{~W}-36 \mathrm{~J} 1$ through $-36 \mathrm{~J} 3$, $36 \mathrm{P} 2$, and $-34 \mathrm{R} 2$ through $-34 \mathrm{R} 4$ (figs. $6 A, 6 B, 6 C, 6 D$, and $6 F$ ) are higher than the lower contact of the confining unit (Londquist and others, 1993; Rewis, 1993) indicating confined, nonflowing artesian conditions west, south, and east of the South Track well field. Heads in piezometers 9N/10W-25P1 and -25P2 and 9N/9W-28A1 through -28A4 (figs. $6 E$ and $6 J$ ) are lower than the lower contact of the confining unit indicating unconfined aquifer conditions north of the well field.

On the basis of ground-water-level, lithologic, water-quality, and borehole-resistivity data for piezometer 8N/10W-1Q1, Londquist and others (1993, p. 66) suggest that there is a poor hydraulic connection between the upper and lower confined zones of the deep aquifer at that site. This poor connection may be due to consolidation of the deeper alluvium. Heads in piezometers $8 \mathrm{~N} / 10 \mathrm{~W}-4 \mathrm{R} 1,9 \mathrm{~N} / 10 \mathrm{~W}-36 \mathrm{~J} 1,9 \mathrm{~N} / 10 \mathrm{~W}$ $34 \mathrm{R} 2$, and $9 \mathrm{~N} / 10 \mathrm{~W}-5 \mathrm{~A} 1$ (figs. $6 B, 6 C, 6 F$, and $6 G$ ) which are completed below $1,500 \mathrm{ft}$ above sea level in the deep aquifer have similar delays in response to seasonal recharge and discharge stresses as for piezometer 8N/10W-1Q1 (fig. 6A). This similarity indicates that these piezometers may be completed in the lower confined zone.

Piezometers 8N/10W-4R5, -4R6, -5A6, 9N/9W28A5, 9N/10W-34R5, -36J4, and -36P3 were completed in the confining unit (table 1 , figs. $6 B-6 D, 6 F$, $6 G$, and $6 J$ ). Heads in piezometers $8 \mathrm{~N} / 10 \mathrm{~W}-4 \mathrm{R} 5$, 4R6, and 9N/9W-28A5 indicate little or no head change from April to September (figs. $6 B$ and $6 J$ ). Heads in piezometers $8 \mathrm{~N} / 10 \mathrm{~W}-1 \mathrm{Q} 4,-5 \mathrm{~A} 6,9 \mathrm{~N} / 10 \mathrm{~W}$ $34 \mathrm{R} 5$, and $-36 \mathrm{P} 3$ declined about $0.3,0.2,0.6$, and $0.3 \mathrm{ft}$, respectively, from April to September (table 2, figs. $6 A, 6 G, 6 F$, and $6 D$ ). Except for piezometer $8 \mathrm{~N} /$ 10W-1Q4 (fig. 6A), these declines correspond to declines in heads in the piezometers completed in the deep aquifer at these sites. Head in piezometer $9 \mathrm{~N} /$ $10 \mathrm{~W}-36 \mathrm{~J} 4$ increased about $0.2 \mathrm{ft}$ from April to September (table 2, fig. $6 \mathrm{C}$ ). The cause for this increase in head is not known, but may be due to underflow from a nearby dry wash or possibly leakage from water-supply lines in the area.
Piezometer 8N/10W-1Q4 was completed partly in the confining unit and partly in the overlying alluvium (tables 1 and 4) (Londquist and others, 1993). Hydraulic head in this piezometer was about $2,249 \mathrm{ft}$ above sea level in January 1992 and is representative of the hydraulic head in the principal aquifer (fig. $6 \mathrm{~A}$; table 2). The occurrence of the thin (approximately 40-foot thick) principal aquifer at this site indicates a possibility that the principal aquifer extends into the South Track well-field area. About $3 \mathrm{mi}$ west of well $8 \mathrm{~N} / 10 \mathrm{~W}-1 \mathrm{Q} 4$ at wells $8 \mathrm{~N} / 10 \mathrm{~W}-4 \mathrm{R} 1$ through $-4 \mathrm{R} 6$, the top of the confining unit is about $20 \mathrm{ft}$ higher than the hydraulic head in well -1Q4 (Londquist and others, 1993; Rewis, 1993) indicating a discontinuity of the principal aquifer in this part of the Lancaster subbasin.

The June 28, 1992, Landers and Big Bear earthquakes caused a static strain step (compressional) at EAFB as interpreted from hourly ground-water-level data recorded for piezometers $8 \mathrm{~N} / 10 \mathrm{~W}-1 \mathrm{Q} 2,9 \mathrm{~N} / 9 \mathrm{~W}$ 28A5, and 9N/10W-16R2 (D.L. Galloway, U.S. Geological Survey, written commun., 1992; Galloway, 1993; E. Roeloffs, U.S. Geological Survey, written commun., 1994). This strain step resulted in a volume compression of the aquifer materials, which caused an abrupt rise in hydraulic heads throughout the aquifer system. Heads recovered to near their pre-earthquake levels in the subsequent days or weeks. In some cases, strong pumping influences in the aquifer overwhelmed the aquifer response to the static strain step. The rise in heads between June and July recorded for piezometers $8 \mathrm{~N} / 10 \mathrm{~W}-1 \mathrm{Q} 4,8 \mathrm{~N} / 10 \mathrm{~W}-4 \mathrm{R} 6,9 \mathrm{~N} / 10 \mathrm{~W}-25 \mathrm{P} 2,8 \mathrm{~N} / 10 \mathrm{~W}-$ 5A1, 9N/9W-9A1, -28A1, -28A2, 10N/9W-27C1, $27 \mathrm{C} 2,9 \mathrm{~N} / 10 \mathrm{~W}-16 \mathrm{~F} 1,-16 \mathrm{R} 1$, and $-16 \mathrm{R} 2$ (figs. $6 A, 6 B$, $6 E, 6 G, 6 J, 6 K, 6 L$, and $6 N$ ) may reflect the same abrupt rise and subsequent decline response as those reported by Galloway (1993) and E. Roeloffs (U.S. Geological Survey, written commun., 1994).

\section{Pumpage and Hydraulic Heads in Production Well Fields}

Seven production well fields (fig. 5) provide potable and nonpotable water at EAFB. South Track, South Base, and North Base well fields provide potable water to the main facilities of the base. Branch Park and Graham Ranch well fields provide nonpotable water for landscape and recreational uses. The two Phillips Laboratory well fields supply potable ground water for potable and nonpotable uses for that facility. 


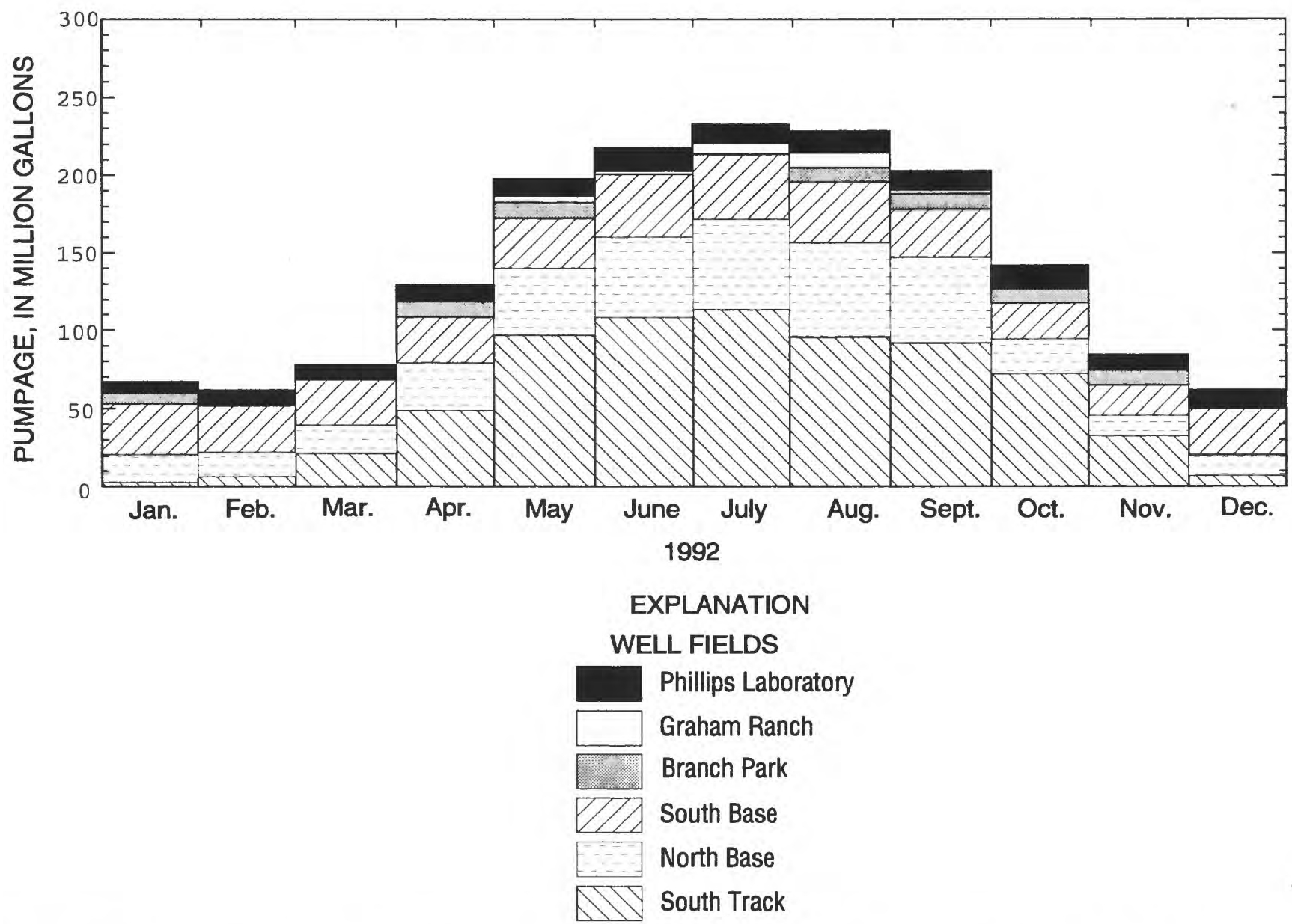

Figure 7. Total monthly pumpage at Edwards Air Force Base, California. (See table 3 for pumpage values.)

The production wells in the South Track, South Base, Branch Park, and Phillips Laboratory well fields yield water from the deep aquifer of the Lancaster subbasin, and the production wells in the North Base well field yield water from the unconfined aquifer of the North Muroc subbasin. Production wells in the Graham Ranch well field yield water from an unconfined aquifer in a small isolated subbasin. Total base pumpage for EAFB in 1992 was about 1,700 million gal, or 5,225 acre-ft (table 3) (Ronald Johnson, Edwards Air Force Base, written commun., 1993; C. Singletary, Superintendent of Water and Waste, Civil Engineering, Phillips Laboratory, Edwards Air Force Base, written commun., 1993). Figure 7 is a bar graph that illustrates the seasonal fluctuations of monthly pumpage totals for the well fields presented in table 3 .

\section{South Track Well Field}

Total pumpage for 1992 for the South Track well field was about 697.3 million gal, 2,140 acre-ft (table 3). Hydrographs for wells and selected piezometers in and near the South Track well field and total monthly pumpage for wells $8 \mathrm{~N} / 10 \mathrm{~W}-1 \mathrm{C} 2$ (S-6), 9N/ $10 \mathrm{~W}-36 \mathrm{~F} 1 \mathrm{(S}-4)$, and $-36 \mathrm{P} 1$ (S-5) are shown in figure 8. During winter and early spring recovery, pumpage was low and hydraulic heads were about 2,164 to $2,166 \mathrm{ft}$ above sea level. From April to May, pumpage doubled and heads began to decline (fig. 8). Pumping was nearly continuous from May into October. From April to September, heads declined about $9 \mathrm{ft}$ in piezometers $8 \mathrm{~N} / 10 \mathrm{~W}-1 \mathrm{Q} 3$ and $9 \mathrm{~N} / 10 \mathrm{~W}-25 \mathrm{P} 1$ and about $10 \mathrm{ft}$ in wells $9 \mathrm{~N} / 10 \mathrm{~W}-36 \mathrm{~F} 1$ and $8 \mathrm{~N} / 10 \mathrm{~W}-1 \mathrm{C} 2$. Draw- 
Table 3. Monthly and annual pumpage data from production wells on Edwards Air Force Base, California, 1992 [State well No.: See well-numbering system in text. See figure 5 for locations of wells. n/o, pump not operational; Mgal,

\begin{tabular}{|c|c|c|c|c|c|c|c|}
\hline \multirow{2}{*}{ State well No. } & \multirow{2}{*}{$\begin{array}{c}\text { Base well } \\
\text { identification } \\
\text { No. }\end{array}$} & \multicolumn{6}{|c|}{ Pumpage, in million gallons } \\
\hline & & January & February & March & April & May & June \\
\hline \multicolumn{8}{|c|}{ South Track well field } \\
\hline $8 \mathrm{~N} / 10 \mathrm{~W}-1 \mathrm{C} 2$ & S-6 & 1.017 & 2.343 & 3.671 & 8.716 & 41.609 & 6.925 \\
\hline $9 \mathrm{~N} / 10 \mathrm{~W}-36 \mathrm{~F} 1$ & S-4 & .415 & 3.988 & 5.064 & 30.782 & 10.589 & 42.034 \\
\hline$-36 \mathrm{P} 1$ & S-5 & 1.336 & 0 & 12.723 & 9.277 & 44.751 & 59.458 \\
\hline \multicolumn{2}{|r|}{ Total } & 2.768 & 6.331 & 21.458 & 48.775 & 96.949 & 108.417 \\
\hline \multicolumn{8}{|l|}{ North Base well field } \\
\hline \multirow[t]{2}{*}{$10 \mathrm{~N} / 9 \mathrm{~W}-5 \mathrm{~B} 1$} & \multirow[b]{2}{*}{ Total } & 17.573 & 15.469 & 17.596 & 30.111 & 42.917 & 51.553 \\
\hline & & 17.573 & 15.469 & 17.596 & 30.111 & 42.917 & 51.553 \\
\hline \multicolumn{2}{|l|}{ South Base well field } & & & & & & \\
\hline 9N/10W-24E2 & S-3 & 0 & 0 & 0 & 0.908 & 3.013 & 13.340 \\
\hline \multirow[t]{2}{*}{$-24 \mathrm{G} 1$} & \multirow[b]{2}{*}{ Total } & 32.627 & 29.952 & 29.464 & 28.629 & 29.394 & 27.366 \\
\hline & & 32.627 & 29.952 & 29.464 & 29.537 & 32.407 & 40.706 \\
\hline \multicolumn{8}{|c|}{ Branch Park well field } \\
\hline \multirow[t]{2}{*}{ 9N/10W-34P3 } & \multirow[b]{2}{*}{ Total } & 6.925 & 0 & 0 & 10.121 & 10.212 & 1.886 \\
\hline & & 6.925 & 0 & 0 & 10.121 & 10.212 & 1.886 \\
\hline \multicolumn{8}{|c|}{ Graham Ranch well field } \\
\hline \multirow{3}{*}{$\begin{array}{r}9 \mathrm{~N} / 10 \mathrm{~W}-16 \mathrm{P} 1 \\
-16 \mathrm{R} 4\end{array}$} & C-3 & 0.057 & 0.063 & 0.019 & 0.328 & 4.249 & 0.992 \\
\hline & C-4 & n/o & $\mathrm{n} / \mathrm{o}$ & n/o & $\mathrm{n} / \mathrm{o}$ & $\mathrm{n} / \mathrm{o}$ & $\mathrm{n} / \mathrm{o}$ \\
\hline & Total & 0.057 & 0.063 & 0.019 & 0.328 & 4.249 & 0.992 \\
\hline \multicolumn{8}{|c|}{ Phillips Laboratory well fields } \\
\hline $9 \mathrm{~N} / 9 \mathrm{~W}-14 \mathrm{P} 2$ & Well B & 2.442 & 1.123 & 2.758 & 2.628 & 2.457 & 4.748 \\
\hline$-15 \mathrm{~J} 1$ & Well A & 1.176 & 3.784 & 1.603 & 3.058 & 2.233 & 2.748 \\
\hline$-13 \mathrm{~N} 1$ & Well D & n/o & $\mathrm{n} / \mathrm{o}$ & n/o & n/o & .421 & .278 \\
\hline$-14 Q 1$ & Well C & 1.981 & 2.771 & 2.448 & 2.423 & 2.473 & 3.170 \\
\hline \multirow[t]{2}{*}{$9 \mathrm{~N} / 8 \mathrm{~W}-6 \mathrm{~J} 1$} & MW-3 & 1.516 & 2.180 & 2.196 & 2.535 & 3.378 & 3.145 \\
\hline & Total & 7.115 & 9.858 & 9.005 & 10.644 & 10.962 & 14.089 \\
\hline \multicolumn{2}{|l|}{$\begin{array}{l}\text { Monthly base total, } \\
\text { million gallons......... }\end{array}$} & 67.065 & 61.673 & 77.542 & 129.516 & 197.696 & 217.643 \\
\hline $\begin{array}{l}\text { Monthly base total, } \\
\text { acre-feet.................... }\end{array}$ & 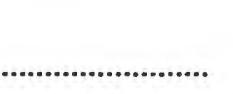 & 205.8 & 189.2 & 237.9 & 397.4 & 606.6 & 667.8 \\
\hline
\end{tabular}

${ }^{1}$ Flowmeter not operational, pumpage estimated using number of hours operated at 1,700 gallons per minute. 
million gallons; acre-ft, acre-foot]

\begin{tabular}{|c|c|c|c|c|c|c|c|}
\hline \multicolumn{6}{|c|}{ Pumpage, in million gallons--Continued } & \multirow{2}{*}{$\begin{array}{l}\text { Annual well and } \\
\text { well field totals } \\
\text { (Mgal) }\end{array}$} & \multirow{2}{*}{$\begin{array}{l}\text { Annual well } \\
\text { field total } \\
\text { (acre-ft) }\end{array}$} \\
\hline July & August & September & October & November & December & & \\
\hline 0 & 0.00 & 2.542 & 4.920 & 2.023 & 4.358 & 78.124 & \\
\hline 56.054 & 53.576 & 31.404 & 19.377 & 5.303 & 2.615 & 261.201 & \\
\hline 57.330 & $42.073^{1}$ & $57.907^{1}$ & 47.868 & $25.214^{1}$ & $\mathrm{n} / \mathrm{o}$ & 357.937 & \\
\hline 113.384 & 95.649 & 91.853 & 72.165 & 32.540 & 6.973 & 697.262 & $2,139.6$ \\
\hline 58.116 & 60.812 & 55.149 & 21.988 & 12.757 & 13.140 & 397.181 & \\
\hline 58.116 & 60.812 & 55.149 & 21.988 & 12.757 & 13.140 & 397.181 & $1,218.7$ \\
\hline 14.092 & 11.434 & 5.119 & 10.289 & 7.831 & 1.876 & 67.902 & \\
\hline 27.841 & 28.048 & 25.883 & 13.133 & 11.629 & 27.819 & 311.785 & \\
\hline 41.933 & 39.482 & 31.002 & 23.422 & 19.460 & 29.695 & 379.687 & $1,165.0$ \\
\hline 0.00 & $8.869^{1}$ & 10.060 & 9.359 & 9.394 & 0.741 & 67.567 & \\
\hline 0.00 & 8.869 & 10.060 & 9.359 & 9.394 & 0.741 & 67.567 & 207.3 \\
\hline $\mathrm{n} / \mathrm{o}$ & $\mathrm{n} / \mathrm{o}$ & 0 & 0.075 & 0 & 0 & 5.783 & \\
\hline 7.074 & 9.712 & 2.468 & 0 & .825 & .362 & 20.441 & \\
\hline 7.074 & 9.712 & 2.468 & 0.075 & 0.825 & 0.362 & 26.224 & 80.5 \\
\hline 3.018 & 2.784 & 2.035 & 2.210 & 1.346 & 1.494 & 29.043 & \\
\hline 1.136 & 3.121 & 2.371 & 3.770 & 1.984 & 2.590 & 29.574 & \\
\hline 2.797 & 3.719 & 3.295 & 3.725 & 2.162 & 2.881 & 33.529 & \\
\hline 4.432 & 2.984 & 3.500 & 2.801 & 1.940 & 2.762 & 19.118 & \\
\hline .758 & 1.314 & 1.241 & 2.406 & 1.751 & .925 & 23.661 & \\
\hline 12.141 & 13.922 & 12.442 & 14.912 & 9.183 & 10.652 & 134.925 & 414.0 \\
\hline 232.648 & 228.446 & 202.974 & 141.921 & 84.159 & 61.563 & $1,702.846$ & \\
\hline 713.9 & 701.0 & 622.8 & 435.5 & 258.2 & 188.9 & $5,255.1$ & \\
\hline
\end{tabular}




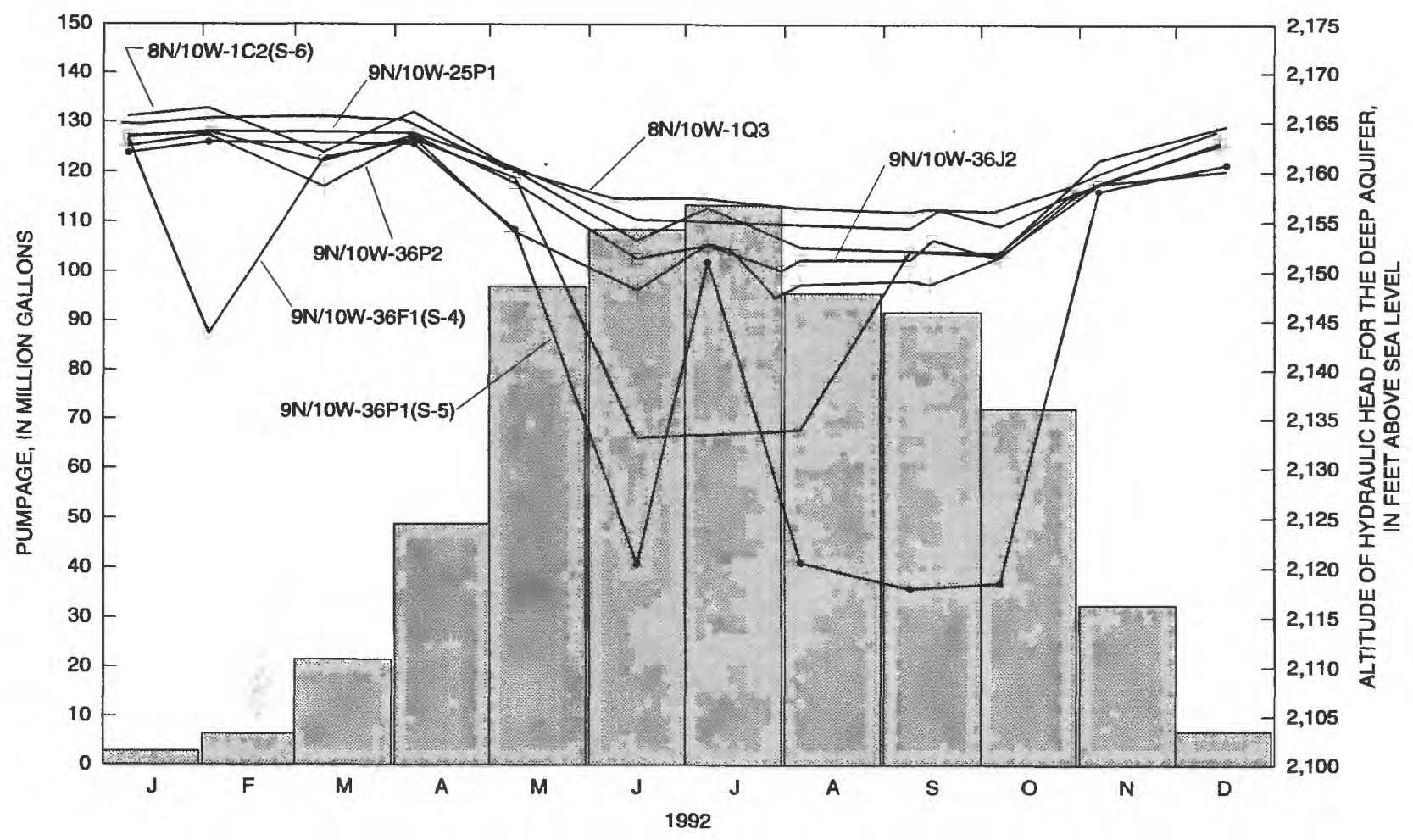

Figure 8. Monthly pumpage from and hydraulic heads in wells and selected piezometers in and near the South Track well field, Edwards Air Force Base, California.

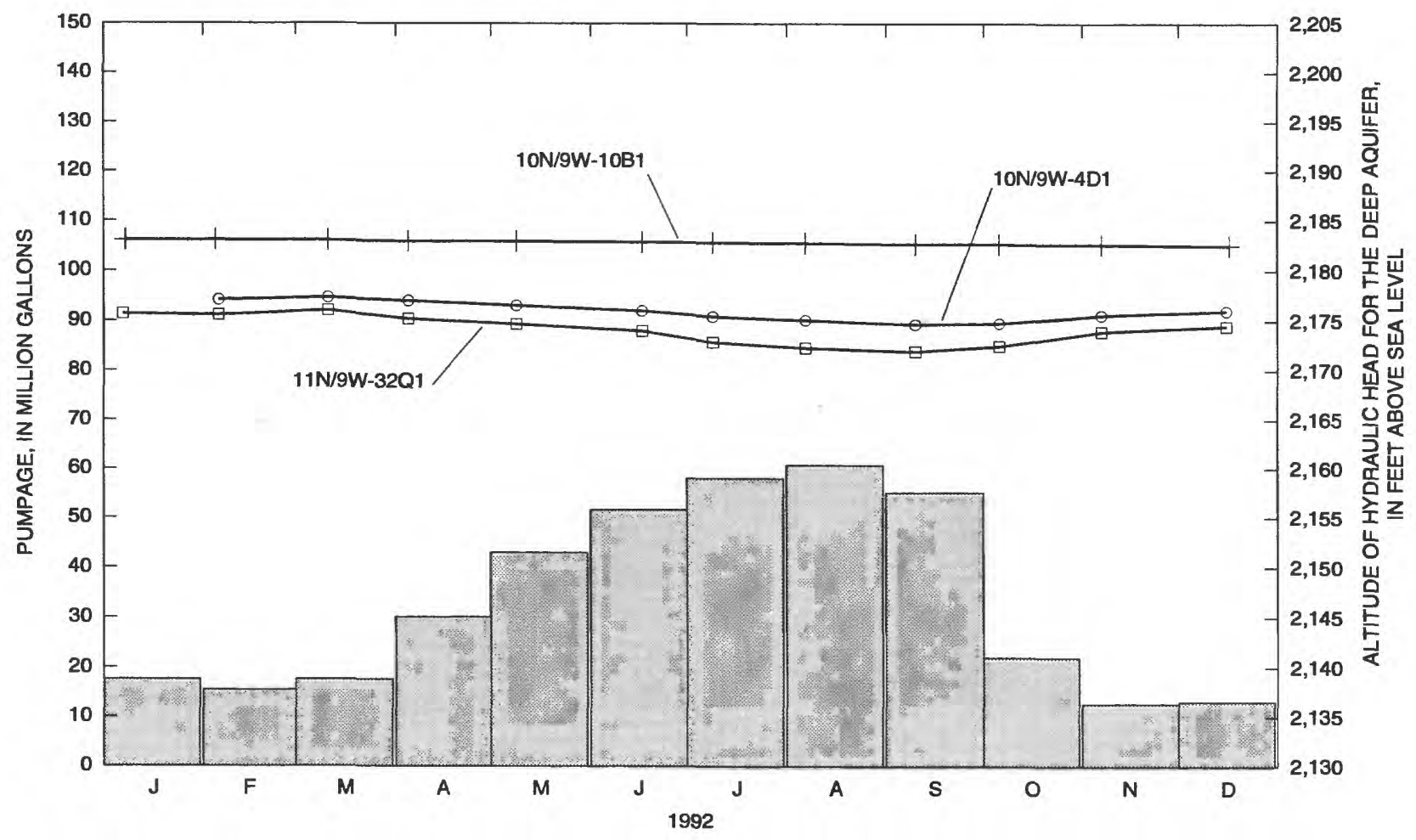

Figure 9. Monthly pumpage from and hydraulic heads in wells and selected piezometers in and near the North Base well field, Edwards Air Force Base, California. 


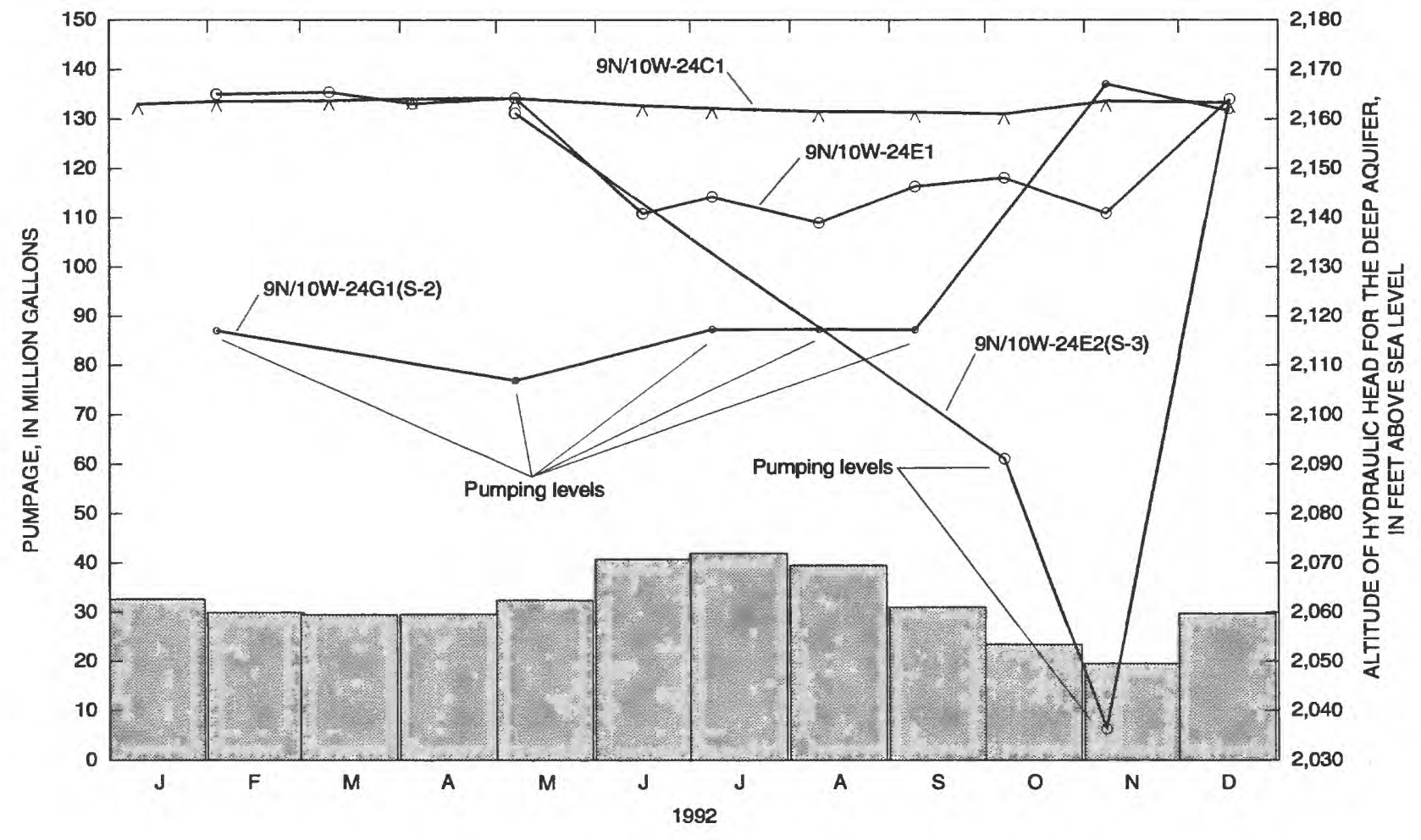

Figure 10. Monthly pumpage from and hydraulic heads in wells in the South Base well field, Edwards Air Force Base, California.

downs were about $20 \mathrm{ft}$ and $30 \mathrm{ft}$ in wells $9 \mathrm{~N} / 10 \mathrm{~W}$ $36 \mathrm{~F} 1$ and 9N/10W-36P1, respectively. Recovery began in late October, and by early December, heads recovered to about 2,160 to $2,165 \mathrm{ft}$ above sea level.

\section{North Base Well Field}

About 397.2 million gal, or 1,219 acre-ft, was pumped from well 10N/9W-5B1 in the North Base well field (fig. 5) in 1992 (table 3). From April to September 1992, hydraulic heads declined about $2 \mathrm{ft}$ in well $10 \mathrm{~N} / 9 \mathrm{~W}-4 \mathrm{D} 1$ to the east of well $10 \mathrm{~N} / 9 \mathrm{~W}-5 \mathrm{~B} 1$ and about $3 \mathrm{ft}$ in well $11 \mathrm{~N} / 9 \mathrm{~W}-32 \mathrm{Q} 1$ to the north of well 10N/9W-5B1 (fig. 9). By December, well 10N/ 9W-4D1 had recovered $1.3 \mathrm{ft}$ and well $11 \mathrm{~N} / 9 \mathrm{~W}-32 \mathrm{Q} 1$ had recovered $2.2 \mathrm{ft}$. Heads in piezometer $10 \mathrm{~N} / 9 \mathrm{~W}-$ $10 \mathrm{~B} 1$, about $2.2 \mathrm{mi}$ southeast of well $10 \mathrm{~N} / 9 \mathrm{~W}-5 \mathrm{~B} 1$, declined steadily about half a foot during an 11-month period (January 8 to December 17, 1992).

\section{South Base Well Field}

Total annual pumpage for the South Base well field (fig. 5) in 1992 was about 379.7 million gal,
1,165 acre-ft (table 3 ), with an average monthly total of about 31.6 million gal, 97 acre-ft. The hydrograph for well 9N/10W-24C1 (fig. 10) shows that hydraulic heads ranged from about 2,164 to $2,167 \mathrm{ft}$ above sea level. The altitude scale in figure 10 is two times that of figures 8 and 9 . Drawdowns ranged about 50 to 60 $\mathrm{ft}$ for well 9N/10W-24G1 (S-2) and about 70 to $130 \mathrm{ft}$ for 9N/10W-24E2 (S-3). Well 9N/10W-24E1 is about $750 \mathrm{ft}$ north of well 9N/10W-24E2 (fig. 5). The 10foot drop in head in well 9N/10W-24E1 when well $-24 \mathrm{E} 2$ (S-3) was being pumped indicates that -24E1 is in the cone of depression of -24E2. Large drawdowns in both production wells may be due to low transmissivities (Londquist and others, 1993, tables 7 and 8) in this area or may indicate loss of storage in the aquifer because of dewatering and compaction of the interbedded, fine-grained layers. Large drawdowns allow an increase in oxidation or corrosion of the steel well casing when it is exposed to air, which could lead to collapse or shearing of the well casing. Such corrosion may have contributed to the collapse of well $9 \mathrm{~N} / 9 \mathrm{~W}$ $18 \mathrm{C} 1$ (S-1) in 1991. The cement pump pad for well 9N/10W-24E2 was cracked and the cement foundation 


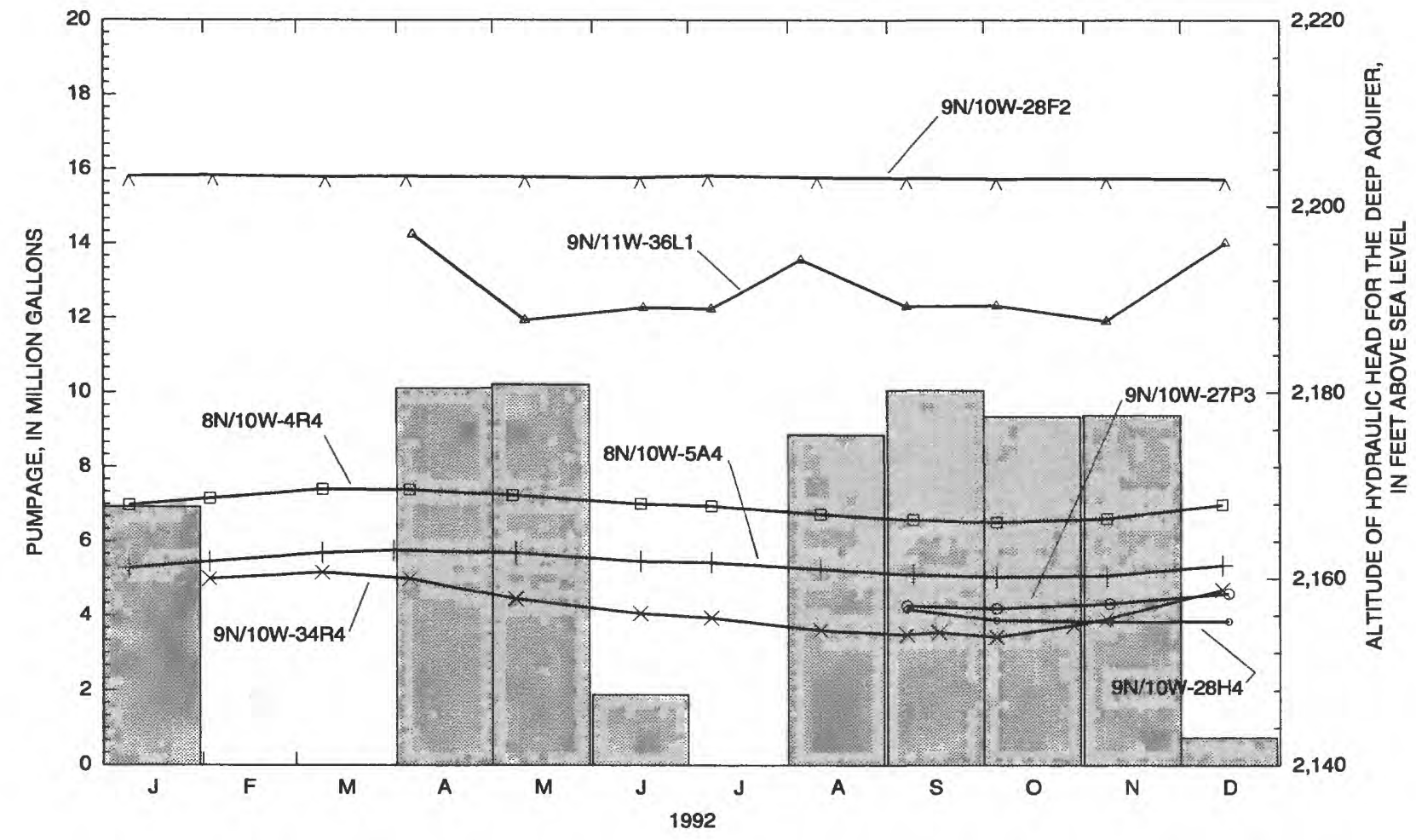

Figure 11. Monthly pumpage from and hydraulic heads in wells and selected piezometers in and near the Branch Park well field, Edwards Air Force Base, California.

had broken away from the bottom of the pad about 1 to 2 in., which indicates land subsidence and surface deformation have occurred around this well.

\section{Branch Park Well Field}

The Branch Park well field is about $1.5 \mathrm{mi}$ west of the South Track well field (fig. 5). About 67.6 million gal, or 207 acre- $\mathrm{ft}$, of ground water was pumped from well 9N/10W-34P3 (C-1) (table 3). The pumpage scale in figure 11 is one-fifth the pumpage scale in figures 8,9 , and 10 . Hydraulic head in piezometer $9 \mathrm{~N} /$ 10W-34R4 declined about $7 \mathrm{ft}$ from 2,160 to $2,153 \mathrm{ft}$ above sea level between March and October 1992, then started to recover in late October even though well 9N/10W-34P3 continued to be pumped through November. This fluctuation reflects the influences of the combined pumping stresses occurring in the South Track and Branch Park well fields. Head in well 9N/ $10 \mathrm{~W}-28 \mathrm{~F} 2$, about $2 \mathrm{mi}$ to the northwest of the Branch Park well field, was about $2,203 \mathrm{ft}$ above sea level and about 40 to $50 \mathrm{ft}$ higher than heads in piezometers $8 \mathrm{~N} /$ $10 \mathrm{~W}-5 \mathrm{~A} 4$ and 9N/10W-34R4. Head in well 9N/10W$28 \mathrm{~F} 2$ did not respond to seasonal pumping stresses (fig. 11). These observations could be explained if well 9N/10W-28F2 were isolated from the aquifer system of the Lancaster subbasin.

Well 9N/11W-36L1, about $4 \mathrm{mi}$ west of the Branch Park well field, is believed to be completed in the deep aquifer. The cause of the erratic groundwater-level fluctuations in this well is unknown (fig. 11). Falling water was heard when water levels were more than $100 \mathrm{ft}$ below land surface, but no sound was heard when water levels were less than $95 \mathrm{ft}$ below surface, which may indicate a perched aquifer in this area. This well may act as a conduit that hydraulically connects the perched and deep aquifers.

\section{Graham Ranch Well Field}

About 26.2 million gal, 80 acre-ft, were pumped from wells 9N/10W-16P1 (C-3) and -16R4 (C-4) in the Graham Ranch well field (fig. 5) in 1992 (table 3). Hydraulic heads ranged from about 2,200 to $2,210 \mathrm{ft}$ above sea level (fig. 12). The altitude and pumpage scales in figure 12 are the same scales as those used in figure 11. About 0.3 million gal in April and about 4.2 million gal in May 1992 was pumped from well 9N/ 


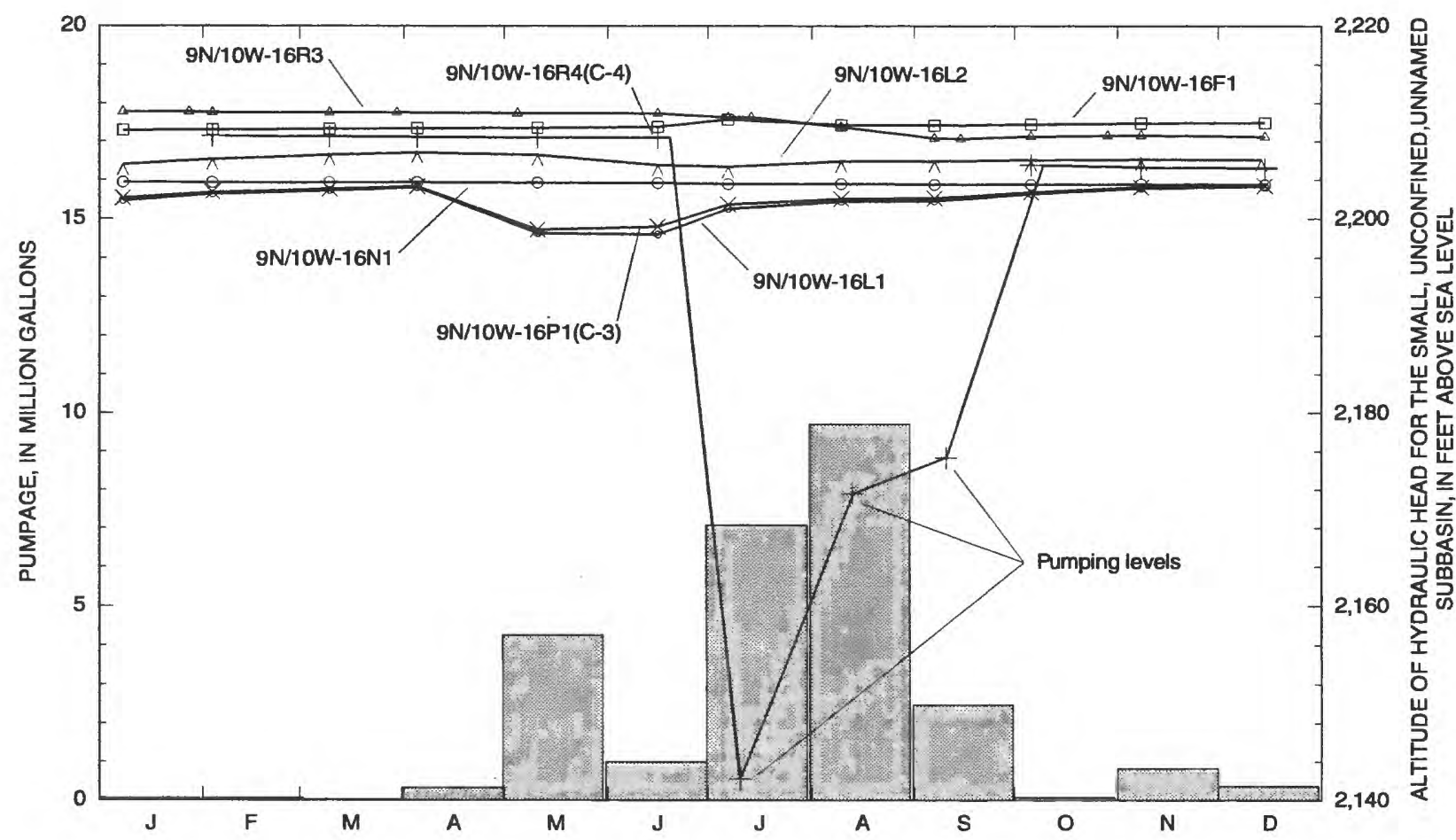

Figure 12. Monthly pumpage from and hydraulic heads in wells and selected piezometers in and near the Graham Ranch well field, Edwards Air Force Base, California.

$10 \mathrm{~W}-16 \mathrm{P} 1$ (C-3); the heads in wells 9N/10W$16 \mathrm{P} 1$ and $-16 \mathrm{~L} 1 \mathrm{declined}$ about $4.5 \mathrm{ft}$. Pumping from well 9N/10W-16P1 was ceased on June 30, 1992, and head was slow to recover to prepumping levels. Well 9N/10W-16R4 (C-4), 0.75 mi east of 9N/10W-16P1, was put into production July 1, 1992 (Ronald Johnson, Edwards Air Force Base, written commun., 1992). Drawdowns in well $9 \mathrm{~N} / 10 \mathrm{~W}-16 \mathrm{R} 4$ ranged from 30 to $65 \mathrm{ft}$, and recovery in October 1992 was about $3 \mathrm{ft}$ lower than prepumping levels.

\section{Phillips Laboratory Well Fields}

The Phillips Laboratory well fields are east of Rogers Lake (fig. 5). Two of the four production wells near the eastern shore of Rogers Lake were monitored, and one production well about $3.5 \mathrm{mi}$ to the northeast was monitored. These well fields produced about 134.9 million gal, or 414 acre-ft, in 1992 (table 3). The pumpage scale in figure 13 is the same scale as the scale used for figures 11 and 12. Hydraulic heads ranged from about 2,182 to $2,191 \mathrm{ft}$ above sea level (fig. 13). The altitude scale in figure 13 is three-fifths the scale used in figures $8,9,11$, and 12 .

Hydraulic heads in well 9N/8W-6J1 declined about $1 \mathrm{ft}$ from January to December 1992; drawdown was about $23 \mathrm{ft}$. Heads in the wells and piezometers in and near the Phillips Laboratory well fields declined about 1 to $2 \mathrm{ft}$ from January to December. Heads in piezometer 9N/9W-9A2 and production well 9N/9W15J1 (Well A) declined about $1 \mathrm{ft}$ between June and July (fig. 13) after production well 9N/9W-13N1 (Well D) began being pumped heavily at the end of June (table 3) (C. Singletary, Superintendent of Water and Waste, Civil Engineering, Phillips Laboratory, Edwards Air Force Base, written commun., 1993). This pumping had no influence on heads in wells 9N/ 9W-10R1 and -27H1 (fig. 13), about $1 \mathrm{mi}$ north and $1.5 \mathrm{mi}$ south of the well field, respectively. The heads in piezometers 9N/9W-9A1 (fig. $6 K$ ) and 9N/9W$28 \mathrm{~A} 1$ through $-28 \mathrm{~A} 4$ (fig. $6 J$ ) also did not respond to 


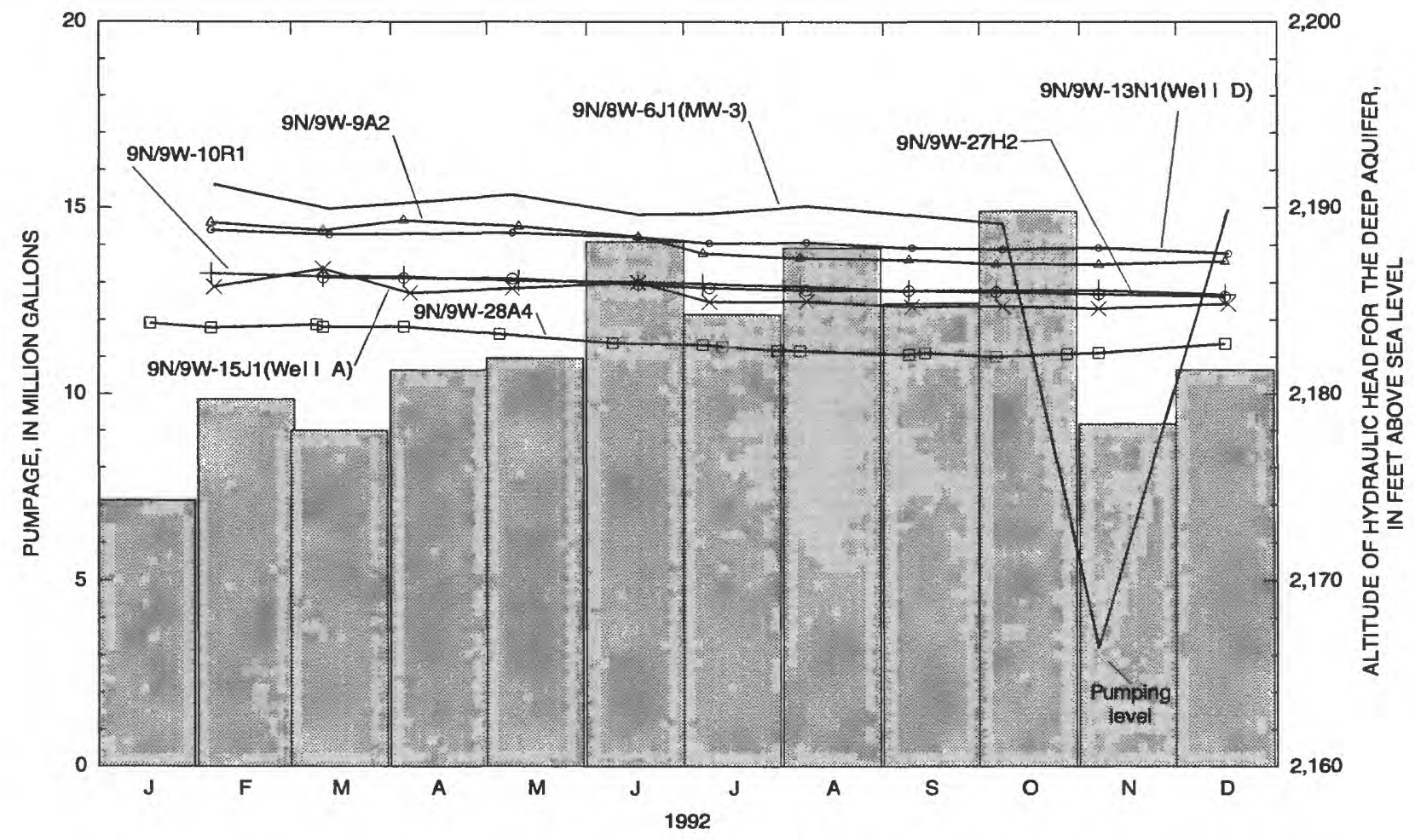

Figure 13. Monthly pumpage from and hydraulic heads in wells and selected piezometers in and near the Phillips Laboratory well fields, Edwards Air Force Base, California.

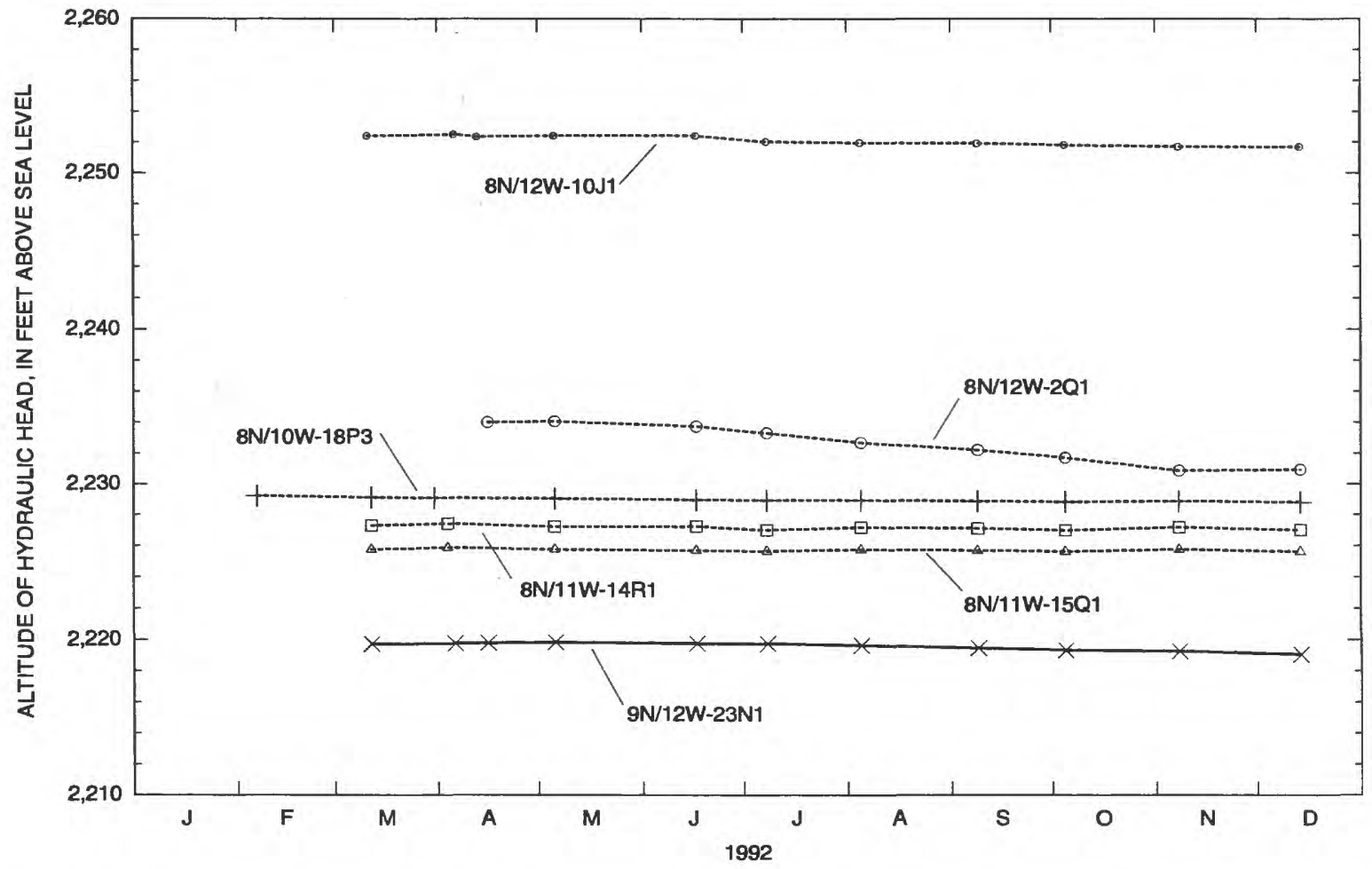

Figure 14. Hydraulic heads in wells south and west of Buckhorn and Rosamond Lakes, Edwards Air Force Base, California. 
the increase in pumping. Piezometer 9N/9W-9A2 and well 9N/9W-15J1 seem to be hydraulically connected to well 9N/9W-13N1, and wells 9N/9W-10R1 and $27 \mathrm{H} 2$ and piezometers $9 \mathrm{~N} / 9 \mathrm{~W}-9 \mathrm{~A} 1$ and $-28 \mathrm{~A} 1$ do not seem to be connected. This hydraulic connection between the production wells and piezometer 9N/9W9A2 may be through channelized, unconsolidated, coarse-grained gravel with high transmissivity, while wells 9N/9W-10R1 and -27H1 may be completed in poorly to moderately consolidated, fine-grained sediments that have lower transmissivities. Further study is needed to understand the variability in transmissivities in this area.

\section{Hydraulic Heads in Wells Southeast and West of Rosamond Lake}

Wells 8N/10-18P3, 8N/11W-14R1, -15Q1, 8N/ $12 \mathrm{~N}-2 \mathrm{Q} 1$, and $-10 \mathrm{~J} 1$ (fig. 5) are completed in the principal aquifer (table 1). Hydraulic heads in wells $8 \mathrm{~N}$ / 10W-18P3, 8N/11W-14R1 and -15Q1, and 8N/12W$10 \mathrm{~J} 1$ (fig. 14) remained relatively static from February and March 1992 through December 1992. Head in well $8 \mathrm{~N} / 12 \mathrm{~W}-2 \mathrm{Q} 1$ declined about $3 \mathrm{ft}$ from April to November (table 2).

Well 9N/12W-23N1 is near the northwest shore of Rosamond Lake (fig. 5). The location and depth to water of this well suggest that it is completed in the deep aquifer (tables 1 and 2). Heads in this well declined about $0.66 \mathrm{ft}$ between March and December 1992 (table 2, fig. 14). Figure 14 shows heads in well 9N/12W-23N1 relative to those in wells $8 \mathrm{~N} / 12 \mathrm{~W}-2 \mathrm{Q} 1$ and $-10 \mathrm{~J} 1,3$ and $3.5 \mathrm{mi}$ south, respectively, which are completed in the principal aquifer. Historical records indicate that heads in well 9N/12W-23N1 were similar to heads in wells $8 \mathrm{~N} / 12 \mathrm{~W}-2 \mathrm{Q} 1$ and $-10 \mathrm{~J} 1$ in the late 1950 's and early 1960 's. The rate of head decline in wells $8 \mathrm{~N} / 12 \mathrm{~W}-2 \mathrm{Q} 1$ and $-10 \mathrm{~J} 1$ slowed in the early 1970 's and then leveled off in the early 1980 's (Londquist and others, 1993, fig. 17). This leveling off corresponds to a decline in agricultural activities in the valley. Heads in well 9N/12W-23N1 continued to decline in the deep aquifer. In 1992, heads in well 9N/ $12 \mathrm{~W}-23 \mathrm{~N} 1$ were about 13 and $33 \mathrm{ft}$ lower than those in wells 8N/12-2Q1 and -10J1, respectively (fig. 14). Lack of sufficient recharge, recharge capture, and increased pumping from the deep aquifer for public and industrial supply may explain the steady decline in heads in the deep aquifer. Continued monitoring and analysis of head levels in these wells may help determine the cause of the declining heads, as well as document short- and long-term changes in the aquifer system.

\section{BASIN BOUNDARIES}

Three types of no-flow boundaries have been identified for the aquifer system at EAFB: structural boundaries, a principal-aquifer boundary, and groundwater divides (fig. 3). A no-flow boundary is a specialized constant-flux boundary where flux is zero and is typified by a region across which ground water neither enters nor leaves the aquifer system. Structural boundaries are juxtaposed bedrock-alluvium or consolidated-unconsolidated alluvium. In the Lancaster subbasin near EAFB, flow in the deep aquifer is defined by structural boundaries and a ground-water divide. The principal-aquifer boundary is the contact between relatively thick, very fine-grained, low-permeability, lacustrine material of the confining unit and coarse-grained alluvium of the principal aquifer. The principal-aquifer boundary controls ground-water flow in the principal aquifer. The confining unit separates the principal and the deep aquifers of the Lancaster subbasin. Permeability contrasts across structural and principal-aquifer boundaries generally are greater than several orders of magnitude. A ground-water divide is a ridge of relatively high hydraulic heads in the aquifer along which hydraulic heads are equal and from which ground water flows in opposite directions. The ground-water divide controls ground-water flow in the deep aquifer between the Lancaster and North Muroc subbasins. Boundary conditions were determined using surface and borehole geophysical data, lithologic logs, and ground-water-level data. Table 4 lists the altitudes of the confining-unit interval and bedrock-alluvium contacts of the wells used in this study.

\section{Structural Boundaries}

The structural boundaries to the south and southeast of Rogers Lake and north of the Phillips Laboratory well fields (fig. 5) are bedrock-alluvium contacts. These boundaries were defined using isostatic residual gravity data (John Mariano, U.S. Geological Survey, written commun., 1991). Lithologic logs for wells $10 \mathrm{~N} / 8 \mathrm{~W}-32 \mathrm{R} 1$ and 9N/8W-6J1 show 
Table 4. Altitudes of confining-unit interval and bedrock-alluvium contacts for wells on and near Edwards Air Force Base, California

[State well No.: See well-numbering diagram on page V. See figure 5 for location of wells. Altitude of confining-unit interval and bedrock-alluvium contact in feet above sea level. (U.S. Army Corp of Engineers, 1961; Dutcher and others, 1962;

Dutcher and Worts, 1963; Londquist and others, 1993; Rewis, 1993]

\begin{tabular}{|c|c|c|c|c|c|}
\hline State well No. & $\begin{array}{l}\text { Altitude of } \\
\text { confining-unit } \\
\text { interval }\end{array}$ & $\begin{array}{c}\text { Altitude of } \\
\text { bedrock-alluvium } \\
\text { contact }\end{array}$ & State well No. & $\begin{array}{l}\text { Altitude of } \\
\text { confining-unit } \\
\text { interval }\end{array}$ & $\begin{array}{c}\text { Altitude of } \\
\text { bedrock-alluvium } \\
\text { contact }\end{array}$ \\
\hline $8 \mathrm{~N} / 9 \mathrm{~W}-6 \mathrm{D} 1$ & $2,137-2,247$ & & $9 \mathrm{~N} / 10 \mathrm{~W}-12 \mathrm{R} 1^{1}$ & & 2,032 \\
\hline $8 \mathrm{~N} / 10 \mathrm{~W}-1 \mathrm{Q} 1$ & $2,052-2,212$ & & $-14 C 1^{1}$ & $2,218-2,204$ & 2,204 \\
\hline$-1 \mathrm{Q} 2$ & $2,052-2,212$ & & $-16 \mathrm{~F} 1$ & & 2,190 \\
\hline$-1 \mathrm{Q} 3$ & $2,052-2,212$ & & $-16 L 3$ & & 2,073 \\
\hline$-1 Q 4$ & $2,052-2,212$ & & $-16 \mathrm{~N} 1$ & & 1,936 \\
\hline$-4 R 1$ & $2,071-2,261$ & & $-24 \mathrm{C} 1$ & $2,193-2,238$ & \\
\hline$-4 R 2$ & $2,071-2,261$ & & $-24 \mathrm{E} 1$ & $1,788-1,822$ & \\
\hline$-4 R 3$ & $2,071-2,261$ & & $-25 \mathrm{P} 1$ & $2,169-2,269$ & \\
\hline$-4 \mathrm{R} 4$ & $2,071-2,261$ & & $-27 \mathrm{P} 1$ & $2,239-2,278$ & \\
\hline$-8 \mathrm{~J} 1^{1}$ & $1,991-2,100$ & & $-27 \mathrm{P} 2$ & $2,239-2,278$ & \\
\hline$-17 \mathrm{~J} 2^{1}$ & $\left(^{2}\right)-2,129$ & & $-28 \mathrm{H} 3$ & $2,277-2,288$ & 2,025 \\
\hline$-18 \mathrm{~N} 1^{1}$ & (') $-2,059$ & & $-28 \mathrm{H} 4$ & $2,277-2,288$ & 2,025 \\
\hline$-19 \mathrm{~N} 2^{1}$ & $1,927-2,072$ & & $-34 \mathrm{P}^{1}$ & $2,150-2,295$ & \\
\hline$-28 \mathrm{~A} 1^{1}$ & (2) $-2,083$ & & $-34 R 2$ & $2,145-2,190$ & \\
\hline \multirow[t]{2}{*}{$-30 R 1^{1}$} & $1,706-2,104$ & & $-34 R 3$ & $2,145-2,190$ & \\
\hline & & & $-34 R 4$ & $2,145-2,190$ & \\
\hline $8 \mathrm{~N} / 11 \mathrm{~W}-9 \mathrm{D} 1^{1}$ & $1,976-2,176$ & & $-36 \mathrm{~J} 1$ & $2,143-2,233$ & \\
\hline$-10 \mathrm{E} 1^{1}$ & $1,937-2,274$ & & $-36 \mathrm{~J} 2$ & $2,143-2,233$ & \\
\hline \multirow[t]{2}{*}{$-22 \mathrm{P} 2^{1}$} & $\left({ }^{2}\right)-2,115$ & & $-36 \mathrm{~J} 3$ & $2,143-2,233$ & \\
\hline & & & $-36 \mathrm{P} 2$ & $2,135-2,270$ & \\
\hline $8 \mathrm{~N} / 12 \mathrm{~W}-13 \mathrm{D} 1^{1}$ & $1,887-2,283$ & & & & \\
\hline$-14 R 1^{1}$ & $1,911-2,291$ & & $9 \mathrm{~N} / 12 \mathrm{~W}-26 \mathrm{Q} 1^{1}$ & $2,171-2,284$ & \\
\hline$-24 \mathrm{P} 1^{1}$ & $1,646-2,234$ & & $-28 \mathrm{~F} 3^{1}$ & $2,254-2,324$ & \\
\hline 9N/8W-6J1 & & 2,024 & $10 \mathrm{~N} / 8 \mathrm{~W}-32 \mathrm{R} 1^{1}$ & & 2,336 \\
\hline $9 \mathrm{~N} / 9 \mathrm{~W}-6 \mathrm{C} 1^{1}$ & & 2.179 & $10 \mathrm{~N} / 9 \mathrm{~W}-4 \mathrm{D} 1$ & $\left(^{2}\right)-1,867$ & \\
\hline$-6 \mathrm{E} 1^{1}$ & & 2,208 & $-27 \mathrm{C} 1$ & $2,242-2,272$ & 2,082 \\
\hline$-6 \mathrm{~L} 1^{1}$ & & 2,151 & $-27 C 2$ & $2,242-2,272$ & 2,082 \\
\hline$-9 A 1$ & $2,196-2,271$ & & $-27 C 3$ & $2,242-2,272$ & 2,082 \\
\hline$-9 A 2$ & $2,196-2,271$ & & $-31 C 1^{1}$ & & 2,118 \\
\hline$-28 \mathrm{~A} 1$ & $2,186-2,271$ & & $-31 C 4^{1}$ & & 2,155 \\
\hline$-28 \mathrm{~A} 2$ & $2,186-2,271$ & & $-31 N 1^{1}$ & & 2,212 \\
\hline$-28 A 3$ & $2,186-2,271$ & & & & \\
\hline$-28 \mathrm{~A} 4$ & $2,186-2,271$ & & & & \\
\hline
\end{tabular}

${ }^{1}$ Wells not monitored for this study.

${ }^{2}$ Altitude of lower contact unknown. 
bedrock altitudes of 2,336 and 2,024 ft above sea level, respectively (table 4). The difference in landsurface altitude between these two wells is about $56 \mathrm{ft}$ (table 1); the difference in bedrock altitude is about $312 \mathrm{ft}$. This difference indicates a structural boundary, probably a fault, between these wells.

Isostatic residual gravity data also were used to define the structural boundary south of the Rosamond and Bissell Hills (fig. 3) (John Mariano, U.S. Geological Survey, written commun., 1991). This boundary strikes southwest-northeast from the eastern shore of Rosamond Lake to Buckhorn Lake and coincides with the northwestern boundary of the Antelope Valley Fault Zone defined by Gary Dixon (U.S. Geological Survey, written commun., 1993) (fig. 3). This boundary juxtaposes younger, more permeable alluvium on the south against older, less permeable alluvium on the north. Dibblee (1960) describes this older alluvium as a fanglomerate of early Pleistocene age.

The structural boundary extends across Buckhorn Lake and along the southeastern edge of Hospital Ridge and juxtaposes the granitic bedrock of Hospital Ridge against younger alluvium of the basin (Dibblee, 1960). Monthly heads in well 9N/10W-28F2 (fig. 11) were similar to heads in wells 9N/10W-16N1 and -16M1 (fig. 12) to the north, near the Graham Ranch well field, but were about 45 to $50 \mathrm{ft}$ higher than those in piezometers 8N/10W-5A4, 9N/10W-27P3, -28H4 and $-34 \mathrm{R} 4$ to the south and east (fig. 11), indicating well 9N/10W-28F2 is north of the structural boundary.

From Hospital Ridge, the structural boundary strikes northward (fig. 3), crosses the buried Bissell Hills-El Mirage Fault (Gary Dixon, U.S. Geological Survey, written commun., 1993) and then parallels exposed bedrock west of Rogers Lake. Lithologic logs indicate that the altitudes of the bedrock-alluvium contact in wells 9N/9W-6E1 and 10N/9W-31N1 are about 2,200 and $2,212 \mathrm{ft}$ above sea level, respectively (table 4). The altitudes of the bedrock-alluvium contact for wells 9N/9W-6C1 and -6L1, 9N/10W-12R1, and 10N/9W-31C1 are about 2,179, 2,151, 2,032, and $2,118 \mathrm{ft}$ above sea level, respectively. Wells $9 \mathrm{~N} / 9 \mathrm{~W}-$ 6A1 and 10N/9W-31C4 did not penetrate bedrock. The structural boundary is interpreted to be between wells 10N/9W-31N1 and 9N/9W-6C1, between wells 9N/9W-6E1 and -6L1, west of well 10N/9W-31C1, and northwest of well 9N/10W-12R1. The position of the structural boundary west and northwest of the
North Base well field is unknown because of a lack of data.

\section{Principal-Aquifer Boundary}

The principal aquifer is defined primarily by the principal-aquifer boundary. This boundary is the contact between the principal aquifer and the underlying fine-grained confining unit (fig. 4) and part of a structural boundary. The confining unit is assumed to be relatively impermeable both laterally and vertically in relation to the aquifers. Ground-water-level data (table 2) and lithologic data (table 4) were used to determine the position of the principal-aquifer boundary (fig. 3). Wells $8 \mathrm{~N} / 10-8 \mathrm{~J} 1$ and $-18 \mathrm{P} 3 ; 8 \mathrm{~N} / 11 \mathrm{~W}-14 \mathrm{R} 1$ and $-15 \mathrm{Q} 1 ; 8 \mathrm{~N} / 12 \mathrm{~W}-2 \mathrm{Q} 1,-10 \mathrm{~J} 1,-24 \mathrm{P} 1,-26 \mathrm{~F} 1$, and $-28 \mathrm{D} 1$; and 9N/12W-33P1, south and southwest of Rosamond and Buckhorn Lakes, were completed in the principal aquifer above or several feet into the confining unit and south of the principal-aquifer boundary. The deep aquifer is confined in this region. The confining unit is at or near land surface in wells or piezometers 8N/10W-4R1, 8N/11W-10E1, 8N/12W13D1 and -14R1, and 9N/12W-26Q1 and -28F3. The altitude of the confining-unit interval in well 8N/10W$8 \mathrm{~J} 1$ south of the principal-aquifer boundary was 1,991 to $2,100 \mathrm{ft}$ above sea level; in piezometer $8 \mathrm{~N} / 10 \mathrm{~W}-$ $4 \mathrm{R} 1$ north of the principal-aquifer boundary, the altitude of the confining-unit interval was 2,071 to 2,261 $\mathrm{ft}$ above sea level (table 4 ). The bottom contact of the confining unit comes to the surface near the south-central part of Rogers Lake (fig. 3). North of the South Track well field, the deep aquifer of the Lancaster subbasin is considered unconfined.

\section{Ground-Water Divide}

A ground-water divide, oriented east-west across the north-central part of Rogers Lake (fig. 3), separates the Lancaster and North Muroc subbasins and prevents ground water from flowing northward into, or southward out of, the North Muroc subbasin. The location of this divide may not be static, but probably migrates north and south over time in response to seasonal and long-term changes in ground-water levels in the Lancaster and North Muroc subbasins. Heads in piezometers 10N/9W-27C1 and -27C2 were about 2,193 ft above sea level, about 5 to $9 \mathrm{ft}$ higher than heads in piezometers 9N/9W-9A1 and -9A2 to the south and about 10 to $11 \mathrm{ft}$ higher than heads in 
piezometers $10 \mathrm{~N} / 9 \mathrm{~W}-10 \mathrm{~B} 1$ and $-10 \mathrm{~B} 2$ to the north (fig. 6K-6M).

The ground-water divide corresponds to a bedrock ridge of unknown extent and depth buried under relatively thin alluvium and playa sediments (L.C. Dutcher, U.S. Geological Survey, written commun., 1959; Bloyd, 1967). Quartz monzonite is exposed at the playa surface of Rogers Lake in the southeast corner of T. 10 N., R. 9 W. (sec. 20) (Dibblee, 1960, pl. 8). The borehole for piezometers $10 \mathrm{~N} / 9 \mathrm{~W}-27 \mathrm{C} 1$ through $-27 \mathrm{C} 3$, about $1.5 \mathrm{mi}$ east-southeast of the quartz monzonite outcrop, penetrated granitic bedrock at $190 \mathrm{ft}$ below the playa surface (Rewis, 1993). Drill cuttings from depths greater than $190 \mathrm{ft}$ below land surface were very fine- to very coarse-grained, very angular fragments of feldspar and quartz (Rewis, 1993). Interpretation of refraction data collected during a seismic survey near piezometers $10 \mathrm{~N} / 9 \mathrm{~W}-27 \mathrm{C} 1$ through -27C3 indicated a near horizontal alluviumbedrock contact about $200 \mathrm{ft}$ below land surface (David Berger, U.S. Geological Survey, written commun., 1992). This alluvium-bedrock contact, a slow drilling rate, and borehole resistivity values greater than 150 ohm-meters (Rewis, 1993) corroborate the existence of a buried ridge and help to determine the lateral extent and depth of the ridge.

Contrary to the evidence mentioned above, an interpretation of data collected during a direct current resistivity survey on Rogers Lake near the groundwater divide indicates that the depth to high-resistivity bedrock materials is about 2,300 to $2,900 \mathrm{ft}$ below land surface (Zhody and Bisdorf, 1991). This resistivity data suggests that the bedrock outcrop and the material encountered during drilling may be large granitic boulders in the alluvium, but does not explain the distinct and extensive seismic-velocity contrast at 200 $\mathrm{ft}$ below land surface. This contrast may result because the material below $200 \mathrm{ft}$ is fractured, highly weathered, altered or saturated bedrock. The exposed bedrock in the surrounding area is extensively faulted and highly weathered, and there are volcanic and hydrothermal alterations of sediments and bedrock to the north of Rogers Lake, which could account for the lower resistivites of the material at depths.

The Graham Ranch well field (fig. 5) is south of the Bissell Hills and northwest of Hospital Ridge. Bloyd (1967) and Duell (1986) considered this area to be part of the deep aquifer in the Lancaster subbasin.
The Graham Ranch well field is separated topographically from Rogers Lake by the exposed bedrock of Hospital Ridge. Land-surface altitudes in this well field are about 40 to $50 \mathrm{ft}$ higher than the playa surface of Rogers Lake. The aquifer in the Graham Ranch well field is unconfined and probably is isolated from the deep and principal aquifers. Hydraulic heads are higher than those in the deep aquifer and lower than those in the principal aquifer. Heads range from about 2,200 to $2,215 \mathrm{ft}$ above sea level (fig. 12). The boundaries of this basin are irregularly shaped bedrock-alluvium contacts. The altitudes of the bedrock-alluvium contact in wells $9 \mathrm{~N} / 10 \mathrm{~W}-16 \mathrm{~F} 1,-16 \mathrm{~L} 3$, and $-16 \mathrm{~N} 1$ are $2,190,2,073$, and $1,936 \mathrm{ft}$ above sea level, respectively (table 4). Wells 9N/10W-16P1, -16L2, -16R1, and -16R4 were drilled deeper (table 1) (Dutcher and others, 1962; Londquist and others, 1993), not penetrating bedrock. The absence of bedrock in these four wells indicates the presence of a small, possibly narrow, down-dropped basin, or graben, with a minimum of $460 \mathrm{ft}$ of normal slip. This graben may be the result of the release of extensional stresses related to the Antelope Valley Fault Zone (fig. 2).

The Antelope Valley Fault Zone is a zone of left lateral strike slip faulting, with oblique dip-slip down to the southwest (Gary Dixon, U.S. Geological Survey, written commun., 1993). For the depth and configuration of this basin, the reader is referred to Londquist and others (1993). Gravity and surfaceresistivity data (Zhody and Bisdorf, 1990; J. Mariano, R.C., Joahens, and R.L. Morin, U.S. Geological Survey, written commun., 1991) indicate that there may be a hydraulic connection to the deep aquifer between the bedrock ridges, possibly through buried drainage channels, although this has yet to be verified (Londquist and others, 1993).

Water levels were used to identify a small northsouth trending ground-water divide in the Graham Ranch well field near piezometers 9N/10W-16R1 through -16R3. The borehole for the piezometers was drilled to $960 \mathrm{ft}$ below land surface and did not penetrate bedrock (Londquist and others, 1993). This ground-water divide is caused by two pumping centers around EAFB production wells $9 \mathrm{~N} / 10 \mathrm{~W}-16 \mathrm{P} 1(\mathrm{C}-3)$ and 9N/10W-16R4 (C-4). 


\section{SEASONAL POTENTIOMETRIC SURFACES}

A potentiometric surface is defined as an imaginary surface represented by hydraulic heads in wells and piezometers completed in an aquifer. For a confined aquifer, the potentiometric surface is above the base of a confining unit and is represented by the level to which water would rise in an open well penetrating the confined aquifer. For an unconfined aquifer, the potentiometric surface is the water table where ground-water pressures generally are the same as atmospheric pressure. For the purposes of this study, the heads of perched aquifers were not used to define the potentiometric surfaces because the water is trapped above the true water table by a lens of material with low permeability.

The purpose of mapping potentiometric surfaces is to provide a visual interpretation of the areal extent and generalized ground-water-flow paths of the aquifer system. Hydraulic heads in EAFB production wells, abandoned wells, and one piezometer from each of the USGS piezometer sites were used to contour the potentiometric surfaces (figs. 15 through 18, at back of report). Table 5 lists the hydraulic heads and changes in head used in this interpretation. The head values were rounded to the nearest tenth of a foot.

Several factors were used in the determination of the potentiometric surfaces of the aquifer system at EAFB. Pumping centers on the base were identified. Domestic and public supply wells in and near the town of Rosamond (fig. 2) and agricultural irrigation wells south of Redman also were considered because of their influence on the ground-water-flow paths and changes to the potentiometric surfaces. To project contours south and west of the base boundary (figs. 15 through 18), hydraulic heads were calculated for the principal and deep aquifers using spring groundwater-level measurements (tables 2 and 5) for wells monitored by the USGS as part of the Antelope Valley-East Kern Water Agency ground-water-monitoring program.

\section{Changes in the Potentiometric Surfaces}

Changes in the potentiometric surfaces of the aquifer system at EAFB were relatively small in 1992. Hydraulic head contours for spring 1992 ranged from about 2,160 to $2,220 \mathrm{ft}$ above sea level in the deep aquifer and 2,200 to 2,280 $\mathrm{ft}$ above sea level in the principal aquifer in the Lancaster subbasin; 2,180 to $2,190 \mathrm{ft}$ above sea level in the North Muroc subbasin; and 2,210 to $2,290 \mathrm{ft}$ above sea level in the Graham Ranch well-field area (figs. 3 and 15-18). Figure 19 shows contours for changes in hydraulic head for spring to late summer 1992 for wells completed in the deep aquifer (table 5). Changes in head for five wells completed in the principal aquifer (not illustrated) were less than $2 \mathrm{ft}$ (table 5). Figure 20 is generalized geologic cross sections showing hydraulic heads in selected wells and piezometers for sections shown on figure 15 .

\section{Deep Aquifer}

In the areas between and adjacent to the South Track, South Base, and Branch Park well fields, the potentiometric surface of the deep aquifer for spring 1992 ranged from 2,160 to $2,180 \mathrm{ft}$ above sea level forming a shallow regional ground-water depression (fig. 15). This depression is oriented along a southwest-northeast trending axis similar to the trend of maximum measured subsidence reported by Londquist and others (1993, fig. 21).

In spring 1992, the deep aquifer was confined in the South Track well field. A small, shallow, local ground-water depression formed around well 9N/ $10 \mathrm{~W}-34 \mathrm{P} 3$ in the Branch Park well field in response to pumping (table 3, figs. 15 and 20). In May, the South Track well field began pumping almost continuously. Between April and September, the potentiometric surface had declined about $10 \mathrm{ft}$ in the South Track well field, about $3 \mathrm{ft}$ in the South Base well field, and about $8 \mathrm{ft}$ in the Branch Park well field (table 5, fig. 19). Because of a 10-foot decline in the potentiometric surface in the South Track well field by late summer and 20 - to 30-foot drawdowns in production wells $8 \mathrm{~N} /$ $10 \mathrm{~W}-1 \mathrm{C} 2$ and $9 \mathrm{~N} / 10 \mathrm{~W}-36 \mathrm{~F} 1$ and -36P1 (table 5, fig. 8 ), water-levels dropped below the bottom of the confining unit, which resulted in the deep aquifer becoming locally unconfined near the wells (figs. 8 and 20).

The configuration of the potentiometric surface of the deep aquifer south and southwest of EAFB is unknown (figs. 15 and 16).

Change in hydraulic heads from spring to late summer in and near the Phillips Laboratory and North Base well fields were 0 to $2 \mathrm{ft}$ and 2 to $3 \mathrm{ft}$, respectively (fig. 19). Heads near the ground-water divide 
Table 5. Hydraulic heads and change in hydraulic heads for selected wells and piezometers used to plot the potentiometric surfaces of the aquifer system at Edwards Air Force Base, California, 1992

[State well No.: See well-numbering system on page V. See figures 15 through 18 for locations of wells. Hydraulic head, in feet, computed from land-surface altitude and depth to water (table 2), rounded to nearest tenth of a foot. Div., at the groundwater divide; ft, foot; do., ditto; --, data not available]

\begin{tabular}{|c|c|c|c|c|c|c|}
\hline \multirow{2}{*}{ State well No. } & \multirow{2}{*}{ Subbasin } & \multicolumn{2}{|c|}{ Hydraulic head, spring } & \multicolumn{2}{|c|}{ Hydraulic head, late summer } & \multirow{2}{*}{$\begin{array}{c}\text { Change in } \\
\text { hydraulic head } \\
\text { (ft) }\end{array}$} \\
\hline & & Date & Head & Date & Head & \\
\hline \multicolumn{7}{|c|}{ Completed in deep aquifer } \\
\hline $8 \mathrm{~N} / 10 \mathrm{~W}-1 \mathrm{C} 2$ & Lancaster & $4-07-92$ & $2,163.0$ & $9-09-92$ & $2,149.1$ & 13.9 \\
\hline$-1 \mathrm{Q} 3$ & do. & 4-04-92 & $2,165.2$ & $9-09-92$ & $2,156.0$ & 9.2 \\
\hline$-4 \mathrm{R} 4$ & do. & $4-05-92$ & $2,169.4$ & $9-09-92$ & $2,166.1$ & 3.3 \\
\hline$-5 \mathrm{~A} 4$ & do. & $3-31-92$ & $2,162.8$ & $9-09-92$ & $2,160.2$ & 2.6 \\
\hline$-30 \mathrm{R} 1^{1}$ & do. & $4-14-92$ & $2,217.4$ & -- & -- & $--^{2}$ \\
\hline $9 \mathrm{~N} / 8 \mathrm{~W}-6 \mathrm{~J} 1$ & do. & $3-31-92$ & $2,190.0$ & $9-09-92$ & $2,190.0$ & 0 \\
\hline 9N/9W-9A2 & do. & $4-05-92$ & $2,189.3$ & $9-09-92$ & $2,187.2$ & 2.1 \\
\hline$-10 \mathrm{R} 1$ & do. & 4-05-92 & $2,186.7$ & $9-09-92$ & $2,186.0$ & .7 \\
\hline$-13 N 1$ & do. & $3-13-92$ & $2,188.6$ & $9-10-92$ & $2,187.9$ & .7 \\
\hline$-15 J 1$ & do. & $4-07-92$ & $2,185.4$ & $9-10-92$ & $2,184.7$ & .7 \\
\hline$-18 \mathrm{Cl}$ & do. & $4-05-92$ & $2,179.0$ & $9-09-92$ & $2,177.8$ & 1.2 \\
\hline$-27 \mathrm{H} 2$ & do. & $4-05-92$ & $2,186.3$ & $9-09-92$ & $2,185.6$ & .7 \\
\hline$-28 \mathrm{~A} 4$ & do. & 4-05-92 & $2,183.7$ & $9-09-92$ & $2,182.1$ & 1.6 \\
\hline $9 \mathrm{~N} / 10 \mathrm{~W}-24 \mathrm{C} 1$ & Lancaster & $4-05-92$ & $2,167.1$ & $9-09-92$ & $2,164.4$ & 2.7 \\
\hline$-24 \mathrm{E} 1$ & do. & $4-05-92$ & $2,160.3$ & $9-09-92$ & $2,143.5^{3}$ & 16.8 \\
\hline$-25 \mathrm{P} 1$ & do. & $4-07-92$ & $2,163.9$ & $9-09-92$ & $2,154.4$ & 9.5 \\
\hline$-27 \mathrm{P} 3$ & do. & $--^{4}$ & $-{ }^{4}$ & $9-07-92$ & $2,157.0$ & $--^{2}$ \\
\hline$-28 \mathrm{~F} 2$ & do. & $4-04-92$ & $2,203.3$ & $9-07-92$ & $2,203.1$ & .2 \\
\hline$-28 \mathrm{H} 4$ & do. & $--^{4}$ & $--^{4}$ & $9-07-92$ & $2,156.7$ & $--^{2}$ \\
\hline$-34 \mathrm{R} 4$ & do. & $4-05-92$ & $2,159.6$ & $9-07-92$ & $2,153.5$ & 6.1 \\
\hline$-36 \mathrm{~F} 1$ & do. & $4-07-92$ & $2,163.3$ & $9-09-92$ & $2,152.1$ & 11.2 \\
\hline$-36 \mathrm{~J} 2$ & do. & $4-07-92$ & $2,163.6$ & $9-09-92$ & $2,151.2$ & 12.4 \\
\hline$-36 \mathrm{P} 1$ & do. & $4-07-92$ & $2,162.8$ & $9-09-92$ & $-{ }^{5}$ & $--^{2}$ \\
\hline$-36 \mathrm{P} 2$ & do. & 4-07-92 & $2,163.4$ & $9-09-92$ & $2,149.1$ & 14.3 \\
\hline $9 \mathrm{~N} / 11 \mathrm{~W}-36 \mathrm{Ll}$ & do. & $4-06-92$ & $2,197.0$ & $9-07-92$ & $2,189.2$ & 7.8 \\
\hline $9 \mathrm{~N} / 12 \mathrm{~W}-23 \mathrm{~N} 1$ & do. & $4-06-92$ & $2,219.8$ & $9-09-92$ & $2,219.5$ & .3 \\
\hline $10 \mathrm{~N} / 9 \mathrm{~W}-4 \mathrm{D} 1$ & North Muroc & $4-05-92$ & $2,177.0$ & $9-10-92$ & $2,174.7$ & 2.3 \\
\hline$-10 \mathrm{~B} 1$ & do. & $4-05-92$ & $2,182.8$ & $9-10-93$ & $2,182.5$ & .3 \\
\hline$-24 \mathrm{~A} 2$ & do. & $4-05-92$ & $2,196.0$ & $9-10-92$ & $2,195.6$ & .4 \\
\hline$-27 \mathrm{C} 2$ & Div. & $4-05-92$ & $2,193.3$ & $9-09-92$ & $2,193.0$ & .3 \\
\hline $11 N / 9 W-32 Q 1$ & North Muroc & 4-05-92 & $2,175.2$ & $9-10-92$ & $2,171.9$ & 3.3 \\
\hline$-36 \mathrm{R} 1$ & do. & 4-04-92 & $2,189.9$ & $9-10-92$ & $2,189.8$ & .1 \\
\hline
\end{tabular}

Footnotes at end of table. 
Table 5. Hydraulic heads and change in hydraulic heads for selected wells and piezometers used to plot the potentiometric surfaces of the aquifer system at Edwards Air Force Base, California, 1992--Continued

\begin{tabular}{|c|c|c|c|c|c|c|}
\hline \multirow{2}{*}{ State well No. } & \multirow{2}{*}{ Subbasin } & \multicolumn{2}{|c|}{ Hydraulic head, spring } & \multicolumn{2}{|c|}{ Hydraulic head, late summer } & \multirow{2}{*}{$\begin{array}{c}\text { Change in } \\
\text { hydraulic head } \\
\text { (ft) }\end{array}$} \\
\hline & & Date & Head & Date & Head & \\
\hline \multicolumn{7}{|c|}{ Completed in principal aquifer } \\
\hline $8 \mathrm{~N} / 10 \mathrm{~W}-18 \mathrm{P} 3$ & Lancaster & $3-31-92$ & $2,229.1$ & $9-09-92$ & $2,228.9$ & 0.2 \\
\hline$-28 \mathrm{~B} 1^{1}$ & do. & $4-15-92$ & $2,206.9$ & -- & -- & $--^{2}$ \\
\hline $8 \mathrm{~N} / 11 \mathrm{~W}-14 \mathrm{R} 1$ & do. & 4-04-92 & $2,227.4$ & $9-09-92$ & $2,227.1$ & .3 \\
\hline$-15 Q 1$ & do. & 4-04-92 & $2,225.9$ & $9-09-92$ & $2,225.7$ & .2 \\
\hline$-22 \mathrm{P} 2^{1}$ & do. & 4-14-92 & $2,223.2$ & -- & -- & $--^{2}$ \\
\hline$-24 R 2^{1}$ & do. & 4-14-92 & $2,227.4$ & -- & - & $--^{2}$ \\
\hline$-34 D 2^{1}$ & do. & $4-15-92$ & $2,217.4$ & -- & -- & $--^{2}$ \\
\hline$-34 R 2^{1}$ & do. & $4-15-92$ & $2,226.8$ & -- & -- & $--^{2}$ \\
\hline $8 \mathrm{~N} / 12 \mathrm{~W}-2 \mathrm{Q} 1$ & do. & 4-06-92 & $2,234.8$ & $9-09-92$ & $2,232.0$ & 2.8 \\
\hline$-10 \mathrm{~J} 1$ & do. & 4-06-92 & $2,252.5$ & $9-09-92$ & $2,251.9$ & .6 \\
\hline$-26 \mathrm{~F} 1^{1}$ & do. & 4-13-92 & $2,279.5$ & -- & -- & $--^{2}$ \\
\hline$-28 D 1^{1}$ & do. & 4-13-92 & $2,248.9$ & -- & -- & $--^{2}$ \\
\hline$-34 \mathrm{~K} 1^{1}$ & do. & 4-13-92 & $2,259.5$ & -- & -- & $--^{2}$ \\
\hline $9 \mathrm{~N} / 12 \mathrm{~W}-33 \mathrm{P} 1^{1}$ & do. & 4-16-92 & $2,235.4$ & -- & -- & $--^{2}$ \\
\hline \multicolumn{7}{|c|}{ Completed in the unconfined aquifer } \\
\hline $9 \mathrm{~N} / 10 \mathrm{~W}-8 \mathrm{P} 1$ & Unnamed & $4-05-92$ & $2,289.0$ & $9-08-92$ & $2,289.0$ & 0 \\
\hline$-16 \mathrm{~F} 1$ & do. & $4-05-92$ & $2,209.4$ & $9-07-92$ & $2,209.7$ & -.3 \\
\hline$-16 L 1$ & do. & $4-05-92$ & $2,203.2$ & $9-08-92$ & $2,201.8$ & 1.4 \\
\hline$-16 \mathrm{~L} 2$ & do. & $4-05-92$ & $2,206.8$ & $9-07-92$ & $2,205.9$ & .9 \\
\hline$-16 \mathrm{~L} 3$ & do. & $4-05-92$ & $2,205.9$ & $9-07-92$ & $2,206.1$ & -.2 \\
\hline$-16 \mathrm{M} 1$ & do. & $4-05-92$ & $2,203.7$ & $9-07-92$ & $2,203.4$ & .3 \\
\hline$-16 \mathrm{~N} 1$ & do. & 4-05-92 & $2,203.7$ & $9-07-92$ & $2,203.5$ & .2 \\
\hline$-16 \mathrm{P} 1$ & do. & $4-05-92$ & $2,203.3$ & $9-07-92$ & $2,202.1$ & 1.2 \\
\hline$-16 R 3$ & do. & $3-30-92$ & $2,211.0$ & $9-07-92$ & $2,208.3$ & 2.7 \\
\hline$-16 R 4$ & do. & $4-05-92$ & $2,208.5$ & $10-06-92$ & $2,205.6$ & 2.9 \\
\hline
\end{tabular}

${ }^{1}$ Wells monitored annually for Antelope Valley-East KErn Water Agency (Johnson and Fong-Frydendal, 1993).

${ }^{2}$ Not able to calculate.

${ }^{3}$ Influenced by pumping from well $9 \mathrm{~N} / 10 \mathrm{~W}-24 \mathrm{E} 2$.

${ }^{4}$ Drilled in August 1992.

${ }^{5}$ Well pumping. 
across Rogers Lake did not measurably change (fig. 19).

In the Graham Ranch well field, hydraulic heads in production wells 9N/10W-16P1 and -16R4 declined 1 and $3 \mathrm{ft}$, respectively, from spring to late summer (table 5, fig. 19). The north-south, 2,210-foot potentiometric contours that defined the ground-water divide in the spring (fig. 15) merged and were plotted northeast of the well field in late summer (fig. 16).

\section{Principal Aquifer}

The potentiometric surface of the principal aquifer near wells 8N/10W-18P3 and 8N/11W-14R1 and $-15 Q 1$ was relatively flat (figs. 17 and 18), whereas the slope of the potentiometric surface steepened toward a regional ground-water depression south of Redman (Londquist and others, 1993, fig. 5). Heads in wells 8N/10W-18P3 and 8N/11W-14R1 and -15Q1 changed less than $0.5 \mathrm{ft}$ between April and September (table 5). The potentiometric-surface contours of the principal aquifer southwest of Rosamond Lake ranged from about 2,220 to $2,280 \mathrm{ft}$ above sea level (figs. 17 and 18). Because ground-water levels for wells south of the base were not available, the potentiometric surface of the principal aquifer for late summer is inferred.

\section{Ground-Water Flow}

Ground water flows from areas of high hydraulic head to areas of low hydraulic head. Flow may be vertical as well as horizontal. Hydraulic gradient is the ratio of the difference in hydraulic head between two wells and the distance between the wells. Vertical hydraulic gradient is the ratio of the difference in head in nested or clustered wells and the difference in altitude of the midpoint of the screened interval.

Four subregional ground-water-flow directions were identified in the deep aquifer: (1) north and northeast from the Lancaster subbasin to the Branch Park and South Track well fields; (2) south and southwest from the central part of Rogers Lake to the South Base and South Track well fields; (3) west from the alluvial fan upslope (east) of the Phillips Laboratory well fields to Rogers Lake; and (4) north from a ground-water divide in the north-central part of Rogers Lake to the North Base well field (figs. 15 and 16). The spring and late summer hydraulic gradients for these four flow directions are listed in table 6.
Table 6. Hydraulic gradients for four subregional ground-water-flow directions in the deep aquifer at Edwards Air Force Base, California, 1992

\begin{tabular}{lrc}
\hline \multirow{2}{*}{ Flow direction } & \multicolumn{2}{c}{$\begin{array}{c}\text { Hydraulic gradients, } \\
\text { in feet }\end{array}$} \\
\cline { 2 - 3 } & Spring & Late summer \\
\hline 8N/10W-4R4 to 9N/10W-34R4 & 0.0012 & 0.0016 \\
9N/9W-28A4 to 9N/10W-36J2 & .0011 & .0017 \\
9N/9W-13N1 to 9N/9W-15J1 & .0005 & .0005 \\
10N/9W-10B1 to 10N/9W-4D1 & .0006 & .0008 \\
\hline
\end{tabular}

Hydraulic heads in the piezometers completed in the deep aquifer (figs. $6 A, 6 B, 6 C, 6 F, 6 G, 6 J$, and $6 K$ ) indicate that the vertical gradient generally is downward from the upper confined zone to the lower confined zone. During the summer pumping season, heads in piezometers near the South Track well field (figs. $6 C, 6 F$, and $6 G$ ) indicate that the vertical gradient had reversed at these sites causing upward flow from the lower confined zone to the upper confined zone. This may cause hard, saline type water (Londquist and others, 1993) to move upward from the lower confined zone to the upper confined zone.

\section{EXPLANATION FOR FIGURE 19}

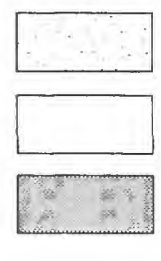

\section{PLAYA SURFACE}

\section{ALLUVIUM}

\section{BEDROCK}

\section{......... GROUND-WATER DIVIDE \\ - $S$ - STRUCTURAL BOUNDARY \\ — - EDWARDS AIR FORCE BASE BOUNDARY \\ - 4 - LINE OF EQUAL HEAD DECLINE-Spring to late summer 1992. Interval 1 foot. \\ Dashed where approximate \\ 24E1 、 WELL OR PIEZOMETER AND NUMBER- With hydraulic head changes for the deep aquifer \\ ${ }^{30 R 1}$ WELL OR PIEZOMETER AND NUMBER- Not monitored for this study}




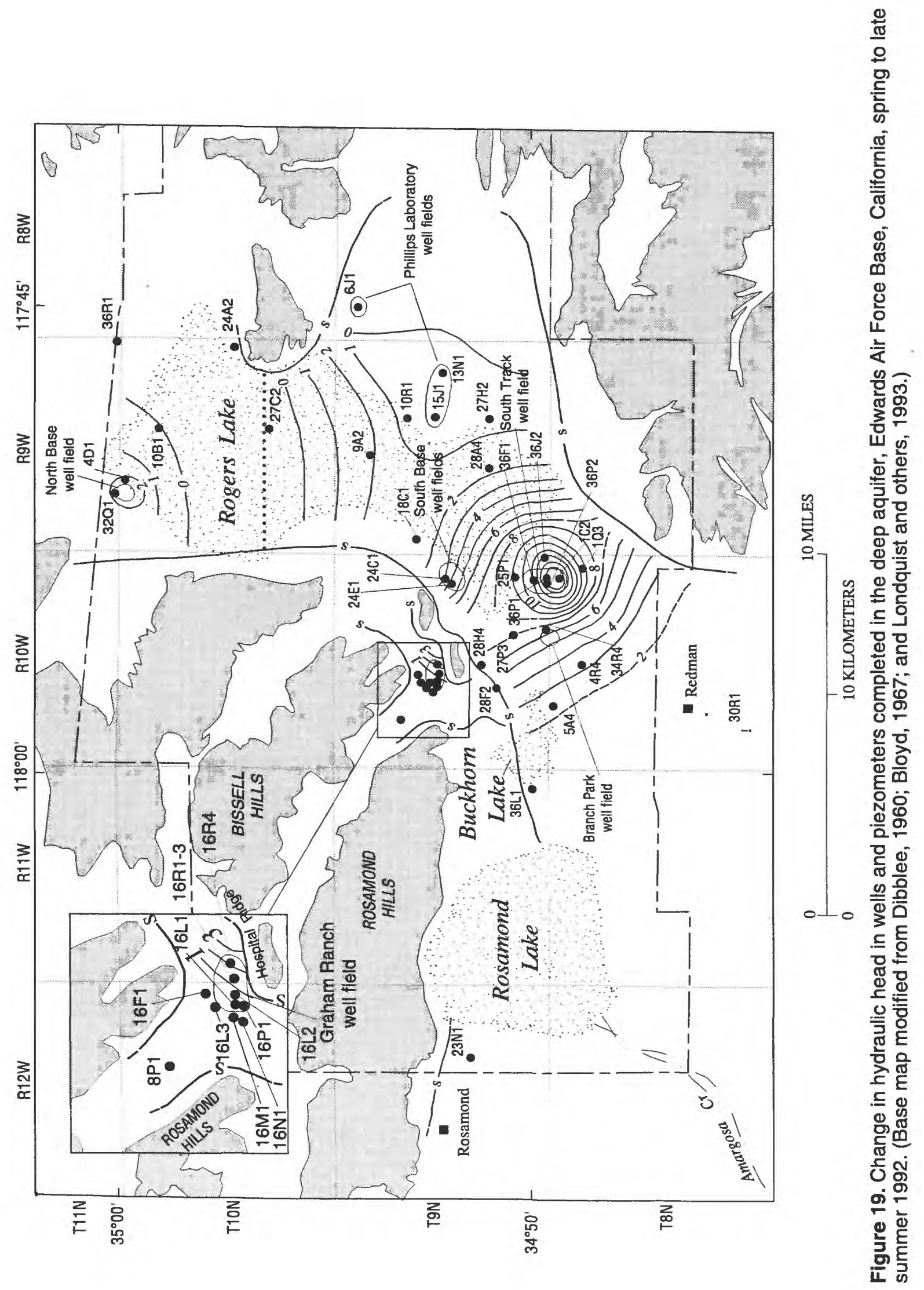




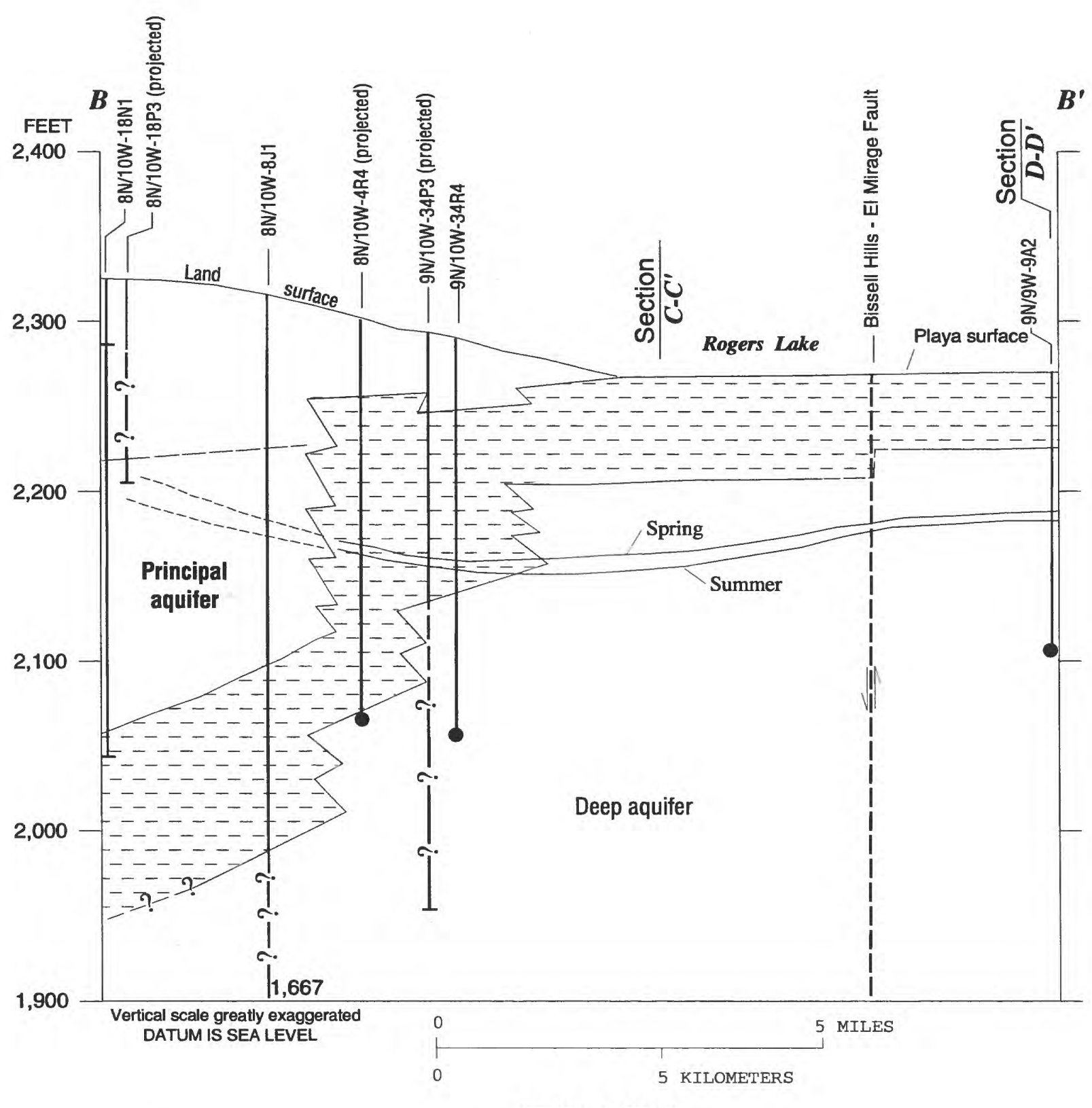

EXPLANATION

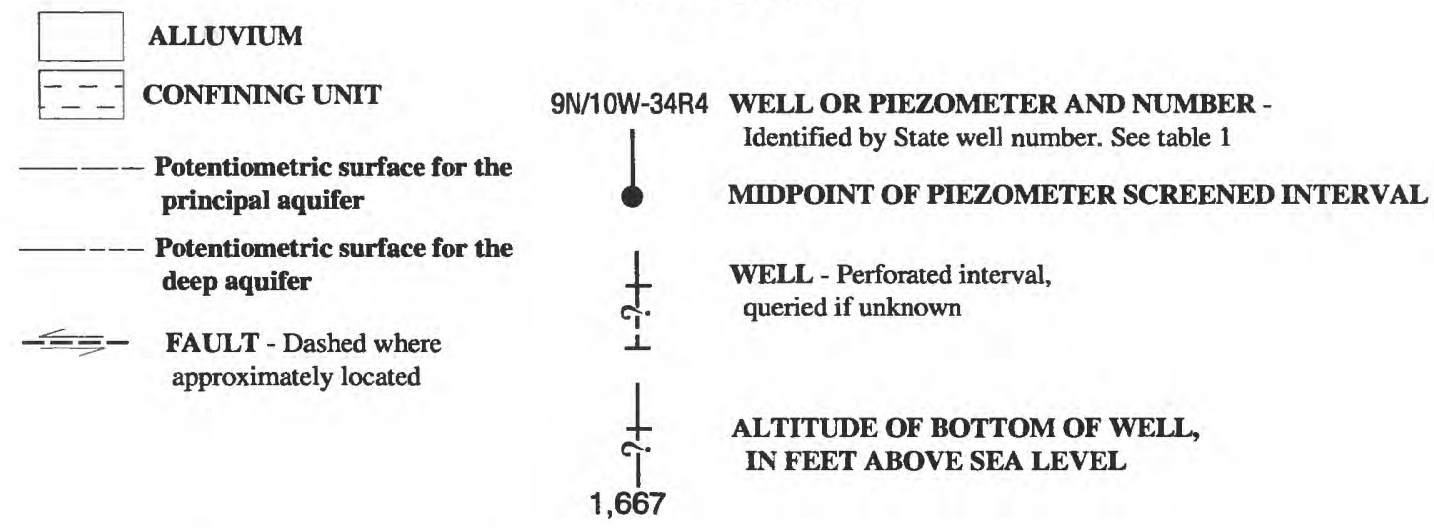

Figure 20. Hydraulic-head profiles for geologic sections $B-B^{\prime}, C-C^{\prime}$ and $D-D$, Edwards Air Force Base, California. 


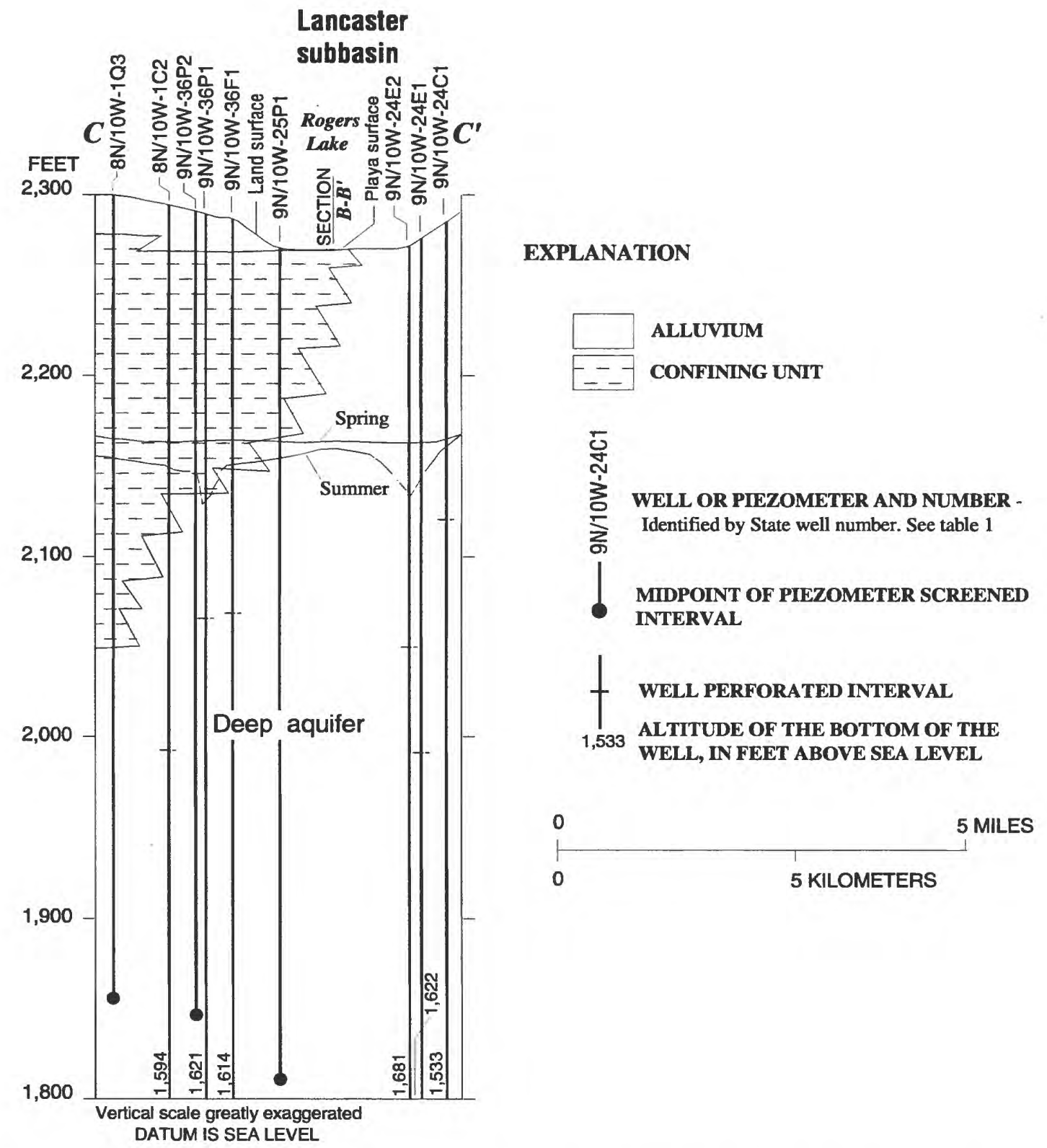

Figure 20. Hydraulic-head profiles for geologic sections $B-B, C-C$ and $D-D$, Edwards Air Force Base, California--Continued. 

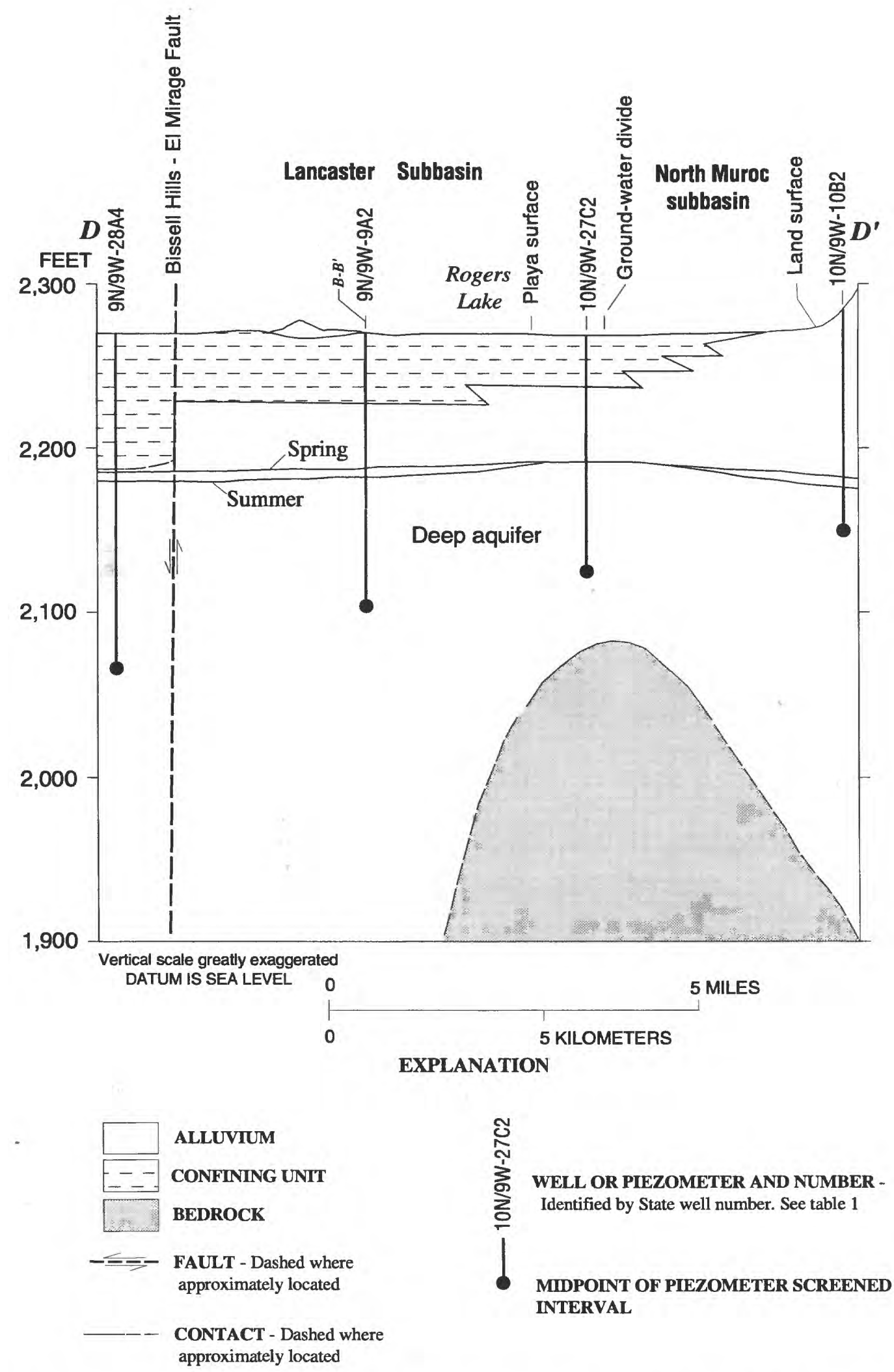

Figure 20. Hydraulic-head profiles for geologic sections $B-B^{\prime}, C-C$ and $D-D$, Edwards Air Force Base, California--Continued. 
Johnson (1911) reported that alkali deposition on the playa surface of Rogers Lake possibly was due to ground-water evaporation. Historical evidence of artesian flow in wells completed in the deep aquifer in this area indicated an upward vertical gradient from the deep aquifer through the confining unit (Johnson, 1911). According to historical records for the late 1950's, well 8N/9W-6D1, completed in the confining unit, and wells 9N/9W-27H1 and 9N/10W-24C1, completed in the deep aquifer, had similar water levelsabout 20 to $25 \mathrm{ft}$ below land surface (Londquist and others, 1993)-indicating equilibrium between heads in the deep aquifer and heads in the confining unit. In 1992, hydraulic heads in the piezometers completed in the confining unit were higher than those completed in the confined aquifer, indicating that the vertical gradient is now downward from the confining unit to the deep aquifer. Because the vertical gradient between the confining unit and deep aquifer is downward, the confining unit is being dewatered. This dewatering is causing compaction of fine-grained sediments, which, in turn, results in land-surface deformation.

Hydraulic heads in the confining unit south of the principal-aquifer boundary are not known. Further study in this area is needed to determine the vertical gradients between the deep aquifer and the confining unit and between the confining unit and the principal aquifer.

In the area of the Graham Ranch well field, ground water flows toward the pumping centers of production wells 9N/10W-16P1 and -16R4 (figs. 15 and 16). Higher hydraulic heads in the deepest piezometer, 9N/10W-16R1 (fig. 6N), and lower hydraulic head in the shallowest piezometer, 9N/10W-16R3, indicate that the vertical hydraulic gradient is upward. Near well 9N/12W-23N1, ground water probably flows westward away from Rosamond Lake (figs. 15 and 16).

In the principal aquifer, ground water flows south and southeastward, away from EAFB and radially from the ground-water mound identified southwest of Rosamond Lake (figs. 17 and 18). The groundwater mound is at the terminus of Amargosa Creek (figs. 17 and 18) where the Los Angeles Sanitation District maintains lagoons that contain treated wastewater that is discharged from their sanitation facilities west of Sierra Highway (fig. 1). This ground-water mound may indicate that surface-water runoff and treated wastewater recharges the principal aquifer at that location. Spring water levels in wells $8 \mathrm{~N} / 12 \mathrm{~W}-$ $2 \mathrm{Q} 1,-10 \mathrm{~J} 1,-26 \mathrm{~F} 1,-28 \mathrm{D} 1$, and $-34 \mathrm{~K} 1$ indicate ground water flows south and westward from this mound. Water levels in wells $8 \mathrm{~N} / 12 \mathrm{~W}-2 \mathrm{Q} 1$ and -10J1 declined less than $2 \mathrm{ft}$ from April to September (table 5, fig. 14), indicating that the principal aquifer may respond to seasonal recharge fluctuations and increased pumping west and southwest of Rosamond during the summer months. Public and private supply wells in this area, which may be screened above and below the confining unit, could affect heads and ground-water flow in both the principal and deep aquifers.

\section{SUMMARY AND CONCLUSIONS}

A ground-water-level monitoring program was implemented at Edwards Air Force Base (EAFB), Antelope Valley, California, to monitor spatial and temporal changes in the potentiometric surfaces of the aquifer system that are affected by ground-water pumping. Potentiometric-surface maps are needed to determine the correlation between declining groundwater levels and the distribution of land subsidence. The ground-water-level monitoring program focused on areas of EAFB where ground-water pumping occurs, especially near Rogers Lake. Well-construction, historical water-level, and lithologic data were compiled for 118 wells and piezometers on and near the base, and monthly measurements of ground-water levels were made for 82 wells and piezometers on the base from January to December 1992.

The ground-water-level monitoring program involved three phases of data collection: (1) well canvassing and selection, (2) geodetic surveying to determine vertical datum for each well, and (3) monthly measurements of ground-water levels. Selection of wells used in this monitoring program was based on (1) measurable ground-water levels, (2) accessibility of the wells, (3) proximity to the EAFB well fields and Rogers Lake, (4) proximity to other suitable wells to avoid redundancy, and (5) the position of the screened or perforated interval in the well.

Ground-water levels generally ranged from about 95 to $130 \mathrm{ft}$ below land surface in wells and piezometers in the North Muroc subbasin, 70 to 200 feet below land surface in the deep aquifer in the Lancaster subbasin, 35 to 95 feet below land surface in the principal aquifer in the Lancaster subbasin, and 100 to 125 feet below land surface in or near the Graham Ranch well field. Total hydraulic heads, or heads, were computed using these ground-water levels and land-surface altitudes. Heads generally ranged from about 2,170 to 2,195 feet above sea level in the North Muroc subbasin, 2,150 to 2,200 feet above sea level in the 
deep aquifer in the Lancaster subbasin, 2,225 to 2,250 feet above sea level in the principal aquifer in the Lancaster subbasin, and 2,200 to 2,215 feet above sea level in the Graham Ranch well field. Heads in wells and piezometers completed in the confining unit ranged from about 2,210 to 2,275 feet above sea level.

Heads for the piezometers completed in the deep aquifer, west, south, east, and in the South Track well field, which were higher than the lower contact of the confining unit, indicate confined, nonflowing, artesian conditions. Heads in piezometers completed in the deep aquifer north of the South Track well field which were lower than the lower contact of the confining unit, indicate locally unconfined aquifer conditions.

Heads in the piezometers completed below 1,500 feet above sea level indicate a delay in response to seasonal recharge and discharge stresses in the aquifer, which, in turn, indicates a poor hydraulic connection between the upper and lower confined zones. This poor hydraulic connection probably is due to the consolidation of the deeper alluvium.

Total pumpage for 1992 from seven well fields on EAFB was about 1,700 million gallons or 5,225 acre-feet. Total pumpage of about 697.3 million gallons, 2,140 acre-feet, from the EAFB production wells in the South Track well field caused heads to decline about 9 to 10 feet. Drawdowns in these wells ranged from about 20 to 30 feet. About 397.2 million gallons, 1,219 acre-feet, was pumped from the North Base well field, lowering heads about 2 to 3 feet. About 379.7 million gallons, 1,165 acre-feet, was pumped from the South Base well field. Large drawdowns of 50 to 130 feet in the South Base wells may indicate low transmissivity, possibly a result of dewatering and compaction of the fine-grained layers.

Boundaries of the aquifer system were determined using surface and borehole geophysical data, lithologic logs, and ground-water-level data. Three types of no-flow boundaries were identified: structural boundaries, a principal-aquifer boundary, and groundwater divides.

Structural boundaries to the south and southeast of Rogers Lake and north of the Phillips Laboratory well field are bedrock-alluvium contacts. Another structural boundary south of the Rosamond and Bissell Hills, striking southwest-northeast from the eastern shore of Rosamond Lake to Buckhorn Lake, is a permeable/less permeable alluvium contact that coincides with the northwestern boundary of the Antelope Valley Fault Zone. The boundary extends across Buckhorn Lake, becomes a bedrock-alluvium boundary along the southeastern edge of Hospital Ridge, then strikes northward, crosses the buried Bissell Hills-El Mirage Fault, and parallels exposed bedrock west of Rogers Lake. The boundary probably continues northward west of the North Base well field, but, because of insufficient data, its position is not known.
Ground-water-level and lithologic data were used to determine the position of the principal-aquifer boundary. The confining unit is at or near land surface in wells or piezometers north of the boundary. South of the boundary, wells generally are completed in the principal aquifer; the deep aquifer is confined. The lateral, northeastern extent of the confining unit is in the south-central part of Rogers Lake. North of the South Track well field, the deep aquifer is unconfined. A ground-water divide strikes east-west across the northcentral part of Rogers Lake. The divide separates the Lancaster and North Muroc subbasins and prevents ground water from flowing between the two subbasins. The boundaries of the unconfined aquifer in the Graham Ranch well field are irregularly shaped bedrock-alluvium contacts. A ground-water divide separates the EAFB production wells in this small subbasin.

Hydraulic heads of base production wells, abandoned wells, and one piezometer from each of the USGS piezometer sites were used to contour seasonal potentiometric surfaces of the aquifer system at EAFB. Mapping of the potentiometric surfaces was done to provide a visual interpretation of the areal extent and generalized ground-water-flow paths of the aquifer system. Changes in the potentiometric surfaces of the aquifer system at EAFB were relatively small, with heads ranging from about 2,160 to 2,220 feet above sea level in the deep aquifer and about 2,200 to 2,280 feet above sea level in the principal aquifer in the Lancaster subbasin; about 2,180 to 2,190 feet above sea level in the North Muroc subbasin; and about 2,210 to 2,290 feet in the Graham Ranch wellfield area.

The potentiometric surface of the deep aquifer for spring 1992 ranged from 2,160 to 2,180 feet above sea level forming a regional ground-water depression in the areas between, and adjacent to, the South Track, South Base, and Branch Park well fields. By late summer, the potentiometric surface had declined about 10 feet in the South Track well field, about 3 feet in the South Base well field, and about 8 feet in the Branch Park well field. A 10-foot decline in the potentiometric surface and 20- to 30-foot drawdowns in the EAFB production wells caused local, unconfined conditions in the deep aquifer in the South Track well field. The potentiometric surfaces near the Phillips Laboratory and North Base well fields declined about 0 to 3 feet between spring and late summer. The potentiometric surface near the ground-water divide across Rogers Lake did not change measurably. The potentiometric surface in and near the Graham Ranch well field declined 1 to 3 feet.

The potentiometric surface of the principal aquifer near wells along the southern boundary of the base were relatively flat, whereas the slope of the potentio-metric surface steepened toward a regional ground-water depression south of Redman. The potentiometric-surface contours of the principal 
aquifer southwest of Rosamond Lake ranged from about 2,220 to 2,280 feet above sea level, forming a ground-water mound beneath the terminus of Amargosa Creek where surface-water runoff and treated wastewater discharge probably recharge the principal aquifer. The principal aquifer in this area may respond to both seasonal recharge fluctuations and increased pumping in the Rosamond area during the summer months. The configuration of the potentiometric surface of the deep aquifer south and west of EAFB is unknown.

Four major ground-water-flow directions were identified in the deep aquifer: (1) north and northeast from the Lancaster subbasin to the Branch Park and South Track well fields; (2) south and southwest from the central part of Rogers Lake toward the South Base and South Track well fields; (3) west from the Phillips Laboratory well fields to Rogers Lake, and (4) north from a ground-water divide in the north-central part of Rogers Lake to the North Base well field. Groundwater flow in the area of the Graham Ranch well field is toward the EAFB production wells. Ground-water flow in the principal aquifer is south and southeastward away from EAFB and radially from the groundwater mound southwest of Rosamond Lake. Groundwater flow near well 9N/12W-23N1 probably is westward away from Rosamond Lake.

Vertical head differences in piezometers constructed in the Lancaster and North Muroc subbasins indicate that vertical ground-water flow generally is downward from the upper confined zone into the lower confined zone. In the summer months, increased pumping in the South Track well field caused flow to reverse in the deep aquifer and to move upward from the lower confined zone into the upper confined zone. Vertical head differences in piezometers in the Graham Ranch well field indicate flow is upward.

\section{References Cited}

Blodgett, J.C., and Williams, J.S., 1992, Land subsidence and problems affecting land use at Edwards Air Force Base and vicinity, California, 1990: U.S. Geological Survey Water-Resources Investigations Report 92 4035, $25 \mathrm{p}$.

Bloyd, R.M., Jr., 1967, Water resources of the Antelope Valley-East Kern Water Agency area, California: U.S. Geological Survey Open-File Report, 69 p.

Dibblee, T.W., Jr., 1960, Geology of Rogers Lake and Kramer quadrangles, California: U.S. Geological Survey Bulletin 1089-B, p. 73-139.

Duell, L.F.W., Jr., 1987, Geohydrology of the Antelope Valley area, California, and design for a ground-waterquality monitoring network: U.S. Geological Survey Water-Resources Investigations Report 84-4081, 72 p.

Durbin, T.J., 1978, Calibration of a mathematical model of the Antelope Valley ground-water basin, California: U.S. Geological Survey Water-Supply Paper 2046, $51 \mathrm{p}$.
Dutcher, L.C., Bader, J.S., Hiltgen, W.J., and others, 1962, Data on wells in the Edwards Air Force Base area, California: California Department of Water Resources Bulletin no. 91-6, 209 p.

Dutcher, L.C., and Worts, G.F., 1963, Geology, hydrology, and water supply of Edwards Air Force Base, Kern County, California: U.S. Geological Survey Open-File Report, 225 p.

Franke, O.L., Reilly, T.E., and Bennett, G.D., 1987, Definition of boundary and initial conditions in the analysis of saturated ground-water flow systems--An introduction: U.S. Geological Survey Techniques of WaterResources Investigations, book 3, chap. B5, 15 p.

Galloway, D.L., 1993, Coseismic volume strain associated with the Landers Earthquake: An analysis of aquifer fluid-pressure responses, Antelope Valley, California (abs.): EOS, Transactions of the American Geophysical Union, v. 74, no. 16, p. 317.

Johnson, H.R., 1911, Water resources of Antelope Valley, California: U.S. Geological Survey Water-Supply Paper 278, 92 p.

Johnson, J.A., and Fong-Frydenal, L.J., 1993, Water resources data--California, water year 1992. Volume 5. Ground-water data: U.S. Geological Survey WaterData Report CA-92-5, 437 p.

Londquist, C.J., Rewis, D.L., Galloway, D.L., and McCaffrey, W.F., 1993, Hydrogeology and land subsidence, Edwards Air Force Base, Antelope Valley, California, January 1989-December 1991: U.S. Geological Water-Resources Investigations Report 934114, 74 p.

Rewis, D.L., 1993, Drilling, construction, and subsurface data for piezometers on Edwards Air Force Base, Antelope Valley, California, 1991-1992: U.S. Geological Survey Open-File Report 93-148, 35 p.

Snyder, J.H., 1955, Ground water in California: The experience of Antelope Valley: Berkeley, University of California, Division of Agriculture, Giannini Foundation Ground-Water Studies no. 2, 171 p.

U.S. Army Corps of Engineers, 1961, Report on water well drilling, water well no. 3, Edwards Air Force Base: U.S. Army Corps of Engineers, Los Angeles District, $7 \mathrm{p}$.

U.S. Department of Commerce, 1966, Vertical control data, Quad 341181: 13 p.

Zhody, A.A., and Bisdorf, R.J., 1990, Ground-water exploration using deep Schlumberger soundings at Edwards Air Force Base, California, Part 1: Graham Ranch and Rogers Lake: U.S. Geological Survey Open-File Report 90-536, 95 p.

----1991, Ground-water exploration using deep Schlumberger soundings at Edwards Air Force Base, California. Part II. Rogers Lake and north Edwards Air Force Base: U.S. Geological Survey Open-File Report 91-446, 109 p. 


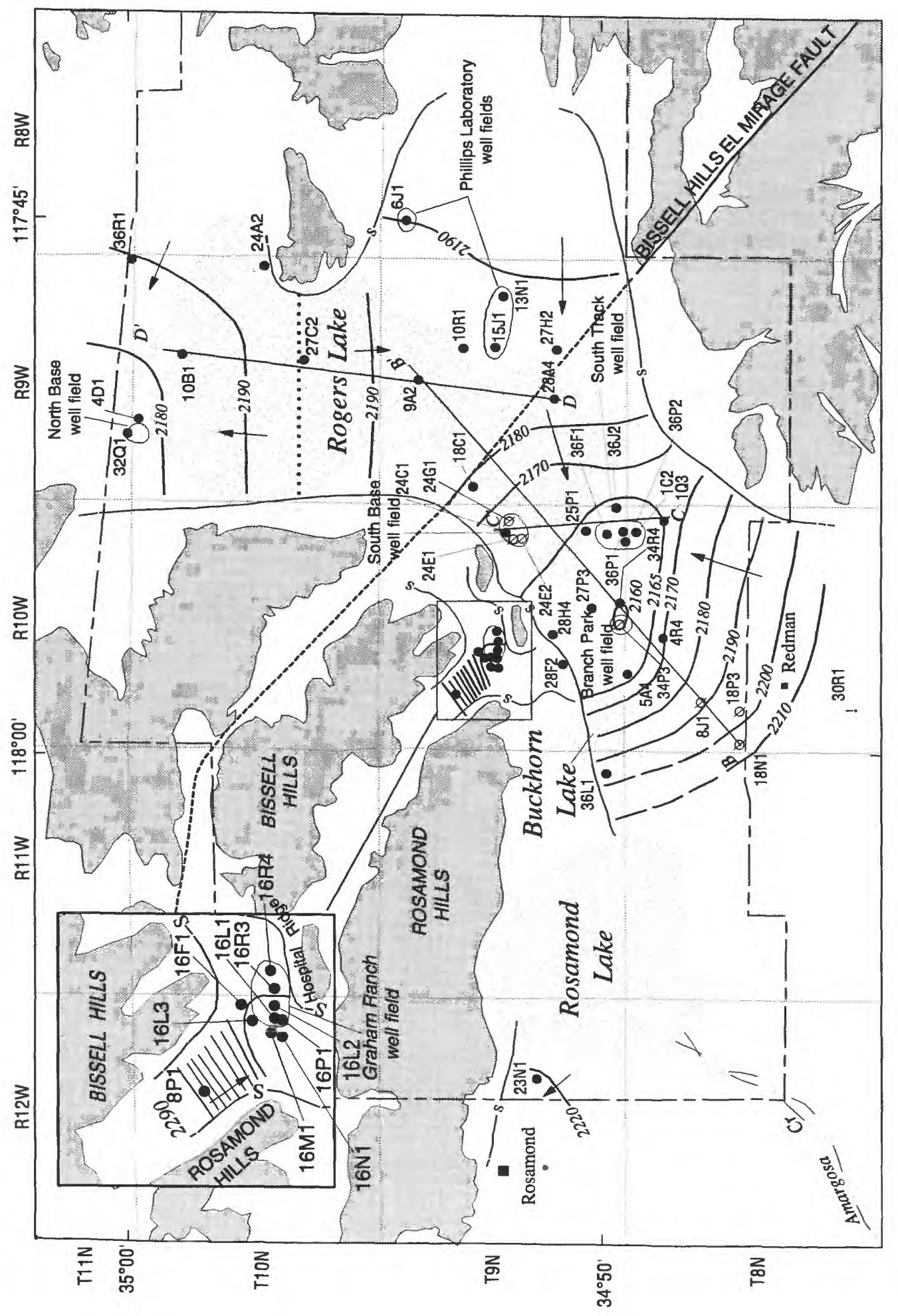

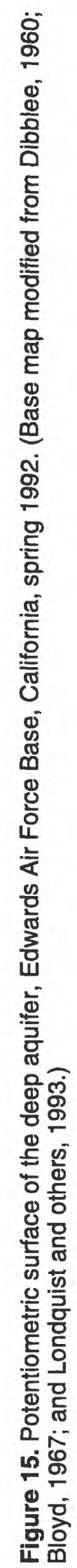




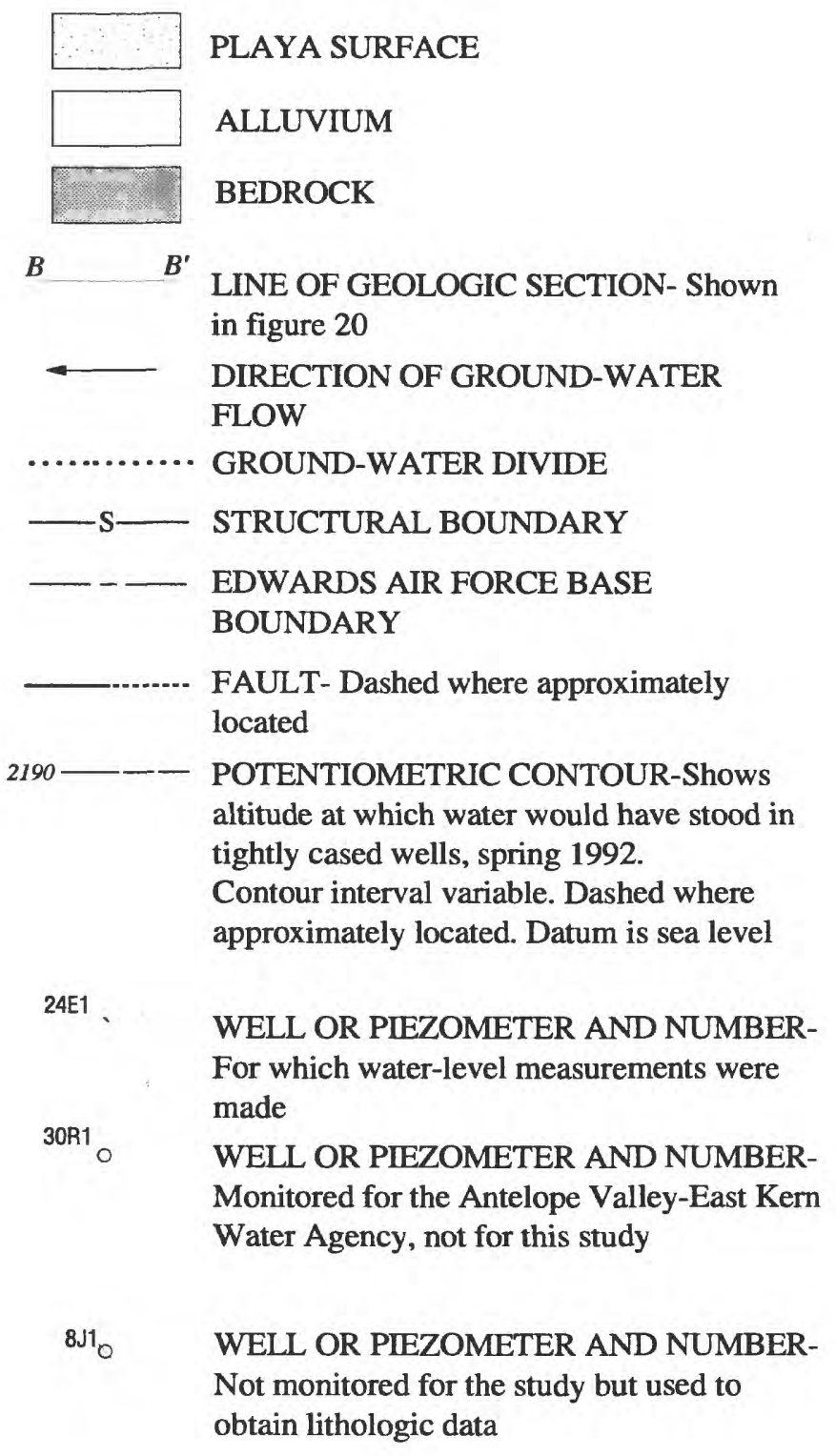




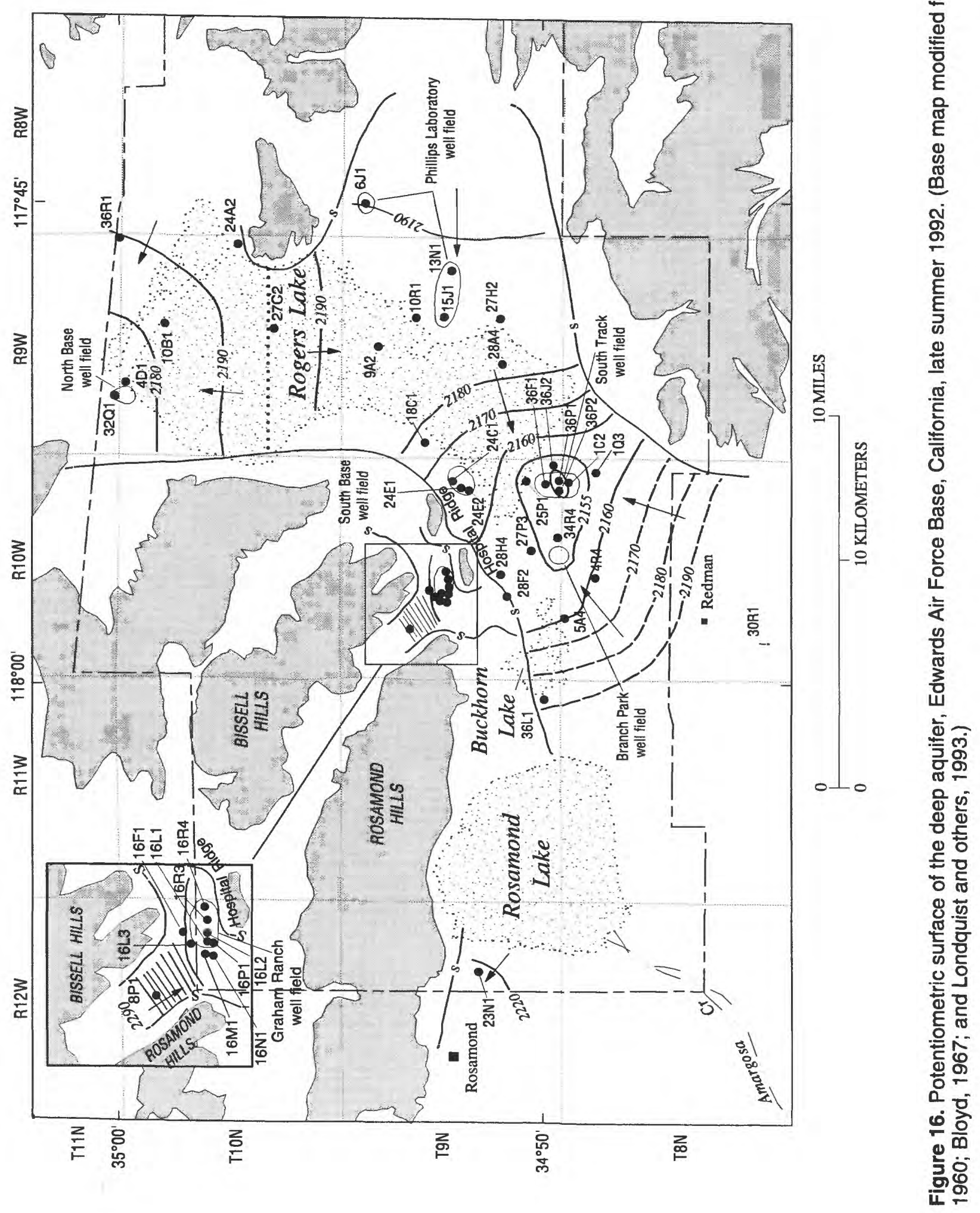




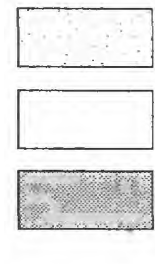

\section{PLAYA SURFACE}

ALLUVIUM

BEDROCK

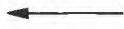

DIRECTION OF GROUND-WATER

FLOW

.......... GROUND-WATER DIVIDE

-S- STRUCTURAL BOUNDARY

- - EDWARDS AIR FORCE BASE BOUNDARY

2190 POTENTIOMETRIC CONTOUR-Shows altitude at which water would have stood in tightly cased wells, late summer 1992. Contour interval variable. Dashed where approximately located. Datum is sea level

24E1 、 WELL OR PIEZOMETER AND NUMBERFor which water-level measurements were made

30R1 WELL OR PIEZOMETER AND NUMBERMonitored for the Antelope Valley-East Kern Water Agency, not for this study 


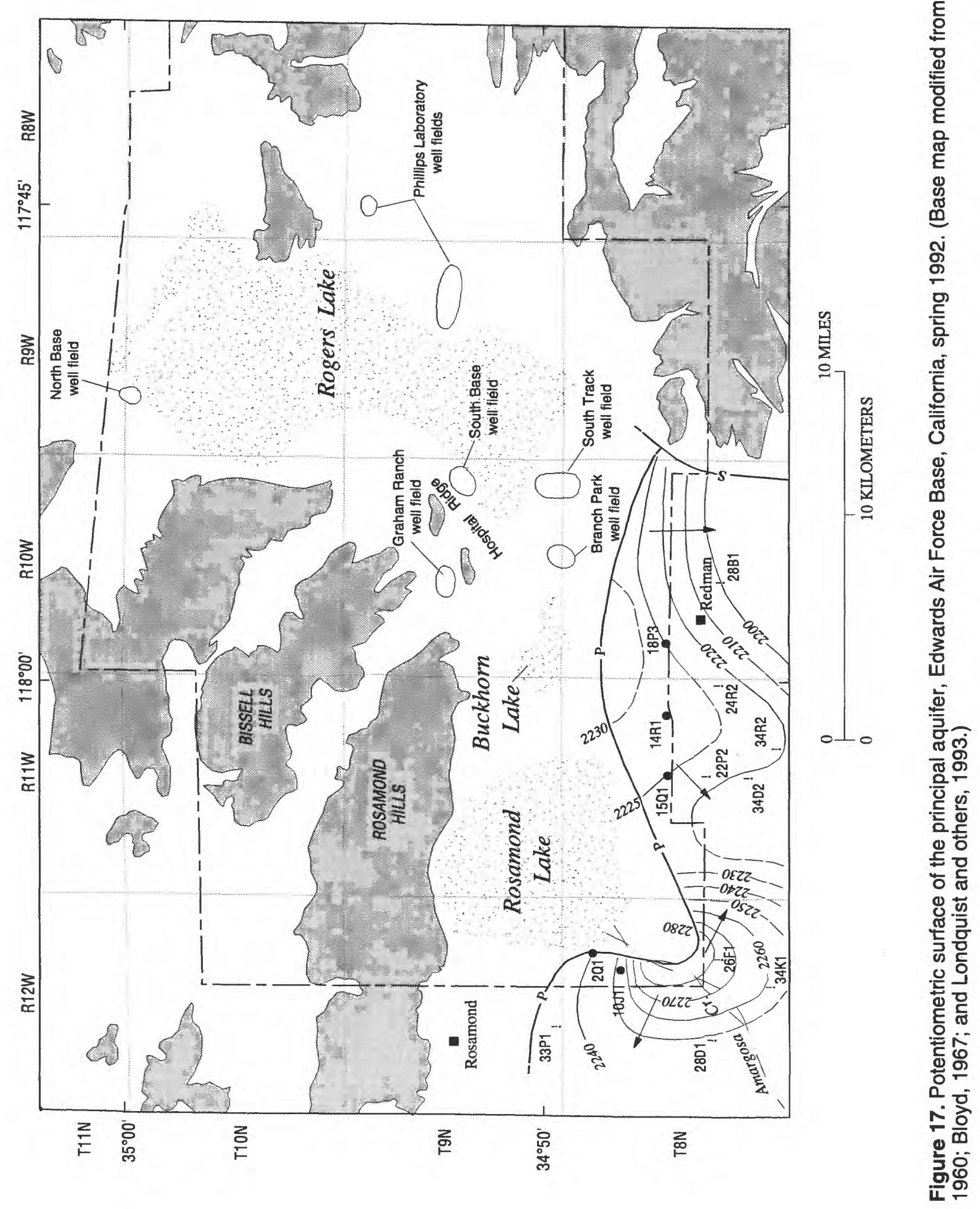


EXPLANATION FOR FIGURE 17

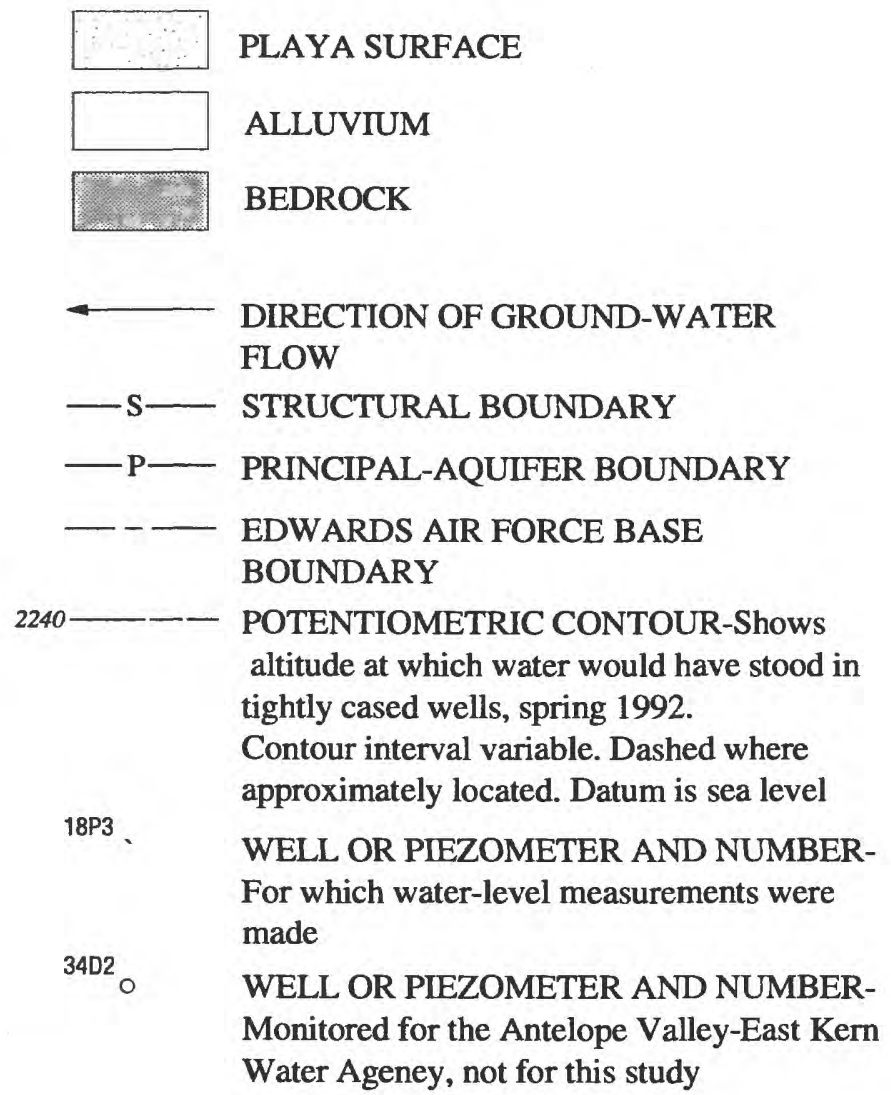




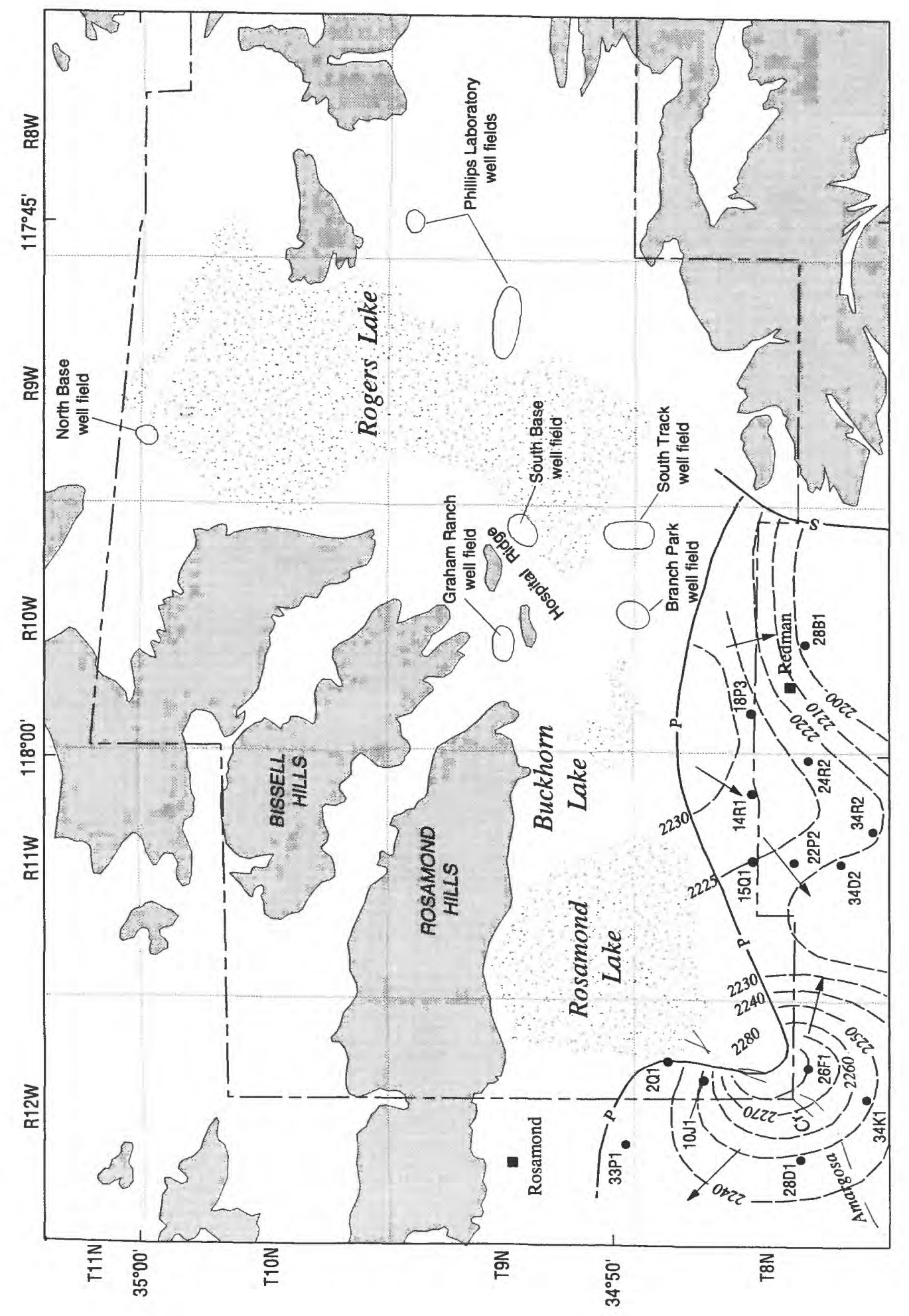

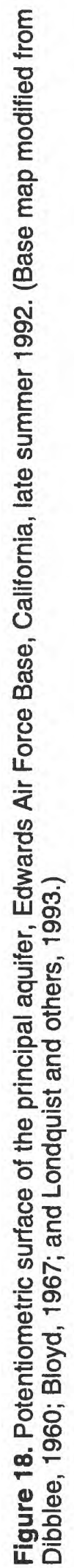




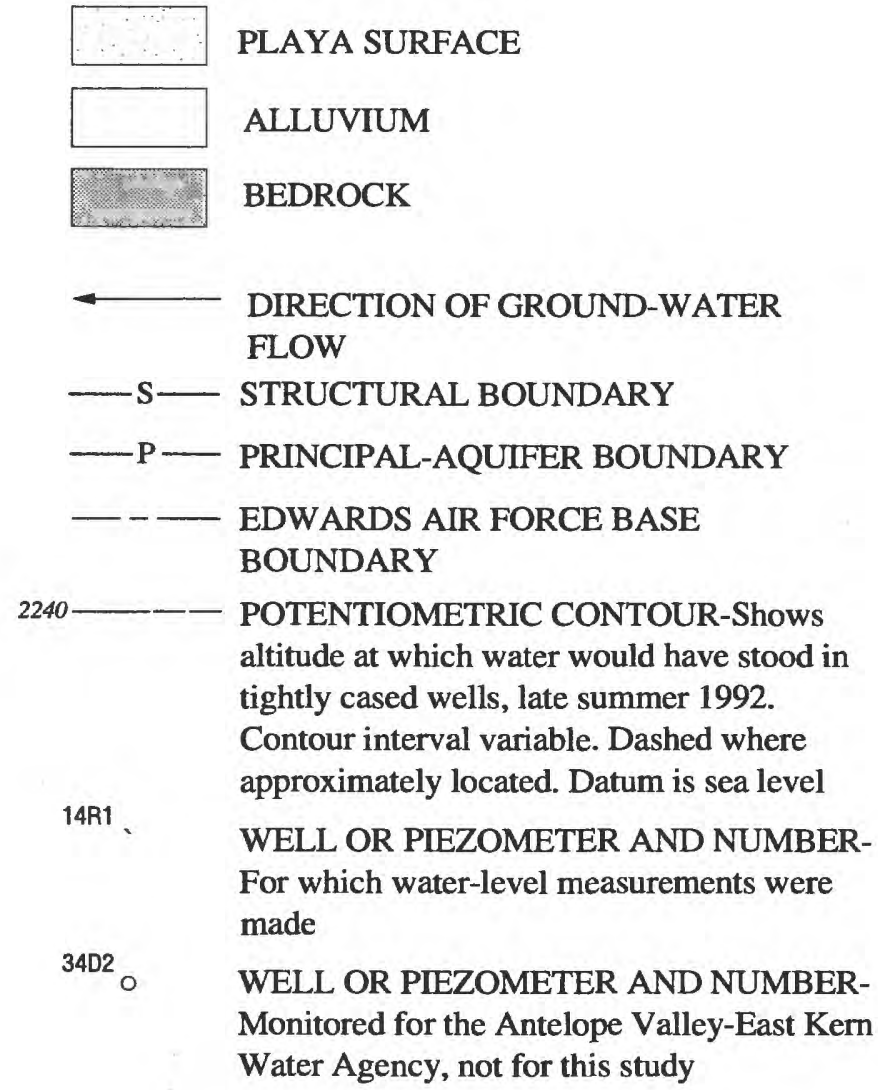

